\title{
TOPICS IN FUNCTIONAL ANALYSIS
}

\author{
A Dissertation \\ by \\ MARCH TIAN BOEDIHARDJO
}

\begin{abstract}
Submitted to the Office of Graduate and Professional Studies of Texas A\&M University in partial fulfillment of the requirements for the degree of DOCTOR OF PHILOSOPHY
\end{abstract}

$\begin{array}{ll}\begin{array}{ll}\text { Chair of Committee, } \\ \text { Co-Chair of Committee, }\end{array} & \text { William B. Johnson } \\ \text { Committee Members, } & \text { Anthony T. Cahill } \\ & \begin{array}{l}\text { Ronald G. Douglas } \\ \text { Ciprian Foias }\end{array} \\ & \text { Thomas Schlumprecht } \\ \text { Head of Department, } & \text { Emil Straube }\end{array}$

May 2016

Major Subject: Mathematics

Copyright 2016 March Tian Boedihardjo 


\begin{abstract}
I give a geometric characterization of mean ergodic convergence in the Calkin algebras for Banach spaces that have the bounded compact approximation property; I obtain (i) a new, coordinate free, characterization of quasidiagonal operators with essential spectra contained in the unit circle by adapting the proof of a classical result in the theory of Banach spaces, (ii) affirmative answers to some questions of Hadwin, and (iii) an alternative proof of Hadwin's characterization of the strong, weak and *-strong operator topologies of the unitary orbit of a given operator on a separable, infinite dimensional, complex Hilbert space; I study appropriately normalized square random Vandermonde matrices based on independent random variables with uniform distribution on the unit circle; and I show that as the matrix size increases without bound, with respect to the expectation of the trace there is an asymptotic *-distribution, equal to that of a $C[0,1]$-valued R-diagonal element.
\end{abstract}




\section{ACKNOWLEDGEMENTS}

I am deeply grateful to my advisors W. B. Johnson and D. Kerr as well as C. Foias and K. Dykema for all the valuable support they gave me. I also thank R. Douglas, C. Pearcy and D. Voiculescu for many very helpful mathematical conversations and T. Cahill, G. Pisier and Th. Schlumprecht for their time and effort spent on me. Section 4 of this dissertation arises from the question about $R$-diagonality of random Vandermonde matrices proposed by G. Tucci. The conversations with my parents and my brother H. Boedihardjo are very important. My research is supported in part by NSF grant DMS-1301604 and the N. W. Naugle Fellowship. 


\section{TABLE OF CONTENTS}

Page

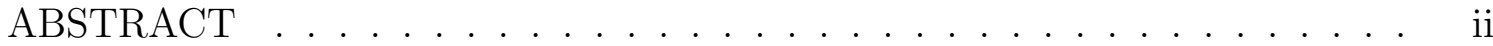

ACKNOWLEDGEMENTS ..................... . . iii

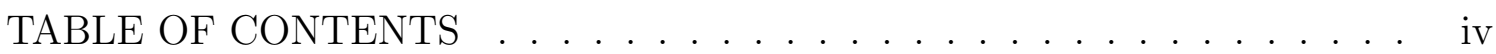

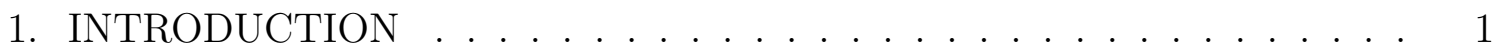

2. MEAN ERGODIC CONVERGENCE . . . . . . . . . . . . . . 4

2.1 Introduction . . . . . . . . . . . . . . . . . . 4

2.2 The Calkin representation for Banach spaces . . . . . . . . . . . . 6

3. A PARALLEL BETWEEN BANACH SPACES AND OPERATORS . . . 15

3.1 Introduction . . . . . . . . . . . . . . . . . . . 15

3.2 Universal Banach spaces and universal operators . . . . . . . . . . . 21

3.3 Ultraproducts of operators . . . . . . . . . . . . . . . . . . . . 29

4. ASYMPTOTIC *-MOMENTS OF SOME RANDOM VANDERMONDE

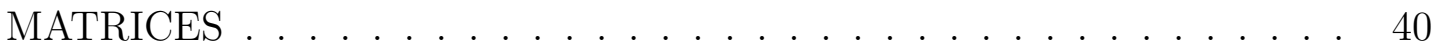

4.1 Introduction . . . . . . . . . . . . . . . . . . 40

4.2 Asymptotic alternating $C[0,1]$-valued $*$-moments. . . . . . . . . . . . 43

$4.3 C[0,1]$-valued R-diagonality . . . . . . . . . . . . . . 51

4.4 Calculating $\Lambda_{\pi}$ and certain moments and cumulants . . . . . . . . . 83

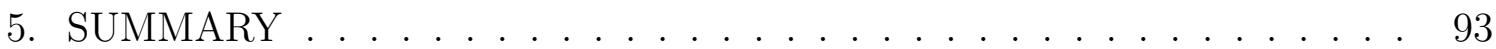

5.1 Mean ergodic convergence . . . . . . . . . . . . . . . . . . 93

5.2 Universal operators . . . . . . . . . . . . . . . . . . . . . . . . . . . . . . . . . . . . 93

5.3 Approximate similarity . . . . . . . . . . . . . . . . . . . 93

5.4 Unitary orbits . . . . . . . . . . . . . . . . . . . . . 93

5.5 Random Vandermonde matrices . . . . . . . . . . . . . . . . . 94

REFERENCES . . . . . . . . . . . . . . . . . . . . . 95 


\section{INTRODUCTION}

This thesis has three parts that are rather unrelated except that ultraproducts appear in the first two parts. The first part is focused on a problem about operators on Banach spaces. The second part concerns uses of a Replacement Rule from the theory of Banach spaces to operator theory on Hilbert space. The last part is about R-diagonality of certain random matrices.

The mean ergodic theorem of von Neumann [39, Section 144] asserts that the averages of the powers of a contraction $T$ on a Hilbert space converges strongly to the projection onto the space of eigenvectors of $T$ with eigenvalue 1 . This result was extended to reflexive Banach spaces by Lorch [27]. Norm convergence was later studied by Dunford [13]. He gave several characterizations including the condition that $(I-T)^{2}$ has closed range. Later Lin [24] showed that $I-T$ having closed range is also an equivalent condition. This result was improved by Mbekhta and Zemánek [28], who proved that for every fixed $m \geq 1,(I-T)^{m}$ having closed range is also an equivalent condition.

In Section 2 (see [4]), I consider the convergence in the Calkin algebra of the averages of the powers of a contraction on Banach space. The main difficulty is that the eigenvectors of $T$ change when we make a compact perturbation of $T$. To obtain the desired characterization, the following concept is introduced. Let $X$ be a Banach space and let $(P)$ be a property that a subspace $M$ of $X$ may or may not have. We say that a subspace $M \subset X$ is an essentially maximal subspace of $X$ satisfying $(P)$ if it has $(P)$ and if every subspace $M_{0} \supset M$ having property $(P)$ satisfies $\operatorname{dim} M_{0} / M<$ $\infty$. Using this concept, we establish a geometric characterization of mean ergodic convergence in the Calkin algebras for Banach spaces that have the bounded compact 
approximation property (BCAP). The BCAP, defined in Subsection 2.1, is a property that all classical separable Banach spaces (including Hilbert space) have. The idea of the proof is to embed the Calkin algebra setting into the operator setting so that the existence of an essentially maximal subspace on which an operator is compact becomes the condition that the operator has closed range. To do this, one needs to assume the bounded compact approximation property.

Usually the connection between the theory of Banach spaces and the theory of operators on Hilbert space involve the study of spaces of operators as Banach spaces and vice versa, Banach spaces as subspaces of $\mathcal{B}(\mathcal{H})$, or operators on Banach space as generalization of operators on Hilbert space. The purpose of Section 3 (see [5]) is to illustate that new insights into operator theory can also be obtained from the theory of Banach spaces via the following Replacement Rule:

Every Banach space is replaced by an operator, a complemented subspace of a Banach space is replaced by a reducing part of the corresponding operator, and an operator between Banach spaces is replaced by an operator intertwining the corresponding operators.

Using this Replacement Rule, I investigate the analogs in operator theory of (i) the problem of complementable universal Banach spaces and (ii) ultraproducts of Banach spaces.

The complementable universality problem for Banach spaces and an analogous problem in operator theory are considered. By adapting (via the above Replacement Rule) the proof of a universality result of Johnson and Szankowski in [23], I obtain a coordinate free characterization of quasidiagonal operators with essential spectra contained in the unit circle.

In addition, I answer affirmatively some questions of Hadwin and give an alternative proof of Hadwin's characterization [19] of the strong, weak and $*$-strong closures 
of the unitary orbit of a given operator on Hilbert space.

Sections 2 and 3 concern mainly infinite dimensional aspects of Functional Analysis. In contrast, Section 4 is about the asymptotic behaviour of some random matrices. More specifically, the random Vandermonde matrix $X_{N}$, whose $(i, j)$-th entry is $N^{-1 / 2} \zeta_{i}^{j}$, where $\zeta_{1}, \ldots, \zeta_{N}$ are independent with uniform distribution on the unit circle, is considered. These random Vandermonde matrices have been studied in [30], [31], [34] and [35] and are interesting in part because of their applications in finance, signal array processing, wireless communications and biology (see [30] for references).

In Section 4 (see [6]), the asymptotic $*$-moments of $X_{N}$, i.e.,

$$
\lim _{N \rightarrow \infty} \mathbb{E} \circ \operatorname{tr} P\left(X_{N}, X_{N}^{*}\right)
$$

are considered, where $\mathbb{E}$ is the expectation and tr is the normalized trace on matrix algebras. These limits are shown to exist. G. Tucci [33] asked whether $X_{N}$ is asymptotically $R$-diagonal with respect to the expectation of the trace. The concept $R$-diagonality belongs to free probability theory. It can be thought of as an ana$\log$ in free probability of rotationally invariant distributions in classical probability. Tucci's question is answered negatively, but the asymptotic *-moments of $X_{N}$ are described using the notion of $R$-diagonality with amalgamation. The main result of this section is that $X_{N}$ has an asymptotic $*$-distribution as $N \rightarrow \infty$, which is in fact the $*$-distribution of an element that is $R$-diagonal over the $C^{*}$-algebra $C[0,1]$. Here we identify functions in $C[0,1]$ with deterministic diagonal matrices in the following way. If $b \in C[0,1]$ then the corresponding deterministic diagonal matrices are

$$
\operatorname{diag}\left(b\left(\frac{1}{N}\right), b\left(\frac{2}{N}\right), \ldots, b\left(\frac{N}{N}\right)\right)
$$




\section{MEAN ERGODIC CONVERGENCE}

This section comes from [4].

\subsection{Introduction}

Let $X$ be a real or complex Banach space and let $B(X)$ be the algebra of all bounded linear operators on $X$. Suppose that $T \in B(X)$ and consider the sequence

$$
M_{n}(T):=\frac{I+T+\ldots+T^{n}}{n+1}, \quad n \geq 1 .
$$

In [13], Dunford considered the norm convergence of $\left(M_{n}(T)\right)_{n}$ and established the following characterizations.

Theorem 2.1. Suppose that $X$ is a complex Banach space and that $T \in B(X)$ satisfies $\frac{\left\|T^{n}\right\|}{n} \rightarrow 0$. Then the following conditions are equivalent.

1. $\left(M_{n}(T)\right)_{n}$ converges in norm to an element in $B(X)$.

2. 1 is a simple pole of the resolvent of $T$ or 1 is in the resolvent set of $T$.

3. $(I-T)^{2}$ has closed range.

It was then discovered by Lin in [24] that $I-T$ having closed range is also an equivalent condition. Moreover, Lin's argument worked also for real Banach spaces. This result was later improved by Mbekhta and Zemánek in [28] in which they showed that $(I-T)^{m}$ having closed range, where $m \geq 1$, are also equivalent conditions. More precisely,

Theorem 2.2. Let $m \geq 1$. Suppose that $X$ is a real or complex Banach space and

that $T \in B(X)$ satisfies $\frac{\left\|T^{n}\right\|}{n} \rightarrow 0$. Then the sequence $\left(M_{n}(T)\right)_{n}$ converges in norm to an element in $B(X)$ if and only if $(I-T)^{m}$ has closed range. 
Let $K(X)$ be the closed ideal of compact operators in $B(X)$. If $T \in B(X)$ then its image in the Calkin algebra $B(X) / K(X)$ is denoted by $\dot{T}$. By Dunford's Theorem 2.1 or by an analogous version for Banach algebras (without condition (3)), when $X$ is a complex Banach space and $\frac{\left\|\dot{T}^{n}\right\|}{n} \rightarrow 0$, the convergence of $\left(M_{n}(\dot{T})\right)_{n}$ in the Calkin algebra is equivalent to 1 being a simple pole of the resolvent of $\dot{T}$ or being in the resolvent set of $\dot{T}$. But even if we are given that the limit $\dot{P} \in B(X) / K(X)$ of $\left(M_{n}(\dot{T})\right)_{n}$ exists, there is no obvious geometric interpretation of $\dot{P}$. In the context of Theorems 2.1 and 2.2, if the limit of $\left(M_{n}(T)\right)_{n}$ exists, then it is a projection onto $\operatorname{ker}(I-T)$. In the context of the Calkin algebra, the limit $\dot{P}$ is still an idempotent in $B(X) / K(X)$; hence by making a compact perturbation, we can assume that $P$ is an idempotent in $B(X)$ (see Lemma 2.9 below).

A natural question to ask is: what is the range of $P$ ? Although the range of $P$ is not unique (since $P$ is only unique up to a compact perturbation), it can be thought of as an analog of $\operatorname{ker}(I-T)$ in the Calkin algebra setting. If $T_{0} \in B(X)$ then $\operatorname{ker} T_{0}$ is the maximal subspace of $X$ on which $T_{0}=0$. This suggests that the analog of ker $T_{0}$ in the Calkin algebra setting is the maximal subspace of $X$ on which $T_{0}$ is compact. But the maximal subspace does not exist unless it is the whole space $X$. Thus, we introduce the following concept.

Let $X$ be a Banach space and let $(P)$ be a property that a subspace $M$ of $X$ may or may not have. We say that a subspace $M \subset X$ is an essentially maximal subspace of $X$ satisfying $(P)$ if it has $(P)$ and if every subspace $M_{0} \supset M$ having property $(P)$ satisfies $\operatorname{dim} M_{0} / M<\infty$.

Then the analog of ker $T_{0}$ in the Calkin algebra setting is an essentially maximal subspace of $X$ on which $T_{0}$ is compact. It turns that if such an analog for $I-T$ exists, then it is already sufficient for the convergence of $\left(M_{n}(\dot{T})\right)_{n}$ in the Calkin algebra (at least for a large class of Banach spaces), which is the main result of this 
section.

Before stating this theorem, we recall that a Banach space $Z$ has the bounded compact approximation property (BCAP) if there is a uniformly bounded net $\left(S_{\alpha}\right)_{\alpha \in \Lambda}$ in $K(Z)$ converging strongly to the identity operator $I \in B(Z)$. It is always possible to choose $\Lambda$ to be the set of all finite dimensional subspaces of $Z$ directed by inclusion. If the net $\left(S_{\alpha}\right)_{\alpha \in \Lambda}$ can be chosen so that $\sup _{\alpha \in \Lambda}\left\|S_{\alpha}\right\| \leq \lambda$, then we say that $Z$ has the $\lambda$-BCAP. It is known that if a reflexive space has the BCAP, then the space has the 1-BCAP [16, Theorem 1.5]. For $T \in B(X)$, the essential norm $\|T\|_{e}$ is the norm of $\dot{T}$ in $B(X) / K(X)$.

Theorem 2.3. Let $m \geq 1$. Suppose that $X$ is a real or complex Banach space having the bounded compact approximation property. If $T \in B(X)$ satisfies $\frac{\left\|T^{n}\right\|_{e}}{n} \rightarrow 0$, then the following conditions are equivalent.

1. The sequence $\left(M_{n}(\dot{T})\right)_{n}$ converges in norm to an element in $B(X) / K(X)$.

2. There is an essentially maximal subspace of $X$ on which $(I-T)^{m}$ is compact.

The idea of the proof is to reduce Theorem 2.3 to Theorem 2.2 by constructing a Banach space $\widehat{X}$ and an embedding $f: B(X) / K(X) \rightarrow B(\widehat{X})$ so that if $T \in B(X)$ and if there is an essentially maximal subspace $M$ of $X$ on which $T$ is compact, then $f(\dot{T})$ has closed range, and then applying Theorem 2.2 to $f(\dot{T})$. The BCAP of $X$ is used to show that $f$ is an embedding but is not used in the construction of $\widehat{X}$ and $f$. The construction of $f$ is based on the Calkin representation [8, Theorem 5.5].

\subsection{The Calkin representation for Banach spaces}

In this subsection, $X$ is a fixed infinite dimensional Banach space. Let $\Lambda_{0}$ be the set of all finite dimensional subspaces of $X$ directed by inclusion $\subset$. Then $\left\{\left\{\alpha \in \Lambda_{0}\right.\right.$ : $\left.\left.\alpha \supset \alpha_{0}\right\}: \alpha_{0} \in \Lambda_{0}\right\}$ is a filter base on $\Lambda_{0}$, so it is contained in an ultrafilter $U$ on $\Lambda_{0}$. 
Let $Y$ be an arbitary infinite dimensional Banach space and let $\left(Y^{*}\right)^{U}$ be the ultrapower (see e.g., [11, Chapter 8]) of $Y^{*}$ with respect to $U$. (The ultrafilter $U$ and the directed set $\Lambda_{0}$ do not depend on $Y$.) If $\left(y_{\alpha}^{*}\right)_{\alpha \in \Lambda_{0}}$ is a bounded net in $Y^{*}$, then its image in $\left(Y^{*}\right)^{U}$ is denoted by $\left(y_{\alpha}^{*}\right)_{\alpha, U}$. Consider the (complemented) subspace

$$
\widehat{Y}:=\left\{\left(y_{\alpha}^{*}\right)_{\alpha, U} \in\left(Y^{*}\right)^{U}: w^{*}-\lim _{\alpha, U} y_{\alpha}^{*}=0\right\}
$$

of $\left(Y^{*}\right)^{U}$. Here $w^{*}-\lim _{\alpha, U} y_{\alpha}^{*}$ is the $w^{*}$-limit of $\left(y_{\alpha}^{*}\right)_{\alpha \in \Lambda_{0}}$ through $U$, which exists by the Banach-Alaoglu Theorem.

Whenever $T \in B(X, Y)$, we can define an operator $\widehat{T} \in B(\widehat{Y}, \widehat{X})$ by sending $\left(y_{\alpha}^{*}\right)_{\alpha, U}$ to $\left(T^{*} y_{\alpha}^{*}\right)_{\alpha, U}$. Note that if $K \in K(X, Y)$ then $\widehat{K}=0$, where $K(X, Y)$ denotes the space of all compact operators in $B(X, Y)$.

Theorem 2.4. Suppose that $X$ has the $\lambda$-BCAP. Then the operator $f: B(X) / K(X) \rightarrow B(\widehat{X}), \dot{T} \mapsto \widehat{T}$, is a conjugate linear norm one $(\lambda+1)$-embedding into $B(\widehat{X})$ satisfying

$$
f(\dot{I})=I \text { and } f\left(\dot{T}_{1} \dot{T}_{2}\right)=f\left(\dot{T}_{2}\right) f\left(\dot{T}_{1}\right), \quad T_{1}, T_{2} \in B(X) .
$$

Proof. It is easy to verify that $f$ is a conjugate linear map, $f(\dot{I})=I$, and $f\left(\dot{T}_{1} \dot{T}_{2}\right)=$ $f\left(\dot{T}_{2}\right) f\left(\dot{T}_{1}\right)$ for $T_{1}, T_{2} \in B(X)$. If $T \in B(X)$, then clearly $\|f(\dot{T})\| \leq\|T\|$, and thus we also have $\|f(\dot{T})\| \leq\|T\|_{e}$. Hence $\|f\| \leq 1$. It remains to show that $f$ is a $(\lambda+1)$-embedding (i.e., $\left.\inf _{\|T\|_{e}>1}\|f(\dot{T})\| \geq(\lambda+1)^{-1}\right)$.

To do this, let $T \in B(X)$ satisfy $\|T\|_{e}>1$. Since $X$ has the $\lambda$-BCAP, we can find a net of operators $\left(S_{\alpha}\right)_{\alpha \in \Lambda_{0}} \subset K(X)$ converging strongly to $I$ such that $\sup _{\alpha \in \Lambda_{0}}\left\|S_{\alpha}\right\| \leq \lambda$. Then $\left\|T^{*}\left(I-S_{\alpha}\right)^{*}\right\|=\left\|\left(I-S_{\alpha}\right) T\right\| \geq\|T\|_{e}>1, \alpha \in \Lambda_{0}$. Thus, there exists $\left(x_{\alpha}^{*}\right)_{\alpha \in \Lambda_{0}} \subset X^{*}$ such that $\left\|x_{\alpha}^{*}\right\|=1$ and $\left\|T^{*}\left(I-S_{\alpha}\right)^{*} x_{\alpha}^{*}\right\|>1$ for $\alpha \in \Lambda_{0}$. 
Note that for every $x \in X$,

$$
\limsup _{\alpha \in \Lambda_{0}}\left|\left\langle\left(I-S_{\alpha}\right)^{*} x_{\alpha}^{*}, x\right\rangle\right|=\limsup _{\alpha \in \Lambda_{0}}\left|\left\langle x_{\alpha}^{*},\left(I-S_{\alpha}\right) x\right\rangle\right| \leq \limsup _{\alpha \in \Lambda_{0}}\left\|\left(I-S_{\alpha}\right) x\right\|=0,
$$

and so the net $\left(\left(I-S_{\alpha}\right)^{*} x_{\alpha}^{*}\right)_{\alpha \in \Lambda_{0}}$ converges in the $w^{*}$-topology to 0 . By the construction of $U$, this implies that

$$
w^{*}-\lim _{\alpha, U}\left(I-S_{\alpha}\right)^{*} x_{\alpha}^{*}=0 .
$$

Therefore, due to the definition $f(\dot{T})=\widehat{T}$, we obtain

$$
\begin{aligned}
(1+\lambda)\|f(\dot{T})\| \geq\|f(\dot{T})\| \lim _{\alpha, U}\left\|\left(I-S_{\alpha}\right)^{*} x_{\alpha}^{*}\right\| & =\|f(\dot{T})\|\left\|\left(\left(I-S_{\alpha}\right)^{*} x_{\alpha}^{*}\right)_{\alpha, U}\right\| \\
& \geq\left\|f(\dot{T})\left(\left(I-S_{\alpha}\right)^{*} x_{\alpha}^{*}\right)_{\alpha, U}\right\| \\
& =\lim _{\alpha, U}\left\|T^{*}\left(I-S_{\alpha}\right)^{*} x_{\alpha}^{*}\right\| \geq 1 .
\end{aligned}
$$

It follows that $\|f(\dot{T})\| \geq(1+\lambda)^{-1}$ whenever $\|T\|_{e}>1$.

Remark 1. We do not know whether Theorem 2.4 is true without the hypothesis that $X$ has the BCAP.

Remark 2. The embedding in Theorem 2.4 is an isometry if the approximating net can be chosen so that $\left\|I-S_{\alpha}\right\|=1$ for every $\alpha$. This is the case if, for example, the space $X$ has a 1-unconditional basis. However, we do not know whether the embedding is an isometry if $X=L_{p}(0,1)$ with $p \neq 2$.

If $N$ is a subset of $Y^{*}$, then we can define a subset $N^{\prime}$ of $\widehat{Y}$ by

$$
N^{\prime}:=\left\{\left(y_{\alpha}^{*}\right)_{\alpha, U} \in \widehat{Y}: \lim _{\alpha, U} d\left(y_{\alpha}^{*}, N\right)=0\right\},
$$


where

$$
d\left(y_{\alpha}^{*}, N\right):=\inf _{z^{*} \in N}\left\|y_{\alpha}^{*}-z^{*}\right\|
$$

Lemma 2.5. If $N$ is a $w^{*}$-closed subspace of $Y^{*}$, then for every $\left(y_{\alpha}^{*}\right)_{\alpha, U} \in \widehat{Y}$,

$$
d\left(\left(y_{\alpha}^{*}\right)_{\alpha, U}, N^{\prime}\right) \leq 2 \lim _{\alpha, U} d\left(y_{\alpha}^{*}, N\right)
$$

Proof. Let $a=\lim _{\alpha, U} d\left(y_{\alpha}^{*}, N\right)$. Let $\delta>0$. Then

$$
A:=\left\{\alpha \in \Lambda: d\left(y_{\alpha}^{*}, N\right)<a+\delta\right\} \in U
$$

Whenever $\alpha \in A,\left\|y_{\alpha}^{*}-z_{\alpha}^{*}\right\|<a+\delta$ for some $z_{\alpha}^{*} \in N$. If we take $z_{\alpha}^{*}=0$ for $\alpha \notin A$, then, since $\sup _{\alpha \in \Lambda}\left\|y_{\alpha}^{*}\right\|<\infty$,

$$
\sup _{\alpha \in \Lambda}\left\|z_{\alpha}^{*}\right\|=\sup _{\alpha \in A}\left\|z_{\alpha}^{*}\right\| \leq(a+\delta)+\sup _{\alpha \in A}\left\|y_{\alpha}^{*}\right\|<\infty
$$

As a consequence, $\left(z_{\alpha}^{*}-w^{*}-\lim _{\beta, U} z_{\beta}^{*}\right)_{\alpha, U} \in N^{\prime}$, since $N$ is $w^{*}$-closed. Therefore,

$$
\begin{aligned}
d\left(\left(y_{\alpha}^{*}\right)_{\alpha, U}, N^{\prime}\right) & \leq d\left(\left(y_{\alpha}^{*}\right)_{\alpha, U},\left(z_{\alpha}^{*}-w^{*}-\lim _{\beta, U} z_{\beta}^{*}\right)_{\alpha, U}\right) \\
& =\lim _{\alpha, U}\left\|y_{\alpha}^{*}-z_{\alpha}^{*}+w^{*}-\lim _{\beta, U} z_{\beta}^{*}\right\| \\
& \leq \lim _{\alpha, U}\left\|y_{\alpha}^{*}-z_{\alpha}^{*}\right\|+\left\|w^{*}-\lim _{\beta, U} z_{\beta}^{*}\right\| \\
& \leq(a+\delta)+\left\|w^{*}-\lim _{\beta, U}\left(z_{\beta}^{*}-y_{\beta}^{*}\right)\right\| \\
& \leq(a+\delta)+\lim _{\beta, U}\left\|z_{\beta}^{*}-y_{\beta}^{*}\right\| \leq 2(a+\delta) .
\end{aligned}
$$

But $\delta$ can be arbitarily close to 0 so $d\left(\left(y_{\alpha}^{*}\right)_{\alpha, U}, N^{\prime}\right) \leq 2 a=2 \lim _{\alpha, U} d\left(y_{\alpha}^{*}, N\right)$. 
Proposition 2.6. If $X$ and $Y$ are infinite dimensional Banach spaces and if $T \in$ $B(X, Y)$ has closed range then $\widehat{T} \in B(\widehat{Y}, \widehat{X})$ also has closed range.

Proof. The operator $T$ has closed range so $T^{*}$ also has closed range. Let $c=$ $\inf \left\{\left\|T^{*} y^{*}\right\|: y^{*} \in Y^{*}, d\left(y^{*}, \operatorname{ker} T^{*}\right)=1\right\}>0$. Then by Lemma 2.5, for every $\left(y_{\alpha}^{*}\right)_{\alpha, U} \in \widehat{Y}$,

$$
\left\|\widehat{T}\left(y_{\alpha}^{*}\right)_{\alpha, U}\right\|=\lim _{\alpha, U}\left\|T^{*} y_{\alpha}^{*}\right\| \geq c \lim _{\alpha, U} d\left(y_{\alpha}^{*}, \operatorname{ker} T^{*}\right) \geq \frac{c}{2} d\left(\left(y_{\alpha}^{*}\right)_{\alpha, U},\left(\operatorname{ker} T^{*}\right)^{\prime}\right) .
$$

But obviously $\left(\operatorname{ker} T^{*}\right)^{\prime} \subset \operatorname{ker} \widehat{T}$, and so

$$
\left\|\widehat{T}\left(y_{\alpha}^{*}\right)_{\alpha, U}\right\| \geq \frac{c}{2} d\left(\left(y_{\alpha}^{*}\right)_{\alpha, U}, \operatorname{ker} \widehat{T}\right), \quad\left(y_{\alpha}^{*}\right)_{\alpha, U} \in \widehat{Y}
$$

Hence $\widehat{T}$ has closed range.

Lemma 2.7. Suppose that $X \subset Y$ and that $T \in B(X)$. Let $T_{0} \in B(X, Y), x \mapsto T x$. Then $\widehat{T}_{0} \widehat{Y}=\widehat{T} \widehat{X}$.

Proof. If $\left(y_{\alpha}^{*}\right)_{\alpha, U} \in \widehat{Y}$, then for each $\alpha \in \Lambda$, we have $T_{0}^{*} y_{\alpha}^{*}=T^{*}\left(y_{\alpha \mid X}^{*}\right)$, and $\left(y_{\alpha \mid X}^{*}\right)_{\alpha, U} \in$ $\widehat{X}$. Thus $\widehat{T}_{0}\left(y_{\alpha}^{*}\right)_{\alpha, U}=\left(T_{0}^{*} y_{\alpha}^{*}\right)_{\alpha, U}=\left(T^{*}\left(y_{\alpha \mid X}^{*}\right)\right)_{\alpha, U}=\widehat{T}\left(y_{\alpha \mid X}^{*}\right)_{\alpha, U} \in \widehat{T} \widehat{X}$. Hence $\widehat{T}_{0} \widehat{Y} \subset \widehat{T} \widehat{X}$.

Conversely, if $\left(x_{\alpha}^{*}\right)_{\alpha, U} \in \widehat{X}$ then we can extend each $x_{\alpha}^{*}$ to an element $y_{\alpha}^{*} \in Y^{*}$ such that $\left\|y_{\alpha}^{*}\right\|=\left\|x_{\alpha}^{*}\right\|$. Thus we have $\left(y_{\alpha}^{*}-w^{*}-\lim _{\beta, U} y_{\beta}^{*}\right)_{\alpha, U} \in \widehat{Y}$. Note that

$$
T_{0}^{*}\left(w^{*}-\lim _{\beta, U} y_{\beta}^{*}\right)=w^{*}-\lim _{\beta, U} T_{0}^{*} y_{\beta}^{*}=w^{*}-\lim _{\beta, U} T^{*} x_{\beta}^{*}=T^{*}\left(w^{*}-\lim _{\beta, U} x_{\beta}^{*}\right)=0 .
$$


This implies that

$$
\begin{aligned}
\widehat{T}\left(x_{\alpha}^{*}\right)_{\alpha, U}=\left(T^{*} x_{\alpha}^{*}\right)_{\alpha, U} & =\left(T_{0}^{*} y_{\alpha}^{*}\right)_{\alpha, U} \\
& =\left(T_{0}^{*}\left(y_{\alpha}^{*}-w^{*}-\lim _{\beta, U} y_{\beta}^{*}\right)\right)_{\alpha, U} \\
& =\widehat{T}_{0}\left(y_{\alpha}^{*}-w^{*}-\lim _{\beta, U} y_{\beta}^{*}\right)_{\alpha, U} \in \widehat{T}_{0} \widehat{Y}
\end{aligned}
$$

Therefore $\widehat{T} \widehat{X} \subset \widehat{T}_{0} \widehat{Y}$.

Proposition 2.8. Suppose that $T \in B(X)$ and that there exists an essentially maximal subspace $M$ of $X$ on which $T$ is compact. Then $\widehat{T}$ has closed range.

Proof. Without loss of generality, we may assume that $X$ is a subspace of $Y=\ell_{\infty}(J)$ for some set $J$. Define $T_{0} \in B\left(X, \ell_{\infty}(J)\right), x \mapsto T x$. Then by assumption, there is an essentially maximal subspace $M$ of $X$ on which $T_{0}$ is compact. By [25, Theorem 3.3], there exists $K \in K\left(X, \ell_{\infty}(J)\right)$ such that $K_{\mid M}=T_{0 \mid M}$.

We now show that $T_{0}-K \in B\left(X, \ell_{\infty}(J)\right)$ has closed range. Since $M \subset \operatorname{ker}\left(T_{0}-\right.$ $K)$ and $M$ is an essentially maximal subspace of $X$ on which $T_{0}-K$ is compact, $\operatorname{ker}\left(T_{0}-K\right)$ is an essentially maximal subspace of $X$ on which $T_{0}-K$ is compact.

Let $\pi$ be the quotient map from $X$ onto $X / \operatorname{ker}\left(T_{0}-K\right)$. Define the (one-toone) operator $R: X / \operatorname{ker}\left(T_{0}-K\right) \rightarrow \ell_{\infty}(J), \pi x \mapsto\left(T_{0}-K\right) x$. If $R$ does not have closed range, then by [26, Proposition 2.c.4], $R$ is compact on an infinite dimensional subspace $V$ of $X / \operatorname{ker}\left(T_{0}-K\right)$. Hence, $T_{0}-K$ is compact on $\pi^{-1} V$ and so by the essential maximality of $\operatorname{ker}\left(T_{0}-K\right)$, we have $\operatorname{dim} \pi^{-1} V / \operatorname{ker}\left(T_{0}-K\right)<\infty$. Thus, $V=\pi^{-1} V / \operatorname{ker}\left(T_{0}-K\right)$ is finite dimensional, which contradicts the definition of $V$.

Therefore, $R$ has closed range and so $T_{0}-K$ also has closed range. By Proposition 2.6, $\widehat{T_{0}-K}$ has closed range. But $\widehat{K}=0$ so $\widehat{T}_{0}$ has closed range and by Lemma 2.7 , $\widehat{T}$ has closed range. 
Lemma 2.9. Suppose that $P \in B(X)$ and that $\dot{P}$ is an idempotent in $B(X) / K(X)$. Then $P$ is the sum of an idempotent in $B(X)$ and a compact operator on $X$.

Proof. We first treat the case where the scalar field is $\mathbb{C}$. From Fredholm theory (see e.g. [17, Chapters XI and XVII]), we know that since $\sigma(\dot{P}) \subset\{0,1\}$, the only possible cluster points of $\sigma(P)$ are 0 and 1 . Thus, there exists $0<r<1$ such that $\{z \in \mathbb{C}:|z-1|=r\} \cap \sigma(P)=\emptyset$. Then $\dot{P}=\frac{1}{2 \pi i} \oint_{|z-1|=r}(z \dot{I}-\dot{P})^{-1} d z$ and so $P-\frac{1}{2 \pi i} \oint_{|z-1|=r}(z I-P)^{-1} d z \in K(X)$. But $\frac{1}{2 \pi i} \oint_{|z-1|=r}(z I-P)^{-1} d z$ is an idempotent in $B(X)$ (see e.g. [37, Theorem 2.7]). This completes the proof in the complex case.

If $X$ is a real Banach space, then let $X_{C}$ and $P_{C}$ be the complexifications (see [14, page 266]) of $X$ and $P$, respectively. Thus, $\dot{P}_{C}$ is an idempotent in $B\left(X_{C}\right) / K\left(X_{C}\right)$. Since the only possible cluster points of $\sigma\left(P_{C}\right)$ are 0 and 1 , there exists a closed rectangle $R$ in the complex plane symmetric with respect to the real axis such that 1 is in the interior of $R, 0$ is in the exterior of $R$, and $\sigma\left(P_{C}\right)$ is disjoint from the boundary $\partial R$ of $R$. By [14, Lemma 3.4], the idempotent $\frac{1}{2 \pi i} \oint_{\partial R}\left(z I-P_{C}\right)^{-1} d z$ in $B\left(X_{C}\right)$ is induced by an idempotent $P_{0}$ in $B(X)$. Since $P_{C}-\frac{1}{2 \pi i} \oint_{\partial R}\left(z I-P_{C}\right)^{-1} d z \in K\left(X_{C}\right)$, we see that $P-P_{0} \in K(X)$.

Proof of Theorem 2.3. "(1) $\Rightarrow(2) "$ : Let $\dot{P}:=\lim _{n \rightarrow \infty} \frac{\dot{I}+\dot{T}+\ldots+\dot{T}^{n}}{n+1}$. Since $\lim _{n \rightarrow \infty} \frac{\left\|\dot{T}^{n}\right\|}{n}=0$

$$
(\dot{I}-\dot{T}) \dot{P}=\lim _{n \rightarrow \infty}(\dot{I}-\dot{T}) \frac{\dot{I}+\dot{T}+\ldots+\dot{T}^{n}}{n+1}=\lim _{n \rightarrow \infty} \frac{\dot{I}-\dot{T}^{n+1}}{n+1}=0
$$

Thus $\dot{T} \dot{P}=\dot{P}$, and so

$$
\dot{P}^{2}=\lim _{n \rightarrow \infty} \frac{\dot{P}+\dot{T} \dot{P}+\ldots+\dot{T}^{n} \dot{P}}{n+1}=\lim _{n \rightarrow \infty} \frac{(n+1) \dot{P}}{n+1}=\dot{P} .
$$


Hence $\dot{P}$ is an idempotent in $B(X) / K(X)$. By Lemma 2.9, there exists an idempotent $P_{0}$ in $B(X)$ such that $P-P_{0} \in K(X)$. Replacing $P$ with $P_{0}$, we can assume without loss of generality that $P$ is an idempotent in $B(X)$. Equation (2.1) also implies that $(I-T) P \in K(X)$, which means that $I-T$ is compact on $P X$. Hence $(I-T)^{m}$ is compact on $P X$.

We now show that $P X$ is an essentially maximal subspace of $X$ on which $(I-T)^{m}$ is compact. Suppose that $(I-T)^{m}$ is compact on a subspace $M_{0}$ of $X$ containing $P X$. Let

$$
f_{n}(z):=\frac{n+(n-1) z+(n-2) z^{2}+\ldots+z^{n-1}}{n+1}, \quad z \in \mathbb{C}, n \geq 1
$$

Note that $\dot{I}-\frac{\dot{I}+\dot{T}+\ldots+\dot{T}^{n}}{n+1}=(\dot{I}-\dot{T}) f_{n}(\dot{T})$. Therefore,

$$
\dot{I}-\dot{P}=(\dot{I}-\dot{P})^{m}=\lim _{n \rightarrow \infty} f_{n}(\dot{T})^{m}(\dot{I}-\dot{T})^{m}
$$

and so

$$
\lim _{n \rightarrow \infty}\left\|(I-P)-\left(f_{n}(T)^{m}(I-T)^{m}+K_{n}\right)\right\|=0
$$

for some $K_{1}, K_{2}, \ldots \in K(X)$.

Since $(I-T)^{m}$ is compact on $M_{0}$, the operator $f_{n}(T)^{m}(I-T)^{m}$ is compact on $M_{0}$ and so is $f_{n}(T)^{m}(I-T)^{m}+K_{n}$ on $M_{0}$. Thus $(I-P)_{\mid M_{0}}$ is the norm limit of a sequence in $K\left(M_{0}, X\right)$, and so $I-P$ is compact on $M_{0}$. Since $P X \subset M_{0}$, we have that $(I-P) M_{0} \subset M_{0}$. Therefore, $(I-P)_{\mid(I-P) M_{0}}=I_{\mid(I-P) M_{0}}$ is compact, and so $(I-P) M_{0}$ is finite dimensional. In other words, $\operatorname{dim} M_{0} / P X<\infty$.

"(2) $\Rightarrow(1) "$ : By Proposition 2.8, $\left(\widehat{I-T)^{m}}=(I-\widehat{T})^{m}\right.$ has closed range. Since by assumption $\lim _{n \rightarrow \infty} \frac{\left\|T^{n}\right\|_{e}}{n}=0, \lim _{n \rightarrow \infty} \frac{\left\|\widehat{T}^{n}\right\|}{n}=\lim _{n \rightarrow \infty} \frac{\left\|\widehat{T^{n}}\right\|}{n}=0$. By Mbekhta-Zemánek's Theorem 2.2, the sequence $\left(M_{n}(\widehat{T})\right)_{n}$ converges in norm to an element in $B(\widehat{X})$. By 
Theorem 2.4, the result follows. 


\section{A PARALLEL BETWEEN BANACH SPACES AND OPERATORS}

This section comes from [5].

\subsection{Introduction}

In this subsection, $\mathcal{H}$ is always a fixed separable, infinite dimensional, complex Hilbert space, and $\mathcal{B}(\mathcal{H})$ is the algebra of all operators (i.e., bounded linear transformations) on $\mathcal{H}$. The ideal of compact operators in $\mathcal{B}(\mathcal{H})$ is denoted by $\mathcal{K}(\mathcal{H})$. If $\mathcal{H}_{1}$ and $\mathcal{H}_{2}$ are Hilbert spaces, then $\mathcal{B}\left(\mathcal{H}_{1}, \mathcal{H}_{2}\right)$ denotes the set of all operators from $\mathcal{H}_{1}$ into $\mathcal{H}_{2}$.

Usually the connection between the theory of Banach spaces and the theory of operators on Hilbert space involve the study of spaces of operators as Banach spaces and vice versa, Banach spaces as subspaces of $\mathcal{B}(\mathcal{H})$, or operators on Banach space as generalization of operators on Hilbert space. The purpose of this section is to illustate that new insights into operator theory can also be obtained from the theory of Banach spaces via the following Replacement Rule:

Every Banach space is replaced by an operator, a complemented subspace of a Banach space is replaced by a reducing part of the corresponding operator, and an operator between Banach spaces is replaced by an operator intertwining the corresponding operators.

Using this Replacement Rule, we investigate the analogs in operator theory of (i) the problem of complementable universal Banach spaces and (ii) ultraproducts of Banach spaces. The main consequences of this investigation are

(I) a coordinate free characterization of quasidiagonal operators with essential spectra contained in the unit circle (i.e., a characterization that does not require one to find a decomposition of the space into finite dimensional subspaces 
or to find an appropriate sequence of projections converging strongly to the identity in order to determine that a given operator with essential spectrum contained in the unit circle is quasidiagonal.);

(II) the following result: Suppose that $T_{1}, T_{2} \in \mathcal{B}(\mathcal{H})$. If $\lambda \geq 1$ and

$$
T_{2} \in\left\{S T_{1} S^{-1}: S \in \mathcal{B}(\mathcal{H}) \text { with }\|S\|\left\|S^{-1}\right\| \leq \lambda\right\}^{-\|\|}
$$

then there exists a sequence $\left(S_{n}\right)_{n \geq 1}$ of invertible operators on $\mathcal{H}$ with $\left\|S_{n}\right\|\left\|S_{n}^{-1}\right\| \leq \lambda$ such that $\lim _{n \rightarrow \infty}\left\|T_{2}-S_{n} T_{1} S_{n}^{-1}\right\|=0$ and $T_{2}-S_{n} T_{1} S_{n}^{-1} \in \mathcal{K}(\mathcal{H})$; and

(III) an alternative proof of Hadwin's characterization [19] of the SOT, WOT and *-SOT closure of the unitary orbit of a given operator on $\mathcal{H}$.

The author later became aware that the proof of (II) answers affirmatively the following questions of Hadwin (see Question 1 and 9 in [20].)

Hadwin defined two operators $T_{1}, T_{2} \in \mathcal{B}(\mathcal{H})$ to be approximately similar if there exists $\lambda \geq 1$ satisfying (3.1) above. He asked whether or not $T_{1}, T_{2} \in \mathcal{B}(\mathcal{H})$ are approximately similar (if and) only if there exist $n \geq 1$ and $B_{1}, B_{2}, \ldots, B_{n} \in \mathcal{B}(\mathcal{H})$ such that $B_{1}=T_{1}, B_{n}=T_{2}$ and for each $1 \leq k<n, B_{k}$ is either similar or approximately unitarily equivalent to $B_{k+1}$. Hadwin also asked that in case this is true, can we find one $n \geq 1$ that is valid for all $T_{1}$ and $T_{2}$ that are approximately similar.

He pointed out that if there exists such $n \geq 1$, it has to be at least 4 (see Example 1 in [20]) and that $n=4$ is valid under the assumption that the unital $C^{*}$-algebra generated by $T_{1}$ and the unital $C^{*}$-algebra generated by $T_{2}$ are both disjoint from $\mathcal{K}(\mathcal{H})$ except for 0 . From the proof of (II), we obtain that $n=4$ is valid without this 
assumption. Indeed, we obtain that if $T_{1}$ and $T_{2}$ are approximately similar, then $T_{1}$ is approximately unitarily equivalent to an operator $T_{1}^{\prime} \in \mathcal{B}(\mathcal{H})$ that is similar to an operator $T_{2}^{\prime} \in \mathcal{B}(\mathcal{H})$ that is approximately unitarily equivalent to $T_{2}$.

In Subsection 2, we consider the complementably universality problem for Banach spaces (see Problem 1 below), and we find an analogous problem in operator theory (see Problem 3 below). Then by adapting (via the above Replacement Rule) the proof of a universality result of Johnson and Szankowski in [23], we obtain a partial solution to Problem 3 (see Theorem 3.5). This partial solution yields (I) above (see Corollary 3.9).

In Subsection 3, we consider ultraproducts of operators on Hilbert space, and use them, together with the Calkin representation [8] and Voiculescu's theorem [41], to obtain (II) and (III) above (see Theorem 3.16 and Theorem 3.17, respectively). The connection of the results in this subsection to the theory of Banach spaces is not clear at all at the first glance. But all the results were indeed inspired by ultraproducts of Banach spaces and the closely related concept of finite representability of Banach spaces. See the end of the subsection. I later became aware that the technique used in this subsection is similar to that used in [15, Section 3].

We begin by introducing some terminology and notation that will be needed in what follows.

Subspaces are always assumed to be norm closed. Throughout this chapter, we will systematically use the symbols $X, Y, Z$ for Banach spaces, $A, B, S, T$ for operators, $K$ for a compact operator, $W$ for a unitary operator between Hilbert spaces, $P, Q$ for idempotents, and $I$ for the identity operator on a Banach space. 


\section{A. Operator theory}

Let $T_{1} \in \mathcal{B}\left(\mathcal{H}_{1}\right)$ and $T_{2} \in \mathcal{B}\left(\mathcal{H}_{2}\right)$. An operator $A \in \mathcal{B}\left(\mathcal{H}_{1}, \mathcal{H}_{2}\right)$ intertwines $T_{1}$ and $T_{2}$ if $A T_{1}=T_{2} A$.

The operators $T_{1}$ and $T_{2}$ are compalent [36], denoted by $T_{1} \stackrel{c}{\sim} T_{2}$, if there exist a unitary operator $W \in \mathcal{B}\left(\mathcal{H}_{1}, \mathcal{H}_{2}\right)$ and a compact operator $K \in \mathcal{K}\left(\mathcal{H}_{2}\right)$ such that

$$
T_{2}=W T_{1} W^{*}+K
$$

$T_{1}$ and $T_{2}$ are approximately unitarily equivalent [41], denoted by $T_{1} \simeq_{a} T_{2}$, if there exists a sequence $\left(W_{n}\right)_{n \geq 1}$ of unitary operators in $\mathcal{B}\left(\mathcal{H}_{1}, \mathcal{H}_{2}\right)$ such that $T_{2}-W_{n} T_{1} W_{n}^{*} \in$ $\mathcal{K}\left(\mathcal{H}_{2}\right)$ for all $n \geq 1$ and

$$
\lim _{k \rightarrow \infty}\left\|T_{2}-W_{k} T_{1} W_{k}^{*}\right\|=0
$$

$T_{1}$ and $T_{2}$ are unitarily equivalent, denoted by $T_{1} \cong T_{2}$, if there exists a unitary operator $W \in \mathcal{B}\left(\mathcal{H}_{1}, \mathcal{H}_{2}\right)$ such that $T_{2}=W T_{1} W^{*}$. The unitary orbit of an operator $T \in \mathcal{B}(\mathcal{H})$ is defined by

$$
\mathcal{U}(T):=\left\{T_{0} \in \mathcal{B}(\mathcal{H}): T \cong T_{0}\right\}
$$

Let $T \in \mathcal{B}(\mathcal{H})$ and let $\mathcal{M}$ be a subspace of $\mathcal{H}$. An operator $T_{0} \in \mathcal{B}(\mathcal{M})$ is a restriction of $T$ if $\mathcal{M}$ is invariant under $T$ and $T_{0}=\left.T\right|_{\mathcal{M}} ; T_{0}$ is a reducing part of $T$ if moreover $\mathcal{M}$ is a reducing subspace for $T$, i.e., invariant under $T$ and $T^{*} ; T_{0}$ is a compression of $T$ if $T_{0}=\left.P T\right|_{\mathcal{M}}$, where $P$ is the orthogonal projection from $\mathcal{H}$ onto $\mathcal{M}$

The operator $T$ is block diagonal [21] if it is unitarily equivalent to a countably infinite direct sum of operators each of which acts on a finite dimensional Hilbert 
space; $T$ is quasidiagonal [21] if it is the sum of a block diagonal operator and a compact operator; $T$ is subnormal if it is the restriction of a normal operator; $T$ is contractive if $\|T\| \leq 1$. A contractive operator is called a contraction.

Let $\pi$ be the quotient map from $\mathcal{B}(\mathcal{H})$ onto $\mathcal{B}(\mathcal{H}) / \mathcal{K}(\mathcal{H})$. We write $\|T\|_{e}:=$ $\|\pi(T)\|$ and $\sigma_{e}(T):=\sigma(\pi(T))$ for the essential norm and the essential spectrum of $T$, respectively. A representation $\rho$ on a Hilbert space $\mathcal{H}_{0}$ of a unital $C^{*}$-algebra $\mathcal{A}$ is a $*$-homomorphism from $\mathcal{A}$ into $\mathcal{B}\left(\mathcal{H}_{0}\right)$. We say that $\rho$ is unital if $\rho(1)=I$. If $a \in \mathcal{A}$ then the unital $C^{*}$-subalgebra of $\mathcal{A}$ generated by $a$ is denoted by $C^{*}(a)$.

As usual, the strong operator topology is denoted by SOT and the weak operator topology is denoted by WOT. A net $\left\{T_{\alpha}\right\}_{\alpha \in \Lambda}$ of operators in $\mathcal{B}(\mathcal{H})$ converges in the *-strong operator topology if both $T_{\alpha} \rightarrow T$ and $T_{\alpha}^{*} \rightarrow T^{*}$ in SOT. This topology is denoted by $*$-SOT.

The following known lemmas are stated here for the reader's convenience.

Lemma 3.1 ([21], page 903). Every contractive quasidiagonal opeator is the sum of a contractive block diagonal operator and a compact operator.

Lemma 3.2 ([40], Corollary 3.3). Let $\mathcal{H}_{1}$ and $\mathcal{H}_{2}$ be complex Hilbert spaces. If $T_{1} \in \mathcal{B}\left(\mathcal{H}_{1}\right)$ and $T_{2} \in \mathcal{B}\left(\mathcal{H}_{2}\right)$ have disjoint essential spectra and $A \in \mathcal{B}\left(\mathcal{H}_{1}, \mathcal{H}_{2}\right)$ intertwines $T_{1}$ and $T_{2}$, then $A$ is compact.

The following proposition may not have been noticed before and will be used in Theorem 3.5.

Proposition 3.3. Let $A \in \mathcal{B}(\mathcal{H})$. If $I \in\left\{K \in \mathcal{K}(\mathcal{H}): K A=A K, K^{*}=K\right\}^{-S O T}$ then $A$ is block diagonal.

Proof. By assumption, there is a net $\left(K_{\alpha}\right)$ of self-adjoint compact operators on $\mathcal{H}$ commuting with $A$ converging in SOT to $I$. Let $\left(\epsilon_{\alpha}\right)$ be a net of positive numbers 
converging to 0 (e.g., take $\epsilon_{\alpha}=\sum_{n=1}^{\infty} \frac{1}{2^{n}} \min \left(\left\|\left(K_{\alpha}-I\right) x_{n}\right\|, 1\right)$ where $\left(x_{n}\right)_{n \geq 1}$ is a dense sequence in the unit ball of $\mathcal{H}$ )

Let $E$ be the spectral measure of $K_{\alpha}$ and let $P_{\alpha}=E\left(\mathbb{R} \backslash\left[-\epsilon_{\alpha}, \epsilon_{\alpha}\right]\right)$, the spectral projection of the given set. Note that $P_{\alpha}$ commutes with $A$, since $P_{\alpha}$ is a sum of orthogonal projections onto $\operatorname{ker}\left(K_{\alpha}-\lambda I\right)$ and $K_{\alpha}$ commutes with both $A$ and $A^{*}$. Since $P_{\alpha} x$ is the best approximation of $x$ by elements of $P_{\alpha} \mathcal{H}$,

$$
\begin{aligned}
\left\|x-P_{\alpha} x\right\| \leq\left\|x-P_{\alpha} K_{\alpha} x\right\| & \leq\left\|x-K_{\alpha} x\right\|+\left\|\left(I-P_{\alpha}\right) K_{\alpha} x\right\| \\
& =\left\|x-K_{\alpha} x\right\|+\left\|E\left(\left[-\epsilon_{\alpha}, \epsilon_{\alpha}\right]\right) K_{\alpha} x\right\| \\
& \leq\left\|x-K_{\alpha} x\right\|+\epsilon_{\alpha}\|x\| \rightarrow 0,
\end{aligned}
$$

for all $x \in \mathcal{H}$. Thus, $P_{\alpha} \rightarrow I$ in SOT. But $P_{\alpha}$ commutes with $A$ and $P_{\alpha} \mathcal{H}$ is finite dimensional (by compactness of $K_{\alpha}$ ). Therefore,

$I \in\{P \in \mathcal{B}(\mathcal{H}): P \text { is a finite rank orthogonal projection and } P A=A P\}^{-S O T}$.

Since this set of $P$ is uniformly bounded, there exists a sequence $\left(P_{n}\right)_{n \geq 1}$ of finite rank orthogonal projections converging in SOT to $I \in \mathcal{B}(\mathcal{H})$ and commuting with $A$. Let $Q_{n}$ be the orthogonal projection from $\mathcal{H}$ onto the closed subspace of $\mathcal{H}$ generated by $P_{1} \mathcal{H} \cup \ldots \cup P_{n} \mathcal{H}$. Then $Q_{n} \rightarrow I$ in SOT, and $Q_{k} \leq Q_{k+1}$ and $Q_{k}$ commutes with $A$ for all $k \geq 1$. Hence, $A$ is block diagonal.

Remark. One consequence of this result is that a reducing part of a block diagonal operator is also block diagonal, which is perhaps a known fact. 


\section{B. Theory of Banach spaces}

Two Banach spaces $X$ and $Y$ are isomorphic if there is an isomorphism from $X$ onto $Y$, i.e., a linear homeomorphism from $X$ onto $Y$. A subspace $Z$ of $X$ is said to be complemented if there is an idempotent from $X$ onto $Z$. We say that the Banach space $X$ has the bounded compact approximation property $(B C A P)$ if there is a uniformly bounded net of compact operators on $X$ converging in SOT to $I$.

General results in operator theory can be found in [12] and [37]. For an introduction to compalence of operators, the reader is referred to [36]. General results in the theory of Banach spaces can be found in [26] and [11].

\subsection{Universal Banach spaces and universal operators}

The motivation for the work in this subsection derives from the following classical problem in the theory of Banach spaces.

Problem 1. For a given class $\mathcal{C}$ of separable Banach spaces, does there exist a separable Banach space $X$ such that every Banach space in $\mathcal{C}$ is isomorphic to a complemented subspace of $X$ ?

The Replacement Rule introduced at the beginning of this subsection suggests that an analog of this problem in operator theory could be

Problem 2. For a given class $\mathcal{C}$ of uniformly bounded operators in $\mathcal{B}(\mathcal{H})$ (i.e., $\sup \{\|T\|: T \in \mathcal{C}\}<\infty)$, does there exist an operator $T \in \mathcal{B}(\mathcal{H})$ such that every operator in $\mathcal{C}$ is unitarily equivalent to a reducing part of $T$ ?

The answer is trivially yes, if $\mathcal{C}$ is countable, by considering the direct sum of all operators in $\mathcal{C}$. On the other hand, the answer is no even for the class $\{\alpha I: \alpha \in[0,1]\}$ (which is uncountable). To see this, suppose that $T \in \mathcal{B}(\mathcal{H})$ and that for every 
$\alpha \in[0,1]$, there is an infinite dimensional reducing subspace $\mathcal{H}_{\alpha}$ for $T$ such that $\left.T\right|_{\mathcal{H}_{\alpha}}=\alpha I$. Letting $P_{\alpha}$ be the orthogonal projection onto $\mathcal{H}_{\alpha}$, we have

$$
T P_{\alpha} P_{\beta}=\left.T\right|_{\mathcal{H}_{\alpha}} P_{\alpha} P_{\beta}=\alpha P_{\alpha} P_{\beta}
$$

Similarly, we have $P_{\alpha} T P_{\beta}=\beta P_{\alpha} P_{\beta}$. But since $T$ commutes with $P_{\alpha}$, it follows that $P_{\alpha} P_{\beta}=0$ if $\alpha \neq \beta$. Therefore, $\mathcal{H}_{\alpha} \perp \mathcal{H}_{\beta}$ if $\alpha \neq \beta$. Since there are uncountably many $\alpha$, this implies that $\mathcal{H}$ is not separable, which is a contradiction.

In general, the answer to Problem 2 is no. Thus, we might obtain a more interesting problem if we replace unitary equivalence with a weaker equivalence, namely with compalence.

Problem 3. For a given class $\mathcal{C}$ of uniformly bounded operators in $\mathcal{B}(\mathcal{H})$, does there exist an operator $T \in \mathcal{B}(\mathcal{H})$ such that every operator in $\mathcal{C}$ is compalent to a reducing part of $T$ ?

Is there, for instance, an operator $T \in \mathcal{B}(\mathcal{H})$ for which every multiple of $I \in \mathcal{B}(\mathcal{H})$ by a scalar in $[0,1]$ is compalent to a reducing part of $T$ ?

The answer is yes. An example is given by a diagonal operator $T$ with diagonal entries $\alpha_{1}, \alpha_{2}, \ldots$ in $\mathbb{R}$ satisfying $\left\{\alpha_{n}: n \geq 1\right\}^{-}=[0,1]$. Then for each $\alpha \in[0,1]$, there is a subsequence $\left(\alpha_{n_{k}}\right)_{k \geq 1}$ converging to $\alpha$. Hence, $\alpha I$ is a compact perturbation of a diagonal operator $B$ with diagonal entries $\alpha_{n_{1}}, \alpha_{n_{2}}, \ldots$ But $B$ is (unitarily equivalent to) a reducing part of $T$, and therefore, $\alpha I$ is compalent to a reducing part of $T$. Is there an operator $T \in \mathcal{B}(\mathcal{H})$ for which every multiple of the unilateral shift (of multiplicity 1 ) by a scalar in $[0,1]$ is compalent to a reducing part of $T$ ? (See Corollary 3.10 below.) What about the bilateral shift?

For the class $(C Q D)$ of contractive quasidiagonal operators, we give below an affirmative answer to Problem 3. This yields, in particular, an affirmative answer 
to the preceding question about the bilateral shift, since every normal operator is quasidiagonal [21, page 903].

Theorem 3.4. There is a contractive quasidiagonal operator $T \in \mathcal{B}(\mathcal{H})$ such that every contractive quasidiagonal operator is compalent to a reducing part of $T$.

Proof. For each $n \geq 1$, let $\left(T_{i, n}\right)_{i \geq 1}$ be a dense sequence in the unit ball of $\mathcal{B}\left(\mathcal{H}_{n}\right)$, where $\mathcal{H}_{n}$ is an $n$-dimensional Hilbert space. Then set

$$
T=\bigoplus_{i, n \geq 1} T_{i, n}
$$

If $A$ is a contractive quasidiagonal operator then by Lemma $3.1, A$ is a compact perturbation of a contractive block diagonal operator $B$. It can be easily checked that $B$ is compalent to a reducing part of $T$. Therefore, $A$ is compalent to a reducing part of $T$.

Remark. The construction of $T$ in Theorem 3.4 is the same as the construction of the universal operator in [22, Corollary 4.2]. But the notion of universality in [22, Corollary 4.2], when restricted to $(C Q D)$, is weaker than that in Theorem 3.4.

The main result of this subsection is that under an additional assumption, the quasidiagonal operators actually characterize the existence of a universal operator.

Theorem 3.5. Suppose that $\mathcal{C}$ is a collection of uniformly bounded operators in $\mathcal{B}(\mathcal{H})$ such that $\sigma_{e}\left(S_{1}\right) \cap \sigma_{e}\left(S_{2}\right)=\emptyset$ for all $S_{1}, S_{2} \in \mathcal{C}, S_{1} \neq S_{2}$. Then the following statements are equivalent.

(i) There exists an operator $T \in \mathcal{B}(\mathcal{H})$ such that every operator in $\mathcal{C}$ is compalent to a reducing part of $T$.

(ii) Every operator in $\mathcal{C}$ outside a countable subset is quasidiagonal. 
Remarks. In Theorem 3.5, we can replace uniform boundedness of $\mathcal{C}$ by essential uniform boundedness, i.e., $\sup \left\{\|T\|_{e}: T \in \mathcal{C}\right\}<\infty$. A slightly stronger statement of Theorem 3.4 is also true: There is a contractive quasidiagonal operator $T \in \mathcal{B}(\mathcal{H})$ such that every quasidiagonal operator with essential norm at most 1 is compalent to a reducing part of $T$.

The universality result mentioned in the introduction that led to Theorem 3.5 is the following.

Theorem 3.6 ([23], Section II). There is no separable Banach space $X$ such that every separable Banach space is isomorphic to a complemented subspace of X.

The proof in [23] uses the following fact about Banach spaces: There are separable Banach spaces $E_{p}$ where $1<p<\infty$ such that (a) $E_{p}$ fails the BCAP for every $1<p<\infty$ and (b) if $q<r$ then every operator from a subspace of $E_{r}$ into $E_{q}$ is compact. Then the result follows from the following lemma. (This lemma is in fact not stated in [23] but is extracted from the original proof of Theorem 3.6 in [23].)

Lemma 3.7. Suppose that $E_{p}$ is a separable Banach space where $1<p<\infty$ such that

(a) $E_{p}$ fails the BCAP for each $1<p<\infty$, and

(b) if $q<r$ then every operator from $E_{r}$ to $E_{q}$ is compact.

Then there is no separable Banach space $X$ such that for every $1<p<\infty, E_{p}$ is isomorphic to a complemented subspace of $X$.

Proof. Suppose, on the contrary, that there is a separable Banach space $X$ such that for every $1<p<\infty, E_{p}$ is isomorphic to a complemented subspace $Y_{p}$ of $X$. Letting 
$Q_{p}$ be an idempotent from $X$ onto $Y_{p}$, we have that there exist $M \in \mathbb{N}$ and an uncountable set $\mathscr{A} \subset(1, \infty)$ so that $\left\|Q_{p}\right\| \leq M$ for each $p \in \mathscr{A}$.

For each $p \in \mathscr{A}$, since $E_{p}$ fails the BCAP, $Y_{p}$ fails the BCAP so $I \notin\left\{K: Y_{p} \rightarrow\right.$ $Y_{p}: K$ is compact and $\left.\|K\| \leq M^{2}\right\}^{-S O T}$. Thus, there is an SOT-open neighborhood of $I$ on $Y_{p}$ that is disjoint from $\left\{K: Y_{p} \rightarrow Y_{p}: K\right.$ is compact and $\left.\|K\| \leq M^{2}\right\}$. By the definition of SOT, this means that there exist a finite set $\left(y_{i}^{p}\right)_{i=1}^{n(p)}$ of unit vectors in $Y_{p}$ and $\epsilon_{p}>0$ so that there is no compact operator $K$ on $Y_{p}$ for which $\|K\| \leq M^{2}$ and $\left\|y_{i}^{p}-K y_{i}^{p}\right\|<\epsilon_{p}$ for $1 \leq i \leq n(p)$. Choose an uncountable subset $\mathscr{B}$ of $\mathscr{A}$ so that $n(p)$ is constant (say $=n$ ) on $\mathscr{B}$ and $\inf _{p \in \mathscr{B}} \epsilon_{p}=\epsilon>0$.

Since $\mathscr{B}$ is uncountable and $X$ is separable, there exist $q<r$ in $\mathscr{B}$ so that $\left\|y_{i}^{q}-y_{i}^{r}\right\|<\left(M+M^{2}\right)^{-1} \epsilon$ for $1 \leq i \leq n$. Let $K_{0}: Y_{r} \rightarrow Y_{r}$ be the restriction of $Q_{r} Q_{q}$ to $Y_{r}$. Then the following properties of $K_{0}$ are valid.

(i) $K_{0}$ is compact, since $\left.Q_{q}\right|_{Y_{r}}: Y_{r} \rightarrow Y_{q}$ is compact by assumption (b);

(ii) $\left\|K_{0}\right\| \leq M^{2}$; and

(iii) $\left\|y_{i}^{r}-K_{0} y_{i}^{r}\right\|<\epsilon_{r}$ for $1 \leq i \leq r$. Indeed,

$$
\begin{aligned}
\left\|y_{i}^{r}-K_{0} y_{i}^{r}\right\| & =\left\|y_{i}^{r}-Q_{r} Q_{q} y_{i}^{r}\right\| \\
& =\left\|Q_{r} y_{i}^{r}-Q_{r} Q_{q} y_{i}^{r}\right\| \\
& \leq M\left\|\left(I-Q_{q}\right) y_{i}^{r}\right\| \\
& =M\left\|\left(I-Q_{q}\right)\left(y_{i}^{r}-y_{i}^{q}\right)\right\| \leq M(1+M)\left\|y_{i}^{r}-y_{i}^{q}\right\|<\epsilon \leq \epsilon_{r} .
\end{aligned}
$$

These properties of $K_{0}$ contradict the choice of $\left(y_{i}^{r}\right)_{i=1}^{n}$ and the proof is complete.

The preceding lemma can be adapted to the context of operator theory via the Replacement Rule. 
Lemma 3.8. Suppose that $\left(S_{\alpha}\right)_{\alpha \in \Lambda}$ is an uncountable indexed collection of nonquasidiagonal operators such that

(a) $S_{\alpha}$ is not quasidiagonal for each $\alpha \in \Lambda$ and

(b) if $\beta \neq \gamma$ then every operator intertwining $S_{\beta}$ and $S_{\gamma}$ is compact.

Then there is no operator $T \in \mathcal{B}(\mathcal{H})$ such that for every $\alpha \in \Lambda, S_{\alpha}$ is compalent to a reducing part of $T$.

Proof. Suppose, on the contrary, that there is an operator $T \in \mathcal{B}(\mathcal{H})$ such that for every $\alpha \in \Lambda, S_{\alpha}$ is compalent to a reducing part $T_{\alpha}:=\left.T\right|_{\mathcal{H}_{\alpha}}$ of $T$ where $\mathcal{H}_{\alpha}$ is a reducing subspace for $T$. Let $P_{\alpha}$ be the orthogonal projection from $\mathcal{H}$ onto $\mathcal{H}_{\alpha}$.

For each $\alpha \in \Lambda$, since $S_{\alpha}$ is not quasidiagonal, $T_{\alpha}$ is not block diagonal, so by Proposition 3.3, $I \notin\left\{K \in \mathcal{K}(\mathcal{H}): K T_{\alpha}=T_{\alpha} K \text { and } K^{*}=K\right\}^{-S O T}$. Thus, there is an SOT-open neighborhood of $I \in \mathcal{B}(\mathcal{H})$ that is disjoint from $\left\{K \in \mathcal{K}(\mathcal{H}): K T_{\alpha}=\right.$ $T_{\alpha} K$ and $\left.K^{*}=K\right\}$. By definition of SOT, this means that there exist a finite set $\left(x_{i}^{\alpha}\right)_{i=1}^{n(\alpha)}$ of unit vectors in $\mathcal{H}_{\alpha}$ and $\epsilon_{\alpha}>0$ so that there is no self-adjoint compact operator $K$ on $\mathcal{H}_{\alpha}$ commuting with $T_{\alpha}$ for which $\left\|x_{i}^{\alpha}-K x_{i}^{\alpha}\right\|<\epsilon_{\alpha}$ for $1 \leq i \leq n(\alpha)$. Choose an uncountable subset $\mathscr{B}$ of $\Lambda$ so that $n(\alpha)$ is constant (say $=n$ ) on $\mathscr{B}$ and $\inf _{\alpha \in \mathscr{B}} \epsilon_{\alpha}=\epsilon>0$.

Since $\mathscr{B}$ is uncountable and $\mathcal{H}$ is separable, there exist $\beta \neq \gamma$ in $\mathscr{B}$ so that $\left\|x_{i}^{\beta}-x_{i}^{\gamma}\right\|<\epsilon$ for $1 \leq i \leq n$. Let $K_{0} \in \mathcal{B}\left(\mathcal{H}_{\gamma}\right)$ be the restriction of $P_{\gamma} P_{\beta}$ to $\mathcal{H}_{\gamma}$. Then the following properties of $K_{0}$ are valid.

(i) $K_{0}$ is self-adjoint.

(ii) $K_{0}$ is compact. Indeed, $\left.P_{\beta}\right|_{\mathcal{H}_{\gamma}}$ intertwines $T_{\gamma}$ and $T_{\beta}$ and thus is compact by assumption. 
(iii) $K_{0}$ commutes with $T_{\gamma}$. Indeed, since $\mathcal{H}_{\beta}$ and $\mathcal{H}_{\gamma}$ are reducing subspaces for $T$, $P_{\beta}$ and $P_{\gamma}$ commute with $T$. Thus, $P_{\gamma} P_{\beta} T=T P_{\gamma} P_{\beta}$ and so $\left.\left.P_{\gamma} P_{\beta}\right|_{\mathcal{H}_{\gamma}} T\right|_{\mathcal{H}_{\gamma}}=$ $\left.\left.T\right|_{\mathcal{H}_{\gamma}} P_{\gamma} P_{\beta}\right|_{\mathcal{H}_{\gamma}}$. Hence, $K_{0} T_{\gamma}=T_{\gamma} K_{0}$.

(iv) $\left\|x_{i}^{\gamma}-K_{0} x_{i}^{\gamma}\right\|<\epsilon_{\gamma}$ for $1 \leq i \leq n$. Indeed,

$$
\begin{aligned}
\left\|x_{i}^{\gamma}-K_{0} x_{i}^{\gamma}\right\| & =\left\|x_{i}^{\gamma}-P_{\gamma} P_{\beta} x_{i}^{\gamma}\right\| \\
& =\left\|P_{\gamma} x_{i}^{\gamma}-P_{\gamma} P_{\beta} x_{i}^{\gamma}\right\| \\
& \leq\left\|\left(I-P_{\beta}\right) x_{i}^{\gamma}\right\|=\left\|\left(I-P_{\beta}\right)\left(x_{i}^{\gamma}-x_{i}^{\beta}\right)\right\| \leq\left\|x_{i}^{\gamma}-x_{i}^{\beta}\right\|<\epsilon \leq \epsilon_{\gamma} .
\end{aligned}
$$

These properties of $K_{0}$ contradict the choice of $\left(x_{i}^{\gamma}\right)_{i=1}^{n}$ and the proof is complete.

The replacements needed to transform Lemma 3.7 and its proof to Lemma 3.8 and its proof are 
Lemma 3.7

$E_{p}$

X

$Y_{p}$

$Q_{p}$

$\left(y_{i}^{p}\right)_{i=1}^{n(p)}$

$K: Y_{p} \rightarrow Y_{p}$ compact
Lemma 3.8

$S_{\alpha}$

T

$T_{\alpha}$

$P_{\alpha}$

$\left(x_{i}^{\alpha}\right)_{i=1}^{n(\alpha)}$

$K \in \mathcal{K}(\mathcal{H})$ commuting with $T_{\alpha}$,

i.e, $K \in \mathcal{K}(\mathcal{H})$ intertwining $T_{\alpha}$ and $T_{\alpha}$

$\mathscr{B}$

Also, $E_{p}$ failing the BCAP is replaced by $S_{\alpha}$ not being quasidiagonal. This is because $E_{p}$ failing the BCAP means that for every $M>0$,

$$
I \notin\left\{K: E_{p} \rightarrow E_{p}: K \text { is compact and }\|K\| \leq M\right\}^{-S O T}
$$

which, according to the Replacement Rule, could be replaced by

$$
I \notin\left\{K \in \mathcal{K}(\mathcal{H}): K S_{\alpha}=S_{\alpha} K \text { and }\|K\| \leq M\right\}^{-S O T}
$$

But in the context of operator theory, it is natural to add the condition that $K^{*}=K$, 
so $E_{p}$ failing the BCAP could be replaced by

$$
I \notin\left\{K \in \mathcal{K}(\mathcal{H}): K S_{\alpha}=S_{\alpha} K,\|K\| \leq M \text { and } K^{*}=K\right\}^{-S O T},
$$

which is equivalent to $S_{\alpha}$ not being block diagonal by Proposition 3.3. But BCAP preserves isomorphism whereas block diagonality does not preserve compalence. So $E_{p}$ failing the BCAP should be replaced by $S_{\alpha}$ not being quasidiagonal.

Proof of Theorem 3.5. That (ii) $\Rightarrow$ (i) follows easily from Theorem 3.4. To prove Not (ii) $\Rightarrow$ Not (i), suppose that (ii) is not true, i.e., there are uncountably many nonquasidiagonal operators in $\mathcal{C}$. By assumption and Lemma 3.2, every operator intertwining two different operators in $\mathcal{C}$ is compact. Thus, by Lemma 3.8, (i) is not true.

We conclude this subsection with two corollaries of Theorem 3.5. The first one is a direct consequence of Theorem 3.5 while the second one easily follows from the first one since a Fredholm operator that is quasidiagonal must have index 0 .

Corollary 3.9. Let $T_{0} \in \mathcal{B}(\mathcal{H})$ with $\sigma_{e}\left(T_{0}\right) \subset\{z \in \mathbb{C}:|z|=1\}$. Then $T_{0}$ is quasidiagonal if and only if there is an operator $T \in \mathcal{B}(\mathcal{H})$ such that for every $\alpha \in[0,1], \alpha T_{0}$ is compalent to a reducing part of $T$.

Corollary 3.10. Let $U$ be the unilateral shift. There is no operator $T \in \mathcal{B}(\mathcal{H})$ such that for every $\alpha \in[0,1], \alpha U$ is compalent to a reducing part of $T$. In particular, there is no operator $T \in \mathcal{B}(\mathcal{H})$ such that every contraction in $\mathcal{B}(\mathcal{H})$ is compalent to a reducing part of $T$.

\subsection{Ultraproducts of operators}

We begin by recalling from [38] a slight reformulation of the construction of the Calkin representation in the language of ultraproducts. 
Let $\mathscr{U}$ be a free ultrafilter on $\mathbb{N}$. If $\left(a_{n}\right)_{n \geq 1}$ is a bounded sequence in $\mathbb{C}$, then its ultralimit through $\mathscr{U}$ is denoted by $\lim _{n, \mathscr{U}} a_{n}$. Consider the Banach space

$$
\mathcal{H}^{\mathscr{U}}:=l_{\infty}(\mathcal{H}) /\left\{\left(x_{n}\right)_{n \geq 1} \in l_{\infty}(\mathcal{H}): \lim _{n, \mathscr{U}}\left\|x_{n}\right\|=0\right\}
$$

If $\left(x_{n}\right)_{n \geq 1} \in l_{\infty}(\mathcal{H})$ then its image in $\mathcal{H}^{\mathscr{U}}$ is denoted by $\left(x_{n}\right)_{\mathscr{U}}$, and it can be easily checked that

$$
\left\|\left(x_{n}\right)_{\mathscr{U}}\right\|=\lim _{n, \mathscr{U}}\left\|x_{n}\right\|
$$

Moreover, $\mathcal{H}^{\mathscr{U}}$ is, in fact, a Hilbert space with inner product

$$
\left\langle\left(x_{n}\right)_{\mathscr{U}},\left(y_{n}\right)_{\mathscr{U}}\right\rangle=\lim _{n, \mathscr{U}}\left\langle x_{n}, y_{n}\right\rangle
$$

But $\mathcal{H}^{\mathscr{U}}$ is nonseparable (see, e.g., [11, Proposition 8.5]).

If $\left(T_{n}\right)_{n \geq 1}$ is a bounded sequence in $\mathcal{B}(\mathcal{H})$, then its ultraproduct $\left(T_{1}, T_{2}, \ldots\right)_{\mathscr{U}} \in$ $\mathcal{B}\left(\mathcal{H}^{\mathscr{U}}\right)$ is defined by $\left(x_{n}\right)_{\mathscr{U}} \mapsto\left(T_{n} x_{n}\right)_{\mathscr{U}}$. If $T \in \mathcal{B}(\mathcal{H})$ then its ultrapower $T^{\mathscr{U}} \in$ $\mathcal{B}\left(\mathcal{H}^{\mathscr{U}}\right)$ is defined by $\left(x_{n}\right)_{\mathscr{U}} \mapsto\left(T x_{n}\right)_{\mathscr{U}}$. It is easy to see that

$$
\left(T_{1}, T_{2}, \ldots\right)_{\mathscr{U}}^{*}=\left(T_{1}^{*}, T_{2}^{*}, \ldots\right)_{\mathscr{U}}
$$

and in particular, $\left(T^{\mathscr{U}}\right)^{*}=\left(T^{*}\right)^{\mathscr{U}}$.

We pause here for a while to show that the strong limit of a sequence of normal operators on $\mathcal{H}$ is subnormal, using the ultraproduct construction. A stronger result was proven in [3, Theorem 3.3] and also in [9] where the strong limit of a net of normal operators on $\mathcal{H}$ was shown to be subnormal. Suppose that $\left(T_{n}\right)_{n \geq 1}$ is a sequence of normal operators on $\mathcal{H}$ converging in SOT to $T \in \mathcal{B}(\mathcal{H})$. The uniform boundedness principle gives $\sup _{n \geq 1}\left\|T_{n}\right\|<\infty$. Hence, the ultraproduct $\left(T_{1}, T_{2}, \ldots\right)_{\mathscr{U}}$ is well defined 
and is normal. Moreover, $\left\{(x)_{\mathscr{U}}: x \in \mathcal{H}\right\}$ is invariant under this operator, and $\left.T \cong\left(T_{1}, T_{2}, \ldots\right)_{\mathscr{U}}\right|_{\left\{(x)_{\mathscr{U}}: x \in \mathcal{H}\right\}}$. Therefore, $T$ is subnormal.

Consider the subspace

$$
\widehat{\mathcal{H}}:=\left\{\left(x_{n}\right)_{\mathscr{U}} \in \mathcal{H}^{\mathscr{U}}: w-\lim _{n, \mathscr{U}} x_{n}=0\right\}
$$

Here $w$ - $\lim _{n, \mathscr{U}} x_{n}$ is the weak limit of $\left(x_{n}\right)_{n \geq 1}$ through $\mathscr{U}$, i.e., the unique element $x \in \mathcal{H}$ such that

$$
\langle x, y\rangle=\lim _{n, \mathscr{U}}\left\langle x_{n}, y\right\rangle, \quad y \in \mathcal{H}
$$

Note that $\left\{(x)_{\mathscr{U}}: x \in \mathcal{H}\right\}^{\perp}=\widehat{\mathcal{H}}$, and thus,

$$
\widehat{\mathcal{H}}^{\perp}=\left\{(x)_{\mathscr{U}}: x \in \mathcal{H}\right\}
$$

The orthogonal projection from $\mathcal{H}^{\mathscr{U}}$ onto $\widehat{\mathcal{H}}^{\perp}$ is given by $\left(x_{n}\right)_{\mathscr{U}} \mapsto\left(w-\lim _{k, \mathscr{U}} x_{k}\right)_{\mathscr{U}}$. We shall identify the space $\widehat{\mathcal{H}}^{\perp}$ with $\mathcal{H}$ in the natural way. So we have $\mathcal{H}^{\mathscr{U}}=\mathcal{H} \oplus \widehat{\mathcal{H}}$.

For $T \in \mathcal{B}(\mathcal{H}), \widehat{\mathcal{H}}$ is a reducing subspace for $T^{\mathscr{U}}$ and define $\widehat{T} \in \mathcal{B}(\widehat{\mathcal{H}})$ by

$$
\widehat{T}:=\left.T^{\mathscr{U}}\right|_{\widehat{\mathcal{H}}}
$$

Thus, we have

$$
T^{\mathscr{U}}=T \oplus \widehat{T}
$$

with respect to the decomposition $\mathcal{H}^{\mathscr{U}}=\mathcal{H} \oplus \widehat{\mathcal{H}}$.

Note that $\widehat{K}=0$ for $K \in \mathcal{K}(\mathcal{H})$. (The proof uses the fact that every sequence in a compact metric space converges to an element through $\mathscr{U}$.) The map $f$ : $\mathcal{B}(\mathcal{H}) / \mathcal{K}(\mathcal{H}) \rightarrow \mathcal{B}(\widehat{\mathcal{H}})$ defined by $\pi(T) \mapsto \widehat{T}$ is the Calkin representation.

Theorem 3.11 ([8], Theorem 5.5). The map $f$ is an isometric *-isomorphism into 
$\mathcal{B}(\widehat{\mathcal{H}})$.

The following lemma will be useful throughout this subsection.

Lemma 3.12. Let $T_{1}, T_{3} \in \mathcal{B}(\mathcal{H})$ and let $T_{2}, T_{4}$ be operators on a (not necessarily separable) Hilbert space $\widetilde{\mathcal{H}}$. If $T_{1} \oplus T_{2} \cong T_{3} \oplus T_{4}$ then there is a separable reducing subspace $\mathcal{M}$ for both $T_{2}$ and $T_{4}$ such that

$$
T_{1} \oplus\left(\left.T_{2}\right|_{\mathcal{M}}\right) \cong T_{3} \oplus\left(\left.T_{4}\right|_{\mathcal{M}}\right)
$$

Proof. Let $W$ be a unitary operator on $\mathcal{H} \oplus \widetilde{\mathcal{H}}$ such that

$$
W\left(T_{1} \oplus T_{2}\right)=\left(T_{3} \oplus T_{4}\right) W .
$$

Let

$$
\mathcal{N}=\left\{S y: y \in \mathcal{H} \oplus\{0\} \text { and } S \in C^{*}\left(T_{1} \oplus T_{2}, T_{3} \oplus T_{4}, W\right)\right\}^{-\|\|},
$$

where $C^{*}\left(T_{1} \oplus T_{2}, T_{3} \oplus T_{4}, W\right)$ is the unital $C^{*}$-subalgebra of $\mathcal{B}(\mathcal{H} \oplus \widetilde{\mathcal{H}})$ generated by $T_{1} \oplus T_{2}, T_{3} \oplus T_{4}$ and $W$. Then $\mathcal{N}$ is a separable reducing subspace for $T_{1} \oplus T_{2}$, $T_{3} \oplus T_{4}$ and $W$. Since $\mathcal{H} \oplus\{0\} \subset \mathcal{N}$, there exists a subspace $\mathcal{M} \subset \widetilde{\mathcal{H}}$ such that $\mathcal{N}=\mathcal{H} \oplus \mathcal{M}$, and thus $\mathcal{M}$ is a separable reducing subspace for $T_{2}$ and $T_{4}$. Moreover, since $\mathcal{N}$ reduces $W,\left.W\right|_{\mathcal{N}}$ is a unitary operator on $\mathcal{N}$ and satisfies

$$
\left(\left.W\right|_{\mathcal{N}}\right)\left(T_{1} \oplus\left(\left.T_{2}\right|_{\mathcal{M}}\right)\right)=\left(T_{3} \oplus\left(\left.T_{4}\right|_{\mathcal{M}}\right)\right)\left(\left.W\right|_{\mathcal{N}}\right) .
$$

Therefore,

$$
T_{1} \oplus\left(\left.T_{2}\right|_{\mathcal{M}}\right) \cong T_{3} \oplus\left(\left.T_{4}\right|_{\mathcal{M}}\right)
$$


The Calkin representation yields an alternative proof of the following known result (see, e.g., [36, Theorem 2.29]):

If $T, K \in \mathcal{K}(\mathcal{H})$ and $T \simeq_{a} K$ then $T \oplus 0_{\mathcal{H}} \cong K \oplus 0_{\mathcal{H}}$, where $0_{\mathcal{H}}$ is the zero operator on $\mathcal{H}$.

Since $T \simeq_{a} K$, there exists a sequence $\left(W_{n}\right)_{n \geq 1}$ of unitary operators on $\mathcal{H}$ such that $\lim _{n \rightarrow \infty}\left\|T-W_{n} K W_{n}^{*}\right\|=0$. Thus,

$$
T^{\mathscr{U}}=\left(W_{1} K W_{1}^{*}, W_{2} K W_{2}^{*}, \ldots\right)_{\mathscr{U}}=\left(W_{1}, W_{2}, \ldots\right)_{\mathscr{U}} K^{\mathscr{U}}\left(W_{1}, W_{2}, \ldots\right)_{\mathscr{U}}^{*}
$$

and so $T^{\mathscr{U}} \cong K^{\mathscr{U}}$. Since $T, K \in \mathcal{K}(\mathcal{H})$, this implies that

$$
T \oplus 0_{\widehat{\mathcal{H}}}=T \oplus \widehat{T}=T^{\mathscr{U}} \cong K^{\mathscr{U}}=K \oplus \widehat{K}=K \oplus 0_{\widehat{\mathcal{H}}}
$$

By Lemma 3.12, $T \oplus 0_{\mathcal{H}} \cong K \oplus 0_{\mathcal{H}}$.

Let us recall a result of Voiculescu.

Theorem 3.13 ([41], Theorem 1.3). Let $T \in \mathcal{B}(\mathcal{H})$ and let $\rho$ be a unital representation of $C^{*}(\pi(T))$ on a separable Hilbert space $\mathcal{H}_{\rho}$. Then $T \simeq_{a} T \oplus \rho(\pi(T))$.

If $T \in \mathcal{B}(\mathcal{H})$ and $\mathcal{M}$ is a separable reducing subspace for $\widehat{T}$, then $\left.\pi(S) \rightarrow \widehat{S}\right|_{M}$ defines a unital representation of $C^{*}(\pi(T))$ on $\mathcal{M}$. Applying Theorem 3.13 to this representation, we obtain

Theorem 3.14. Let $T \in \mathcal{B}(\mathcal{H})$ and let $\mathcal{M}$ be a separable reducing subspace for $\widehat{T}$. Then $T \simeq_{a} T \oplus\left(\left.\widehat{T}\right|_{\mathcal{M}}\right)$.

Theorem 3.15. If $T_{1}, T_{2} \in \mathcal{B}(\mathcal{H})$ then $T_{1} \simeq{ }_{a} T_{2}$ if and only if $T_{1}^{\mathscr{U}} \cong T_{2}^{\mathscr{U}}$.

Proof. If $T_{1} \simeq_{a} T_{2}$ then from a similar argument as in the discussion preceding Theorem 3.13, we have $T_{1}^{\mathscr{U}} \cong T_{2}^{\mathscr{U}}$. Conversely, suppose that $T_{1}^{\mathscr{U}} \cong T_{2}^{\mathscr{U}}$. Then 
$T_{1} \oplus \widehat{T}_{1} \cong T_{2} \oplus \widehat{T}_{2}$, and thus by Lemma 3.12 , there exists a separable reducing subspace $\mathcal{M}$ for both $\widehat{T}_{1}$ and $\widehat{T}_{2}$ such that

$$
T_{1} \oplus\left(\left.\widehat{T}_{1}\right|_{\mathcal{M}}\right) \cong T_{2} \oplus\left(\left.\widehat{T}_{2}\right|_{\mathcal{M}}\right)
$$

Thus, by Theorem 3.14, we obtain $T_{1} \simeq_{a} T_{2}$.

Although we will not make use of Theorem 3.15, the proofs of the results below resemble the proof of this theorem.

Let $\mathcal{H}_{1}$ and $\mathcal{H}_{2}$ be Hilbert spaces and let $\lambda \geq 1$. Then two operators $T_{1} \in \mathcal{B}\left(\mathcal{H}_{1}\right)$ and $T_{2} \in \mathcal{B}\left(\mathcal{H}_{2}\right)$ are $\lambda$-similar if there is an invertible operator $S \in \mathcal{B}\left(\mathcal{H}_{1}, \mathcal{H}_{2}\right)$ such that $T_{2}=S T_{1} S^{-1}$ and $\|S\|\left\|S^{-1}\right\| \leq \lambda$.

Theorem 3.16. Suppose that $T_{1}, T_{2} \in \mathcal{B}(\mathcal{H})$. If $\lambda \geq 1$ and

$$
T_{2} \in\left\{S T_{1} S^{-1}: S \in \mathcal{B}(\mathcal{H}) \text { with }\|S\|\left\|S^{-1}\right\| \leq \lambda\right\}^{-\|\|}
$$

then there exists a sequence $\left(S_{n}\right)_{n \geq 1}$ of invertible operators on $\mathcal{H}$ with $\left\|S_{n}\right\|\left\|S_{n}^{-1}\right\| \leq \lambda$ such that $\lim _{n \rightarrow \infty}\left\|T_{2}-S_{n} T_{1} S_{n}^{-1}\right\|=0$ and $T_{2}-S_{n} T_{1} S_{n}^{-1} \in \mathcal{K}(\mathcal{H})$.

Proof. Let $\left(R_{n}\right)_{n \geq 1}$ be a sequence in $\mathcal{B}(\mathcal{H})$ with $\left\|R_{n}\right\|\left\|R_{n}^{-1}\right\| \leq \lambda$ such that $\lim _{n \rightarrow \infty} \| T_{2}-$ $R_{n} T_{1} R_{n}^{-1} \|=0$. Without loss of generality, we may assume that $\left\|R_{n}\right\| \leq \lambda$ and $\left\|R_{n}^{-1}\right\| \leq 1$, so that $\sup _{n \geq 1}\left\|R_{n}\right\|, \sup _{n \geq 1}\left\|R_{n}^{-1}\right\|<\infty$. Then

$$
\begin{aligned}
T_{2}^{\mathscr{U}} & =\left(R_{1} T_{1} R_{1}^{-1}, R_{2} T_{1} R_{2}^{-1}, R_{3} T_{1} R_{3}^{-1}, \ldots\right)_{\mathscr{U}} \\
& =\left(R_{1}, R_{2}, R_{3}, \ldots\right)_{\mathscr{U}} T_{1}^{\mathscr{U}}\left(R_{1}, R_{2}, R_{3}, \ldots\right)_{\mathscr{U}}^{-1} .
\end{aligned}
$$

Hence, $T_{1}^{\mathscr{U}}$ is $\lambda$-similar to $T_{2}^{\mathscr{U}}$, and so $T_{1} \oplus \widehat{T}_{1}$ is $\lambda$-similar to $T_{2} \oplus \widehat{T}_{2}$. By a variation of Lemma 3.12 , there exists a separable reducing subspace $\mathcal{M}$ for both $\widehat{T}_{1}$ and $\widehat{T}_{2}$ such 
that $T_{1} \oplus\left(\left.\widehat{T}_{1}\right|_{\mathcal{M}}\right)$ is $\lambda$-similar to $T_{2} \oplus\left(\left.\widehat{T}_{2}\right|_{\mathcal{M}}\right)$. By Theorem 3.14, the result follows.

The preceding theorem was proven in [41] for $\lambda=1$ (i.e., $T_{2} \in \mathcal{U}\left(T_{1}\right)^{-\|\|} \Rightarrow T_{1} \simeq_{a}$ $T_{2}$ ) by applying Theorem 3.13 in a different way.

The rest of this subsection is mainly devoted to proving Theorem 3.17 below.

In the sequel, when we say that an operator $T_{1} \in \mathcal{B}(\mathcal{H})$ is a restriction of another operator $T_{2} \in \mathcal{B}(\mathcal{H})$, we mean that $T_{1}$ is unitarily equivalent to a restriction of $T_{2}$. We do the same thing for compression and reducing part. This is to simplify our presentation.

Theorem 3.17 ([18], Theorem 4.3 and [19], Theorem 4.4). Let $T \in \mathcal{B}(\mathcal{H})$. Then

$$
\begin{aligned}
& \mathcal{U}(T)^{-S O T}=\left\{B \in \mathcal{B}(\mathcal{H}): B \text { is a restriction of an operator in } \mathcal{U}(T)^{-\|\|}\right\}, \\
& \mathcal{U}(T)^{-W O T}=\left\{B \in \mathcal{B}(\mathcal{H}): B \text { is a compression of an operator in } \mathcal{U}(T)^{-\|\|}\right\}, \\
& \mathcal{U}(T)^{-*-S O T}=\left\{B \in \mathcal{B}(\mathcal{H}): B \text { is a reducing part of an operator in } \mathcal{U}(T)^{-\|\|}\right\} .
\end{aligned}
$$

The idea of this result is the following lemma.

Lemma 3.18. Let $\left(T_{n}\right)_{n \geq 1}$ be a sequence in $\mathcal{B}(\mathcal{H})$ and let $B \in \mathcal{B}(\mathcal{H})$.

(1) If $T_{n} \rightarrow B$ in SOT then $B$ is a restriction of $\left(T_{1}, T_{2}, \ldots\right)_{\mathscr{U}}$.

(2) If $T_{n} \rightarrow B$ in WOT then $B$ is a compression of $\left(T_{1}, T_{2}, \ldots\right)_{\mathscr{U}}$.

(3) If $T_{n} \rightarrow B$ in $*-S O T$ then $B$ is a redcing part of $\left(T_{1}, T_{2}, \ldots\right)_{\mathscr{U}}$.

Proof. By the uniform boundedness principle, $\sup _{n \geq 1}\left\|T_{n}\right\|<\infty$, so the ultraproduct $\left(T_{1}, T_{2}, \ldots\right)_{\mathscr{U}}$ is well defined.

If $T_{n} \rightarrow B$ in SOT then $\left\{(x)_{\mathscr{U}}: x \in \mathcal{H}\right\}$ is invariant under $\left(T_{1}, T_{2}, \ldots\right)_{\mathscr{U}}$, and $\left.B \cong\left(T_{1}, T_{2}, \ldots\right){ }_{\mathscr{U}}\right|_{\left\{(x)_{\mathscr{U}}: x \in \mathcal{H}\right\}}$. 
Suppose that $T_{n} \rightarrow B$ in WOT. Recall that the orthogonal projection from $\mathcal{H}^{\mathscr{U}}$ onto $\left\{(x)_{\mathscr{U}}: x \in \mathcal{H}\right\}$ is given by $\left(x_{n}\right)_{\mathscr{U}} \mapsto\left(w-\lim _{k, \mathscr{U}} x_{k}\right)_{\mathscr{U}}$. Thus, the compression of $\left(T_{1}, T_{2}, \ldots\right)_{\mathscr{U}}$ to $\left\{(x)_{\mathscr{U}}: x \in \mathcal{H}\right\}$ is given by $(x)_{\mathscr{U}} \mapsto\left(w-\lim _{k, \mathscr{U}} T_{k} x\right)_{\mathscr{U}}=(B x)_{\mathscr{U}}$. Hence, $B$ is a compression of $\left(T_{1}, T_{2}, \ldots\right)_{\mathscr{U}}$.

If $T_{n} \rightarrow B$ in $*$-SOT then $\left\{(x)_{\mathscr{U}}: x \in \mathcal{H}\right\}$ is a reducing subspace for $\left(T_{1}, T_{2}, \ldots\right)_{\mathscr{U}}$, and $\left.B \cong\left(T_{1}, T_{2}, \ldots\right)_{\mathscr{U}}\right|_{\left\{(x)_{\mathscr{U}}: x \in \mathcal{H}\right\}}$.

Proof of Theorem 3.17. If $B \in \mathcal{U}(T)^{-S O T}$ then there exists a sequence $\left(W_{n}\right)_{n \geq 1}$ of unitary operators in $\mathcal{B}(\mathcal{H})$ such that $W_{n} T W_{n}^{*} \rightarrow B$ in SOT. Thus, by Lemma 3.18, $B$ is a restriction of $\left(W_{1} T W_{1}^{*}, W_{2} T W_{2}^{*}, \ldots\right)_{\mathscr{U}} \cong T^{\mathscr{U}} \cong T \oplus \widehat{T}$. Hence, there exists a separable reducing subspace $\mathcal{M}$ for $\widehat{T}$ such that $B$ is a restriction of $T \oplus\left(\left.\widehat{T}\right|_{\mathcal{M}}\right)$. But by Theorem 3.14, $T \oplus\left(\left.\widehat{T}\right|_{\mathcal{M}}\right) \simeq_{a} T$. Therefore, one inclusion of (3.2) is proven.

The proof of the other inclusion here is more or less the same as that in [19], but we include it here for self-containedness. To prove this inclusion, it suffices to show that if $B$ is a restriction of $T$, then $B \in \mathcal{U}(T)^{-S O T}$. This is an immediate consequence of the next lemma. Thus, the proof of (3.2) is complete.

The proofs of the other assertions are similar using some variations of the next lemma.

Lemma 3.19. Suppose that $T \in \mathcal{B}(\mathcal{H} \oplus \mathcal{H})$ and that $\mathcal{H} \oplus\{0\}$ is an invariant subspace for $T$. Let $B=\left.T\right|_{\mathcal{H} \oplus\{0\}} \in \mathcal{B}(\mathcal{H} \oplus\{0\})$. Then there exists a sequence $\left(W_{n}\right)_{n \geq 1}$ of unitary operators in $\mathcal{B}(\mathcal{H} \oplus \mathcal{H}, \mathcal{H} \oplus\{0\})$ such that $W_{n} T W_{n}^{*} \rightarrow B$ in SOT.

Proof. Let $P_{n}$ be a sequence of finite-rank orthogonal projections converging in SOT to the identity operator on $\mathcal{H}$. Let $W_{n}: \mathcal{H} \oplus \mathcal{H} \rightarrow \mathcal{H} \oplus\{0\}$ be a unitary operator such that

$$
W_{n}(x, 0)=(x, 0), \quad x \in P_{n} \mathcal{H}
$$


Then

$$
W_{n}\left[\left(I-P_{n}\right) \mathcal{H} \oplus \mathcal{H}\right]=\left(I-P_{n}\right) \mathcal{H} \oplus\{0\}
$$

For $x \in P_{n} \mathcal{H}$,

$$
\begin{aligned}
\left(B-W_{n} T W_{n}^{*}\right)(x, 0)= & B(x, 0)-W_{n} T(x, 0) \\
= & B(x, 0)-W_{n} B(x, 0) \\
= & B(x, 0)-W_{n}\left(P_{n} \oplus 0\right) B(x, 0) \\
& -W_{n}\left(\left(I-P_{n}\right) \oplus 0\right) B(x, 0) \\
= & B(x, 0)-\left(P_{n} \oplus 0\right) B(x, 0) \\
& -W_{n}\left(\left(I-P_{n}\right) \oplus 0\right) B(x, 0) \\
= & \left(\left(I-P_{n}\right) \oplus 0\right) B(x, 0)-W_{n}\left(\left(I-P_{n}\right) \oplus 0\right) B(x, 0),
\end{aligned}
$$

and thus,

$$
\left\|\left(B-W_{n} T W_{n}^{*}\right)(x, 0)\right\| \leq 2\left\|\left(\left(I-P_{n}\right) \oplus 0\right) B(x, 0)\right\|, \quad x \in P_{n} \mathcal{H} .
$$


Hence, for $x \in \mathcal{H}$,

$$
\begin{aligned}
\left\|\left(B-W_{n} T W_{n}^{*}\right)(x, 0)\right\| \leq & \left\|\left(B-W_{n} T W_{n}^{*}\right)\left(P_{n} x, 0\right)\right\| \\
& +\left\|\left(B-W_{n} T W_{n}^{*}\right)\left(\left(I-P_{n}\right) x, 0\right)\right\| \\
\leq & 2\left\|\left(\left(I-P_{n}\right) \oplus 0\right) B\left(P_{n} x, 0\right)\right\| \\
& +\left\|B-W_{n} T W_{n}^{*}\right\|\left\|\left(I-P_{n}\right) x\right\| \\
\leq & 2\left\|\left(\left(I-P_{n}\right) \oplus 0\right) B(x, 0)\right\| \\
& +2\left\|\left(\left(I-P_{n}\right) \oplus 0\right) B\left(\left(I-P_{n}\right) x, 0\right)\right\| \\
& +\left\|B-W_{n} T W_{n}^{*}\right\|\left\|\left(I-P_{n}\right) x\right\| \\
\leq & 2\left\|\left(\left(I-P_{n}\right) \oplus 0\right) B(x, 0)\right\| \\
& +2\left\|\left(\left(I-P_{n}\right) \oplus 0\right) B\left(\left(I-P_{n}\right) x, 0\right)\right\| \\
& +(\|B\|+\|T\|)\left\|\left(I-P_{n}\right) x\right\| \\
\leq & 2\left\|\left(\left(I-P_{n}\right) \oplus 0\right) B(x, 0)\right\|+2\|B\|\left\|\left(I-P_{n}\right) x\right\| \\
& +(\|B\|+\|T\|)\left\|\left(I-P_{n}\right) x\right\| \rightarrow 0,
\end{aligned}
$$

as $n \rightarrow \infty$. Therefore, $W_{n} T W_{n}^{*} \rightarrow B$ in SOT.

The following result seems to be known. (The results in [10] are somewhat related to this result.)

Theorem 3.20. Let $T_{1}, T_{2} \in \mathcal{B}(\mathcal{H})$. Suppose that there is a sequence $\left(P_{n}\right)_{n \geq 1}$ of finite rank orthogonal projections on $\mathcal{H}$ such that $P_{n} \rightarrow I$ in $S O T$ and $\left.P_{n} T_{1}\right|_{P_{n} \mathcal{H}}$ is a restriction (resp. compression, reducing part) of $T_{2}$. Then $T_{1}$ is a restriction (resp. compression, reducing part) of an operator in $\mathcal{U}\left(T_{2}\right)^{-\|\|}$.

Proof. The operator $T_{1}$ is a reducing part of $\left(\left.P_{1} T_{1}\right|_{P_{1} \mathcal{H}},\left.P_{2} T_{1}\right|_{P_{2} \mathcal{H}},\left.P_{3} T_{1}\right|_{P_{3} \mathcal{H}}\right) \mathscr{U}$ via the map $x \mapsto\left(P_{n} x\right)_{\mathscr{U}}$. Hence, by assumption, $T_{1}$ is a restriction of $T_{2}^{\mathscr{U}} \cong T_{2} \oplus \widehat{T}_{2}$. Then 
we can find a separable reducing subspace $\mathcal{M}$ for $\widehat{T}_{2}$ such that $T_{1}$ is a restriction of $T_{2} \oplus\left(\left.\widehat{T}_{2}\right|_{\mathcal{M}}\right)$. But by Theorem 3.13, $T_{2} \oplus\left(\left.\widehat{T}_{2}\right|_{\mathcal{M}}\right) \simeq_{a} T_{2}$. Thus, the result follows.

We conclude by briefly explaining how the work in this subsection was derived. Suppose that the Banach spaces $X_{1}, X_{2}, \ldots$ have been replaced by operators $T_{1}, T_{2}, \ldots \in \mathcal{B}(\mathcal{H})$, respectively. This suggests to replace the ultraproduct $\left(X_{1}, X_{2}, \ldots\right)_{\mathscr{U}}$ by the operator $\left(T_{1}, T_{2}, \ldots\right)_{\mathscr{U}}$. In other words, the ultraproduct of Banach spaces should be replaced by the ultraproduct of the corresponding operators. The preceding result was motivated by the concept of finite representability of Banach spaces (see, e.g., [11, Chapter 8]), which is closely related to ultraproducts of Banach spaces. The other results Theorem 3.15 and Theorem 3.16 and the proof of Theorem 3.17 were inspired by the proof of the preceding result. 


\section{ASYMPTOTIC $*$-MOMENTS OF SOME RANDOM VANDERMONDE MATRICES}

This section comes from [6].

\subsection{Introduction}

We consider the random Vandermonde matrix $X_{N}$, whose $(i, j)$-th entry is $N^{-1 / 2} \zeta_{i}^{j}$, where $\zeta_{1}, \ldots, \zeta_{N}$ are independent with Haar measure distribution on the unit circle. These have been studied in [30], [31], [34] and [35] and are of interest for applications in finance, signal array processing, wireless communications and biology (see [30] for references). In [30], Ryan and Debbah show that asymptotic moments of $X_{N}^{*} X_{N}$, (namely, the limits

$$
\lim _{N \rightarrow \infty} \mathbb{E} \circ \operatorname{tr}\left(\left(X_{N}^{*} X_{N}\right)^{p}\right)
$$

where $\mathbb{E}$ is the expectation and tr is the normalized trace on matrix algebras), exist and are given by sums of volumes of certain polytopes. They also compute some of these asymptotic moments. In [34], Tucci and Whiting show among other things that the asymptotic moments are given by

$$
\lim _{N \rightarrow \infty} \mathbb{E} \circ \operatorname{tr}\left(\left(X_{N}^{*} X_{N}\right)^{p}\right)=\int x^{p} d \mu(x)
$$

for a unique measure $\mu$ on $[0, \infty)$ with unbounded support. (This uses the Stieltjes solution to the moment problem and a theorem of Carleman - for the former, see p. 76 of [1].) Further results are proven in [31] and [35].

G. Tucci asked [33] whether $X_{N}$ is asymptotically R-diagonal with respect to the 
expectation of the trace. In this paper, we answer Tucci's question negatively, but show that $X_{N}$ has an asymptotic $*$-distribution as $N \rightarrow \infty$, which is in fact the *-distribution of an element that is $\mathrm{R}$-diagonal over the $\mathrm{C}^{*}$-algebra $C[0,1]$.

To be precise, we show that, for all $n \in \mathbf{N}$ and all $\epsilon(1), \ldots, \epsilon(n) \in\{1, *\}$,

$$
\lim _{N \rightarrow \infty} \mathbb{E} \circ \operatorname{tr}\left(X_{N}^{\epsilon(1)} \cdots X_{N}^{\epsilon(n)}\right)
$$

exists and we describe this limiting *-moment using the notion of $C[0,1]$-valued R-diagonality.

Usual (or scalar-valued) R-diagonal elements are very natural in free probability theory, and have been much studied; they were introduced by Nica and Speicher in [29]. The algebra-valued version was introduced by Śniady and Speicher in [32] and has been further studied in [7]. We will give the definition from [7], which is an easy reformulation of one of the characterizations in [32].

The setting for algebra-valued R-diagonal elements is a $B$-valued *-noncommutative probability space $(A, \mathcal{E})$, where $B \subseteq A$ is a unital inclusion of unital $*$-algebras and $\mathcal{E}: A \rightarrow B$ is a conditional expectation, namely, a $B$-bimodular unital projection.

Definition 4.1.1. Given $n \in \mathbf{N}$ and $\epsilon=(\epsilon(1), \ldots, \epsilon(n)) \in\{1, *\}^{n}$, we define the maximal alternating interval partition $\sigma(\epsilon)$ to be the interval partition of $\{1, \ldots, n\}$ whose blocks are the maximal interval subsets $I$ of $\{1, \ldots, n\}$ such that if $j \in I$ and $j+1 \in I$, then $\epsilon(j) \neq \epsilon(j+1)$.

For example, if $\epsilon=\{1,1, *, 1, *, *\}$, then $\sigma(\epsilon)=\{\{1\},\{2,3,4,5\},\{6\}\}$.

Definition 4.1.2. An element $a \in A$ is $B$-valued $R$-diagonal if for every integer 
$k \geq 0$ and every $b_{1}, \ldots, b_{2 k} \in B$ we have

$$
\mathcal{E}\left(a b_{1} a^{*} b_{2} a b_{3} a^{*} \cdots b_{2 k-2} a b_{2 k-1} a^{*} b_{2 k} a\right)=0
$$

(namely, odd alternating moments vanish) and, for every integer $n \geq 1$, every $\epsilon \in$ $\{1, *\}^{n}$ and every choice of $b_{1}, b_{2}, \ldots b_{n} \in B$, we have

$$
\mathcal{E}\left(\prod_{I \in \sigma(\epsilon)}\left(\left(\prod_{j \in I} b_{j} a^{\epsilon(j)}\right)-\mathcal{E}\left(\prod_{j \in I} b_{j} a^{\epsilon(j)}\right)\right)\right)=0
$$

where in each of the three products above, the terms are taken in order of increasing indices.

Note that the $B$-valued R-diagonality condition determines all of the $B$-valued *-moments

$$
\mathcal{E}\left(a^{\epsilon(1)} b_{1} a^{\epsilon(2)} \cdots b_{n-1} a^{\epsilon(n)}\right)
$$

for $n \in \mathbf{N}, b_{1}, \ldots, b_{n-1} \in B$ and arbitrary $\epsilon(1), \ldots, \epsilon(n) \in\{1, *\}$, in terms of the alternating moments of even length, namely those when $n$ is even and $\epsilon(j) \neq \epsilon(j+1)$ for all $j$.

Contents: The contents of the rest of the section are as follows. In Subsection 4.2, we find asymptotics of diagonal entries of $*$-moments involving alternating $X_{N}$ and $X_{N}^{*}$ with certain deterministic diagonal matrices between. In Subsection 4.3, we prove our main result which characterizes arbitrary asymptotic $*$-moments of $X_{N}$ based on $C[0,1]$-valued R-diagonality. In Subsection 4.4, we prove results allowing the asymptotic alternating $*$-moments of $X_{N}$ found in Subsection 4.2 to be computed in terms of certain integrals and we show that $X_{N}$ is not asymptotically scalar-valued R-diagonal. 
Notation: On matrix algebras, $\operatorname{tr}$ is the normalized trace and $\mathrm{Tr}$ is the usual trace. For partitions $\pi_{1}$ and $\pi_{2}$ of the same set, $\pi_{1} \vee \pi_{2}$ denotes their join in the lattice of all partitions of the set. We say that a set $S$ splits a partition $\pi$ if $S$ is the union of some of the blocks of $\pi$. We write $k_{1} \stackrel{\pi}{\sim} k_{2}$ to mean that $k_{1}$ and $k_{2}$ are in the same block of $\pi$. The restriction of a partition $\pi$ to a set $K$ is the partition $\{S \cap K: S \in \pi\} \backslash\{\emptyset\}$, and is denoted $\pi\left\lceil_{K}\right.$. If $i$ is a function with domain $L$, then ker $i$ is the partition of $L$ such that $\ell_{1}$ and $\ell_{2}$ belong to the same block of ker $i$ if and only if $i\left(\ell_{1}\right)=i\left(\ell_{2}\right)$.

\subsection{Asymptotic alternating $C[0,1]$-valued $*$-moments.}

In this subsection, we investigate alternating moments in $X_{N}$ and $X_{N}^{*}$. More specifically, we find the asymptotics of the expectations of diagonal elements of alternating moments of even length, with certain non-random diagonal matrices interspersed (see Proposition 4.1).

Let $\tau$ be the tracial state on $C[0,1]$ given by integration with Lebesgue measure.

Given $n \in \mathbf{N}$, we let $\mathcal{P}(n)$ denote the lattice of all set partitions of $\{1,2, \ldots, n\}$. Thus $\pi \in \mathcal{P}(n)$ if and only if $\pi$ is a collection of disjoint, nonempty sets whose union is $\{1, \ldots, n\}$. As usual, the elements of $\pi$ are called blocks of the partition, and $|\pi|$ is the number of blocks in the partition. We will let $S_{\pi}(j)$ denote the block of $\pi$ that has $j$ as an element.

For $\pi \in \mathcal{P}(n)$ and $g_{1}, \ldots g_{n} \in C[0,1]$, let $\Gamma_{\pi}\left(g_{1}, \ldots, g_{n}\right) \in C[0,1]$ be defined by

$$
\Gamma_{\pi}\left(g_{1}, \ldots, g_{n}\right)=\prod_{j \in S_{\pi}(1)} g_{j} \prod_{S \in \pi \backslash\left\{S_{\pi}(1)\right\}} \tau\left(\prod_{j \in S} g_{j}\right)
$$

Thus,

$$
\tau\left(\Gamma_{\pi}\left(g_{1}, \ldots, g_{n}\right)\right)=\prod_{S \in \pi} \tau\left(\prod_{j \in S} g_{j}\right)
$$

Given $S \in \pi$, we let $S^{\prime}=S \backslash\{\max (S)\}$ be $S$ without its largest element and we 
let

$$
J=J_{\pi}=\bigcup_{S \in \pi} S^{\prime} .
$$

Thus $|J|=n-|\pi|$. Naturally, we write $S_{\pi}^{\prime}(j)$ for $\left(S_{\pi}(j)\right)^{\prime}$. For $p \in\{1, \ldots, n\}$ and $S \in \pi$, we write $S \leq p$ if and only if $j \leq p$ for every $j \in S$, and if this is not the case, then we write $S \not \leq p$. Then

$$
I_{\pi}(p):=\left\{j \in\{1, \ldots, p\}: S_{\pi}(j) \not \leq p\right\} \subseteq J .
$$

If $J=\emptyset$, namely, if $\pi=0_{n}$ is the partition of $\{1, \ldots, n\}$ into singletons, then we let

$$
\Lambda_{\pi}\left(g_{1}, \ldots, g_{n-1}\right)=\prod_{j=1}^{n-1} g_{j},
$$

where if $n=1$ then we let $\Lambda_{\pi}()=1$ be the constant function 1 . Otherwise, if $J \neq \emptyset$, then for $t \in[0,1]$ we let

$$
E(\pi, t)=\left\{\left(t_{j}\right)_{j \in J} \in \mathbf{R}^{|J|} \mid \forall p \in\{1, \ldots, n-1\}, 0 \leq t+\sum_{j \in I_{\pi}(p)} t_{j} \leq 1\right\},
$$

and we set

$$
\Lambda_{\pi}\left(g_{1}, \ldots, g_{n-1}\right)(t)=\int_{E(\pi, t)}\left(\prod_{p=1}^{n-1} g_{p}\left(t+\sum_{j \in I_{\pi}(p)} t_{j}\right)\right) d \lambda\left(\left(t_{j}\right)_{j \in J}\right)
$$

where the integral is with respect to $|J|$-dimensional Lebesgue measure.

Remark. Here is an alternative description of $\Lambda_{\pi}$ that is more natural. Assume 
$\pi \in \mathcal{P}(n) \backslash\left\{0_{n}\right\}$ and let $\Phi: \mathbf{R}^{n-|\pi|} \times \mathbf{R} \rightarrow \mathbf{R}^{n}$ be the linear mapping given by

$$
\Phi\left(\left(t_{j}\right)_{j \in J_{\pi}}, t\right)=\left(t+\sum_{j \in I_{\pi}(p)} t_{j}\right)_{p=1}^{n}
$$

Then $\Phi$ is injective and is onto the $(n-|\pi|+1)$-dimensional subspace

$$
K_{\pi}=\left\{\left(s_{p}\right)_{p=1}^{n} \mid \forall S \in \pi, \sum_{p \in S} s_{p}-s_{p-1}=0\right\}
$$

of $\mathbf{R}^{n}$, using the convention $s_{0}=s_{n}$. Indeed, $\Phi$ is injective because if $\Phi\left(\left(t_{j}\right)_{j \in J_{\pi}}, t\right)=$ $\left(s_{p}\right)_{p=1}^{n}$, then $s_{n}=t$ and if $p \in J_{\pi}$, then $s_{p}$ equals $t_{p}$ plus $s_{n}$ plus some from the list $\left(t_{j}\right)_{j \in J_{\pi}, j \leq p-1}$. We see that $\Phi$ maps into $K_{\pi}$ after observing

$$
s_{p}-s_{p-1}= \begin{cases}t_{p}, & p \neq \max S_{\pi}(p) \\ -\sum_{i \in S_{\pi}(p) \backslash\{p\}} t_{i}, & p=\max S_{\pi}(p),\end{cases}
$$

and it is onto $K_{\pi}$ by a dimension count. Note that the push-forward under $\Phi$ of Lebesgue measure is the $(n-|\pi|+1)$-dimensional Lebesgue measure on $K_{\pi}$ corresponding to the restriction of the usual Euclidean metric of $\mathbf{R}^{n}$. We consider the $(n-|\pi|)$-dimensional affine space

$$
K_{\pi}^{(t)}=\left\{\left(s_{p}\right)_{p=1}^{n} \in K_{\pi}: s_{n}=t\right\}
$$

and we let $\nu^{(\pi, t)}$ be the $(n-|\pi|)$-dimensional Lebesgue measure on $K_{\pi}^{(t)}$ corresponding to the restriction to $K_{\pi}^{(t)}$ of the usual Euclidean metric on $\mathbf{R}^{n}$. Then $\Phi$ sends $E(\pi, t) \times\{t\}$ onto

$$
F(\pi, t):=\left\{\left(s_{p}\right)_{p=1}^{n} \in K_{\pi} \cap[0,1]^{n}: s_{n}=t\right\}
$$


and $\Lambda_{\pi}\left(g_{1}, \ldots, g_{n-1}\right)(t)$ is the integral of $g_{1} \otimes \cdots \otimes g_{n-1} \otimes 1$ over $F(\pi, t)$ with respect to $\nu^{(\pi, t)}$. Namely, we have

$$
\Lambda_{\pi}\left(g_{1}, \ldots, g_{n-1}\right)(t)=\int_{F(\pi, t)}\left(g_{1} \otimes \cdots \otimes g_{n-1} \otimes 1\right) d \nu^{(\pi, t)}
$$

Proposition 4.1. Let $n \in \mathbf{N}$ and suppose $g_{1}, \ldots, g_{2 n} \in C[0,1]$. Given $N \in \mathbf{N}$ and $j \in\{1, \ldots, 2 n\}$ consider the deterministic $N \times N$ diagonal matrix

$$
D_{N}^{(j)}=\operatorname{diag}\left(g_{j}\left(\frac{1}{N}\right), g_{j}\left(\frac{2}{N}\right), \ldots, g_{j}\left(\frac{N}{N}\right)\right)
$$

For $t \in[0,1]$, let $h_{N}(t)$ be the least element of $\{1, \ldots, N\}$ so that $t \leq h_{N}(t) / N$. Then for all $t \in[0,1]$,

$$
\begin{aligned}
\lim _{N \rightarrow \infty} \mathbb{E}\left(D_{N}^{(1)} X_{N}^{*} D_{N}^{(2)} X_{N} D_{N}^{(3)} X_{N}^{*} D_{N}^{(4)} X_{N} \cdots D_{N}^{(2 n-1)} X_{N}^{*} D_{N}^{(2 n)} X_{N}\right)_{h_{N}(t), h_{N}(t)} \\
=\sum_{\pi \in \mathcal{P}(n)} g_{1}(t) \Lambda_{\pi}\left(g_{3}, g_{5}, \ldots, g_{2 n-1}\right)(t) \tau\left(\Gamma_{\pi}\left(g_{2}, g_{4}, \ldots, g_{2 n}\right)\right),
\end{aligned}
$$

and

$$
\begin{aligned}
\lim _{N \rightarrow \infty} \mathbb{E}\left(D_{N}^{(1)} X_{N} D_{N}^{(2)} X_{N}^{*} D_{N}^{(3)} X_{N} D_{N}^{(4)} X_{N}^{*} \cdots D_{N}^{(2 n-1)} X_{N} D_{N}^{(2 n)} X_{N}^{*}\right)_{h_{N}(t), h_{N}(t)} \\
=\sum_{\pi \in \mathcal{P}(n)} \Gamma_{\pi}\left(g_{1}, g_{3}, \ldots, g_{2 n-1}\right)(t) \tau\left(\Lambda_{\pi}\left(g_{2}, g_{4}, \ldots, g_{2 n-2}\right) g_{2 n}\right)
\end{aligned}
$$

Furthermore, in both cases the convergence is uniform for $t \in[0,1]$, and the rate of convergence can be controlled in terms of only $\max _{i}\left\|g_{i}\right\|$ and a common modulus of continuity for $\left\{g_{1}, \ldots, g_{2 n}\right\}$. 
Proof. We have

$$
\begin{aligned}
& \mathbb{E}\left(D_{N}^{(1)} X_{N}^{*} D_{N}^{(2)} X_{N} D_{N}^{(3)} X_{N}^{*} D_{N}^{(4)} X_{N} \cdots D_{N}^{(2 n-1)} X_{N}^{*} D_{N}^{(2 n)} X_{N}\right)_{h_{N}(t), h_{N}(t)} \\
&=N_{\left\{(i(1), \ldots, i(2 n)) \in\{1, \ldots, N\}^{2 n}: i(1)=h_{N}(t)\right\}}\left(\prod_{j=1}^{2 n} g_{j}\left(\frac{i(j)}{N}\right)\right) \\
& \cdot \mathbb{E}\left(\zeta_{i(2)}^{-i(1)+i(3)} \zeta_{i(4)}^{-i(3)+i(5)} \cdots \zeta_{i(2 n-2)}^{-i(2 n-3)+i(2 n-1)} \zeta_{i(2 n)}^{-i(2 n-1)+i(1)}\right) .
\end{aligned}
$$

Let us rearrange the sum by summing first over all partitions $\pi \in \mathcal{P}(n)$ and then over all $i_{e}=(i(2), i(4), \ldots, i(2 n)) \in\{1, \ldots, N\}^{n}$ such that ker $i_{e}=\pi$, and then over all $i_{o}=(i(1), i(3), \ldots, i(2 n-1)) \in\{1, \ldots, N\}^{n}$ such that $i(1)=h_{N}(t)$, where ker $i_{e}=\pi$ means that $i\left(2 j_{1}\right)=i\left(2 j_{2}\right)$ if and only if $j_{1}$ and $j_{2}$ are in the same block of $\pi$. Keeping in mind that the $\zeta_{j}$ are independent and $\mathbb{E}\left(\zeta_{j}^{k}\right)=0$ if $k \neq 0$, we find that the expectation in (4.6) equals

$$
N^{-n} \sum_{\pi \in \mathcal{P}(n)}\left(\sum_{\ell} \prod_{S \in \pi}\left(\prod_{j \in S} g_{2 j}\left(\frac{\ell_{S}}{N}\right)\right)\right) \sum_{i_{o} \in \Psi_{1}\left(\pi, N, h_{N}(t)\right)} \prod_{j=1}^{n} g_{2 j-1}\left(\frac{i(2 j-1)}{N}\right)
$$

where the summation $\sum_{\ell}$ is over all $\ell=\left(\ell_{S}\right)_{S \in \pi} \in\{1, \ldots, N\}^{|\pi|}$ such that $\ell_{S_{1}} \neq \ell_{S_{2}}$ if $S_{1} \neq S_{2}$, and $\Psi_{1}\left(\pi, N, h_{N}(t)\right)$ is the set of all $i_{o}=(i(1), i(3), \ldots, i(2 n-1)) \in$ $\{1, \ldots, N\}^{n}$ such that $i(1)=h_{N}(t)$ and for all $S \in \pi$,

$$
\sum_{j \in S}-i(2 j-1)+i(2 j+1)=0
$$

with the convention $i(2 n+1)=i(1)$. It is straightforward from the theory of Riemann integration to see

$$
\lim _{N \rightarrow \infty} N^{-|\pi|}\left(\sum_{\ell} \prod_{S \in \pi}\left(\prod_{j \in S} g_{2 j}\left(\frac{\ell_{S}}{N}\right)\right)\right)=\prod_{s \in \pi} \tau\left(\prod_{j \in S} g_{2 j}\right)
$$


and that the rate of convergence depends only on $\max _{j}\left\|g_{2 j}\right\|$ and on a common modulus of continuity of $\left\{g_{2}, g_{4}, \ldots, g_{2 n}\right\}$

Now, to analyze the last summation in (4.7), we have a bijection $\Psi_{1}\left(\pi, N, h_{N}(t)\right) \rightarrow \Phi_{1}\left(\pi, N, h_{N}(t)\right)$ given by

$$
\begin{aligned}
& (i(1), i(3), \ldots, i(2 n-1)) \\
& \mapsto(-i(1)+i(3),-i(3)+i(5), \ldots,-i(2 n-3)+i(2 n-1),-i(2 n-1)+i(1)),
\end{aligned}
$$

where $\Phi_{1}\left(\pi, N, h_{N}(t)\right)$ is the set of all $f=(f(1), \ldots, f(n)) \in \mathbf{Z}^{n}$ such that

$$
\begin{gathered}
\forall S \in \pi, \quad \sum_{j \in S} f(j)=0, \\
\forall p \in\{1, \ldots, n-1\}, \quad 1 \leq h_{N}(t)+\sum_{j=1}^{p} f(j) \leq N .
\end{gathered}
$$

Let $\Phi_{1}^{\prime}\left(\pi, N, h_{N}(t)\right)$ be the set of all maps $f: J \rightarrow \mathbf{Z}$, where $J$ is as in (4.1), such that

$$
\forall p \in\{1, \ldots, n-1\}, \quad 1 \leq h_{N}(t)+\sum_{j \in I_{\pi}(p)} f(j) \leq N .
$$

Then the map $\Phi_{1}\left(\pi, N, h_{N}(t)\right) \rightarrow \Phi_{1}^{\prime}\left(\pi, N, h_{N}(t)\right)$, given by restriction, i.e., $f \mapsto f \uparrow_{J}$, is a bijection. Moreover, if $f \in \Phi_{1}^{\prime}\left(\pi, N, h_{N}(t)\right)$ and if $(i(1), i(3), \ldots, i(2 n-1))$ is the corresponding element of $\Psi_{1}\left(\pi, N, h_{N}(t)\right)$, then for all $p \in\{1,2, \ldots, n-1\}$ we have

$$
i(2 p+1)=h_{N}(t)+\sum_{j \in I_{\pi}(p)} f(j)
$$


so also

$$
\begin{aligned}
\sum_{i_{o} \in \Psi_{1}\left(\pi, N, h_{N}(t)\right)} \prod_{j=1}^{n} g_{2 j-1}\left(\frac{i(2 j-1)}{N}\right) & \\
& =\sum_{f \in \Phi_{1}^{\prime}\left(\pi, N, h_{N}(t)\right)} g_{1}\left(\frac{h_{N}(t)}{N}\right) \prod_{p=1}^{n-1} g_{2 p+1}\left(\frac{h_{N}(t)}{N}+\sum_{j \in I_{\pi}(p)} \frac{f(j)}{N}\right) .
\end{aligned}
$$

Note that $\left\{f / N: f \in \Phi_{1}^{\prime}\left(\pi, N, h_{N}(t)\right)\right\}$ is an evenly spaced grid of points that fills out the set $E(\pi, t)$ as $N \rightarrow \infty$ and that the above sums, when divided by $N^{n-|\pi|}$, are Riemann sums for multivariate Riemann integrals. Using standard estimates, we find

$$
\lim _{N \rightarrow \infty} N^{-n+|\pi|} \sum_{i_{o} \in \Psi_{1}\left(\pi, N, h_{N}(t)\right)} \prod_{j=1}^{n} g_{2 j-1}\left(\frac{i(2 j-1)}{N}\right)=g_{1}(t) \Lambda_{\pi}\left(g_{3}, g_{5}, \ldots, g_{2 n-1}\right)(t)
$$

and that the rate of convergence depends only on $\max _{1 \leq j \leq n}\left\|g_{2 j-1}\right\|$ and on a common modulus of continuity of $\left\{g_{1}, g_{3}, \ldots, g_{2 n-1}\right\}$. This proves (4.4), with the desired statement on the rate of convergence.

We prove (4.5) similarly. We have

$$
\begin{aligned}
& \mathbb{E}\left(D_{N}^{(1)} X_{N} D_{N}^{(2)} X_{N}^{*} D_{N}^{(3)} X_{N} D_{N}^{(4)} X_{N}^{*} \cdots\right.\left.D_{N}^{(2 n-1)} X_{N} D_{N}^{(2 n)} X_{N}^{*}\right)_{h_{N}(t), h_{N}(t)} \\
&=N^{-n} \sum_{\left\{(i(1), \ldots, i(2 n)) \in\{1, \ldots, N\}^{2 n}: i(1)=h_{N}(t)\right\}}\left(\prod_{j=1}^{2 n} g_{j}\left(\frac{i(j)}{N}\right)\right) \\
& \cdot \mathbb{E}\left(\zeta_{i(1)}^{-i(2 n)+i(2)} \zeta_{i(3)}^{-i(2)+i(4)} \cdots \zeta_{i(2 n-1)}^{-i(2 n-2)+i(2 n)}\right) .
\end{aligned}
$$

The right-hand-side can be rewritten

$$
N^{-n} \sum_{\pi \in \mathcal{P}(n)}\left(\sum_{\ell} \prod_{S \in \pi}\left(\prod_{j \in S} g_{2 j-1}\left(\frac{\ell_{S}}{N}\right)\right)\right) \sum_{i_{e} \in \Psi_{2}(\pi, N)} \prod_{j=1}^{n} g_{2 j}\left(\frac{i(2 j)}{N}\right)
$$


where the summation $\sum_{\ell}$ is over all $\ell=\left(\ell_{S}\right)_{S \in \pi} \in\{1, \ldots, N\}^{|\pi|}$ such that $\ell_{S_{\pi}(1)}=$ $h_{N}(t)$ and $\ell_{S_{1}} \neq \ell_{S_{2}}$ if $S_{1} \neq S_{2}$, while

$$
\begin{aligned}
& \Psi_{2}(\pi, N) \\
= & \left\{(i(2), i(4), \ldots, i(2 n)) \in\{1, \ldots, N\}^{n}: \forall S \in \pi, \sum_{j \in S} i(2 j)-i(2 j-2)=0\right\},
\end{aligned}
$$

with the convention $i(0)=i(2 n)$. We see

$$
\lim _{N \rightarrow \infty} N^{-|\pi|+1} \sum_{\ell} \prod_{S \in \pi}\left(\prod_{j \in S} g_{2 j-1}\left(\frac{\ell_{S}}{N}\right)\right)=\Gamma_{\pi}\left(g_{1}, g_{3}, \ldots, g_{2 n-1}\right)(t) .
$$

We use the bijection $\Psi_{2}(\pi, N) \rightarrow \Phi_{2}(\pi, N)$ given by

$$
\begin{aligned}
& (i(2), i(4), \ldots, i(2 n)) \\
& \quad \mapsto(i(2 n),-i(2 n)+i(2),-i(2)+i(4),-i(4)+i(6), \ldots,-i(2 n-2)+i(2 n)),
\end{aligned}
$$

where $\Phi_{2}(\pi, N)$ is the set of all $(f(0), f(1), f(2), \ldots, f(n)) \in \mathbf{Z}^{n+1}$ such that

$$
\begin{gathered}
\forall S \in \pi \quad \sum_{j \in S} f(j)=0 \\
\forall p \in\{1, \ldots, n\} \quad 1 \leq f(0)+\sum_{j=1}^{p} f(j) \leq N
\end{gathered}
$$

We let $\Phi_{2}^{\prime}(\pi, N)$ be the set of all maps $f: J \cup\{0\} \rightarrow \mathbf{Z}$ such that

$$
\forall p \in\{1, \ldots, n\} \quad 1 \leq f(0)+\sum_{j \in I_{\pi}(p)} f(j) \leq N
$$

and we note that the map $\Phi_{2}(\pi, N) \rightarrow \Phi_{2}^{\prime}(\pi, N)$ given by restriction, namely, $f \mapsto$ $f \uparrow_{J \cup\{0\}}$, is a bijection. Moreover, if $f \in \Phi_{2}^{\prime}(\pi, N)$ and if $(i(1), i(3), \ldots, i(2 n-1))$ is 
the corresponding element of $\Psi_{2}(\pi, N)$, then for all $p \in\{1,2, \ldots, n\}$ we have

$$
i(2 p)=f(0)+\sum_{j \in I_{\pi}(p)} f(j)
$$

Thus, in particular, $i(2 n)=f(0)$. Hence, we also have

$$
\sum_{i_{e} \in \Psi_{2}(\pi, N)} \prod_{j=1}^{n} g_{2 j}\left(\frac{i(2 j)}{N}\right)=\sum_{f \in \Phi_{2}^{\prime}(\pi, N)} \prod_{p=1}^{n} g_{2 p}\left(\frac{f(0)}{N}+\sum_{j \in I_{\pi}(p)} \frac{f(j)}{N}\right) .
$$

We have that $\left\{f / N: f \in \Phi_{2}^{\prime}(\pi, N)\right\}$ is an evenly spaced grid of points that fills out the set $\cup_{0 \leq s \leq 1} E(\pi, s)$ as $N \rightarrow \infty$ and that the above sums, when divided by $N^{n-|\pi|+1}$, are Riemann sums. Using standard estimates, we find

$$
\lim _{N \rightarrow \infty} N^{-n+|\pi|-1} \sum_{i_{e} \in \Psi_{2}(\pi, N)} \prod_{j=1}^{n} g_{2 j}\left(\frac{i(2 j)}{N}\right)=\int_{0}^{1} \Lambda_{\pi}\left(g_{2}, g_{4}, \ldots, g_{2 n-2}\right)(s) g_{2 n}(s) d s
$$

and that the rate of convergence depends only on $\max _{1 \leq j \leq n}\left\|g_{2 j}\right\|$ and on a common modulus of continuity of $\left\{g_{2}, g_{4}, \ldots, g_{2 n}\right\}$. This proves (4.5), with the desired statement on the rate of convergence.

\section{$4.3 C[0,1]$-valued R-diagonality}

In this subsection, we prove our main theorem (Theorem 4.3.1) about asymptotic *-moments of random Vandermonde matrices. It will follow from Proposition 4.1 above, about alternating moments, and the next proposition.

Proposition 4.2. Let $n \geq 1$. Let $\epsilon_{1}, \ldots, \epsilon_{n} \in\{1, *\}$. Let $\sigma$ be the interval partition of $\{1, \ldots, n\}$ defined by

$$
k \stackrel{\sigma}{\sim} k+1 \Longleftrightarrow \epsilon_{k} \neq \epsilon_{k+1}
$$

for $k \in\{1, \ldots, n-1\}$. Let $d_{1}, \ldots, d_{n}$ be deterministic diagonal $N \times N$ matrices of 
norm at most 1 . Then

$$
\left|\mathbb{E} \circ \operatorname{tr} \prod_{I \in \sigma}\left(\prod_{k \in I} d_{k} X_{N}^{\epsilon_{k}}-\mathbb{E} \circ \operatorname{diag}\left(\prod_{k \in I} d_{k} X_{N}^{\epsilon_{k}}\right)\right)\right| \leq \frac{C}{\sqrt{N}}
$$

where $C$ depends only on $n$.

We begin with some preliminaries. The following lemma can be proven using Gaussian elimination, for instance.

Lemma 4.3. Let $p \geq 1$. Let $V$ be a subspace of $\mathbb{R}^{p}$. Let $t \in \mathbb{R}^{p}$. Then

$$
\mid\left\{j \in\{1, \ldots, N\}^{\{1, \ldots, p\}} \cap(t+V) \mid \leq N^{\operatorname{dim} V} .\right.
$$

Lemma 4.3 can be reformulated follows.

Lemma 4.4. Let $p, r \geq 1$. Let $w_{1}, \ldots, w_{r} \in \mathbb{R}^{p}$. Let $m_{1}, \ldots, m_{r} \in \mathbb{R}$. Then

$$
\left|\left\{j \in\{1, \ldots, N\}^{\{1, \ldots, p\}}: j \cdot w_{s}=m_{s} \forall 1 \leq s \leq r\right\}\right| \leq N^{p-\operatorname{dim} \operatorname{span}}\left\{w_{1}, \ldots, w_{r}\right\} .
$$

Lemma 4.5. Let $\zeta_{1}, \ldots, \zeta_{N}$ be independent random variables uniformly distributed on the unit circle. Let $h$ be a product of the random variables $\zeta_{1}, \ldots, \zeta_{N}$ and their inverses, possibly with repetitions. Then

$$
\mathbb{E} h=\left\{\begin{array}{cc}
1, & h=1 \\
0, & h \neq 1
\end{array}\right.
$$

Proof. Obviously if $h=1$ then $\mathbb{E} h=1$. If $h \neq 1$ then we write $h=\prod_{i=1}^{N} \zeta_{i}^{j(i)}$ where $j\left(i_{0}\right) \neq 0$ for some $1 \leq i_{0} \leq N$. Thus, by independence of $\zeta_{1}, \ldots, \zeta_{N}$, we have $\mathbb{E} h=\left(\mathbb{E} \prod_{i \neq i_{0}} \zeta_{i}^{j(i)}\right)\left(\mathbb{E} \zeta_{i_{0}}^{j\left(i_{0}\right)}\right)=0$. 
Lemma 4.6. Let $\zeta_{1}, \ldots, \zeta_{N}$ be independent random variables uniformly distributed on the unit circle. Let $h_{1}, \ldots, h_{r}$ be products of the random variables $\zeta_{1}, \ldots, \zeta_{N}$ and their inverses, possibly with repetition. Then

$$
\left|\mathbb{E}\left(h_{1}-\mathbb{E} h_{1}\right) \cdots\left(h_{r}-\mathbb{E} h_{r}\right)\right| \leq \mathbb{E} h_{1} \cdots h_{r} .
$$

Proof. If $h_{i}=1$ for some $1 \leq i \leq r$ then

$$
\left|\mathbb{E}\left(h_{1}-\mathbb{E} h_{1}\right) \cdots\left(h_{r}-\mathbb{E} h_{r}\right)\right|=0 .
$$

If $h_{i} \neq 1$ for all $1 \leq i \leq r$ then by Lemma $4.5, \mathbb{E} h_{i}=0$ for all $1 \leq i \leq r$ so

$$
\left|\mathbb{E}\left(h_{1}-\mathbb{E} h_{1}\right) \cdots\left(h_{r}-\mathbb{E} h_{r}\right)\right|=\left|\mathbb{E} h_{1} \cdots h_{r}\right|=\mathbb{E} h_{1} \cdots h_{r} .
$$

Lemma 4.7. Let $\zeta_{1}, \ldots, \zeta_{N}$ be independent random variables uniformly distributed on the unit circle. Let $h$ be a product of the random variables $\zeta_{1}, \ldots, \zeta_{N}$ and their inverses, possibly with repetition. Let $r \geq 1$. Let $i(1), \ldots, i(r) \in\{1, \ldots, N\}$ be distinct. Then there exists $m_{1}, \ldots, m_{r} \in \mathbb{Z}$ such that if $n_{1}, \ldots, n_{r} \in \mathbb{Z}$ satisfies

$$
\mathbb{E} h \zeta_{i(1)}^{n_{1}} \cdots \zeta_{i(r)}^{n_{r}} \neq 0
$$

then $n_{s}=m_{s}$ for all $1 \leq s \leq r$.

Proof. We write $h$ as $\prod_{i=1}^{N} \zeta_{i}^{j(i)}$. Then the result follows from Lemma 4.5, by taking $m_{s}=-j(i(s))$ for $1 \leq s \leq r$.

Combining Lemma 4.4 and Lemma 4.7, we obtain 
Lemma 4.8. Let $p, r \geq 1$. Let $w_{1}, \ldots, w_{r} \in \mathbb{R}^{p}$. Let $\zeta_{1}, \ldots, \zeta_{N}$ be independent random variables uniformly distributed on the unit circle. Let $h$ be a product of the random variables $\zeta_{1}, \ldots, \zeta_{N}$ and their inverses, possibly with repetition. Let $i(1), \ldots, i(r) \in\{1, \ldots, N\}$ be distinct. Then

$$
\left|\left\{j \in\{1, \ldots, N\}^{\{1, \ldots, p\}}: \mathbb{E} h \zeta_{i(1)}^{j \cdot w_{1}} \cdots \zeta_{i(r)}^{j \cdot w_{r}} \neq 0\right\}\right| \leq N^{p-\operatorname{dim} \operatorname{span}\left\{w_{1}, \ldots, w_{r}\right\}} .
$$

Equivalently, by Lemma 4.5,

$$
\sum_{j:\{1, \ldots, p\} \rightarrow\{1, \ldots, N\}}\left|\mathbb{E} h \zeta_{i(1)}^{j \cdot w_{1}} \cdots \zeta_{i(r)}^{j \cdot w_{r}}\right| \leq N^{p-\operatorname{dim} \operatorname{span}\left\{w_{1}, \ldots, w_{r}\right\}}
$$

Lemma 4.9. Let $K$ be a finite set. Let $\pi_{1}, \pi_{2}$ be partitions of $K$. Let $\left(v_{k}\right)_{k \in K}$ be a finite collection of vectors in a vector space $V$ such that whenever $\left(a_{k}\right)_{k \in K}$ are scalars satisfying

$$
\sum_{k \in K} a_{k} v_{k}=0
$$

we have $a_{k}=a_{l}$ for all $k \stackrel{\pi_{2}}{\sim} l$. Then

$$
\operatorname{dimspan}\left\{\sum_{k \in S} v_{k}: S \in \pi_{1}\right\} \geq\left|\pi_{1}\right|-\left|\pi_{1} \vee \pi_{2}\right|
$$

Proof. Let $\left(a_{S}\right)_{S \in \pi_{1}}$ be scalars such that

$$
\sum_{S \in \pi_{1}} a_{S}\left(\sum_{k \in S} v_{k}\right)=0
$$

For $k \in K$, let $S(k)$ be the block in $\pi_{1}$ containing $k$. Then

$$
0=\sum_{S \in \pi_{1}} a_{S}\left(\sum_{k \in S} v_{k}\right)=\sum_{S \in \pi_{1}} \sum_{k \in S} a_{S} v_{k}=\sum_{S \in \pi_{1}} \sum_{k \in S} a_{S(k)} v_{k}=\sum_{k \in K} a_{S(k)} v_{k} .
$$


So by assumption, $a_{S(k)}=a_{S(l)}$ for all $k \stackrel{\pi_{2}}{\sim} l$. Hence, $a_{S(k)}=a_{S(l)}$ for all $k \stackrel{\pi_{1} \vee \pi_{2}}{\sim} l$. Therefore,

$$
\operatorname{dim}\left\{\left(a_{S}\right)_{S \in \pi_{1}}: \sum_{S \in \pi_{1}} a_{S}\left(\sum_{k \in S} v_{k}\right)=0\right\} \leq\left|\pi_{1} \vee \pi_{2}\right| .
$$

Thus, the result follows.

Lemma 4.10. Let $K \subset L$ be finite sets. Let $\pi$ be a partition of $L$. Let $\lambda$ be a partition of $K$. Then $\lambda \cup\{\{l\}: l \in L \backslash K\}$ is a partition of $L$ and

$$
\mid\left(\pi \lceil _ { K } ) \vee \lambda | + | \pi | - | \pi \left\lceil_{K}|=| \pi \vee(\lambda \cup\{\{l\}: l \in L \backslash K\}) \mid .\right.\right.
$$

Proof. Let $K^{\prime}$ be the union of all blocks in $\pi$ that contain an element in $K$. Then

$$
\begin{gathered}
\left|(\pi \vee(\lambda \cup\{\{l\}: l \in L \backslash K\})) \uparrow_{K^{\prime}}\right|=\left|(\pi \vee(\lambda \cup\{\{l\}: l \in L \backslash K\})) \uparrow_{K}\right|=\left|\left(\pi \uparrow_{K}\right) \vee \lambda\right|, \\
(\pi \vee(\lambda \cup\{\{l\}: l \in L \backslash K\})) \uparrow_{L \backslash K^{\prime}}=\pi \uparrow_{L \backslash K^{\prime}},
\end{gathered}
$$

and

$$
\mid \pi\left\lceil_ { L \backslash K ^ { \prime } } | = | \pi | - | \pi \left\lceil_{K^{\prime}} \mid .\right.\right.
$$

Since $K^{\prime}$ splits the partition $\pi \vee(\lambda \cup\{\{l\}: l \in L \backslash K\})$, we have

$$
\begin{aligned}
\mid \pi & \vee(\lambda \cup\{\{l\}: l \in L \backslash K\}) \mid \\
& =\left|(\pi \vee(\lambda \cup\{\{l\}: l \in L \backslash K\})) \uparrow_{K^{\prime}}\right|+\left|(\pi \vee(\lambda \cup\{\{l\}: l \in L \backslash K\})) \uparrow_{L \backslash K^{\prime}}\right| \\
& =\left|\left(\pi \uparrow_{K}\right) \vee \lambda\right|+|\pi|_{L \backslash K^{\prime}}|=|\left(\pi \uparrow_{K}\right) \vee \lambda|+| \pi|-| \pi\left\lceil_{K^{\prime}} \mid .\right.
\end{aligned}
$$

Lemma 4.11. Let $\pi_{1}, \pi_{2}$ be a partitions of L. If $\left|\pi_{1} \vee \pi_{2}\right|>\frac{1}{2}\left|\pi_{2}\right|$ then there exists a block $S \in \pi_{2}$ that splits $\pi_{1}$. 
The proof of Lemma 4.11 is analogous to the proof of the fact that a partition of $n$ points with more than $\frac{n}{2}$ blocks must contain a singleton block.

Lemma 4.11 can be reformulated as

Lemma 4.12. Let $L$ be a finite set. Let $i: L \rightarrow\{1, \ldots, N\}$. Let $\rho$ be a partition of L. If $|(\operatorname{ker} i) \vee \rho|>\frac{1}{2}|\rho|$ then there exists a block $S \in \rho$ such that $\{i(l): l \in S\}$ and $\{i(l): l \in L \backslash S\}$ are disjoint.

Lemma 4.13. Let $U$ be a finite set in $\mathbb{Z}$. For each $k \in(U-1) \cup(U+1)$, define the vector $v_{k} \in \mathbb{R}^{U}$ as

$$
v_{k}=\left\{\begin{array}{cl}
e_{k+1}, & k \in(U-1) \backslash(U+1) \\
e_{k+1}-e_{k-1}, & k \in(U-1) \cap(U+1) \\
-e_{k-1}, & k \in(U+1) \backslash(U-1)
\end{array}\right.
$$

If

$$
\sum_{k \in(U-1) \cup(U+1)} a_{k} v_{k}=0,
$$

then $a_{l-1}=a_{l+1}$ for all $l \in U$.

Proof. Let $l \in U$. Then

$$
\sum_{k \in(U-1) \cup(U+1)} a_{k}\left\langle v_{k}, e_{l}\right\rangle=0 .
$$

Since $v_{k} \in \operatorname{span}\left\{e_{k-1}, e_{k+1}\right\}$ for all $k \in(U-1) \cup(U+1)$, if $l \neq k+1$ and $l \neq k-1$ then $\left\langle v_{k}, e_{l}\right\rangle=0$. Thus, the only values of $k$ for which $\left\langle v_{k}, e_{l}\right\rangle$ can possibly be nonvanishing are $l-1$ and $l+1$. Hence,

$$
a_{l+1}\left\langle v_{l+1}, e_{l}\right\rangle+a_{l-1}\left\langle v_{l-1}, e_{l}\right\rangle=0 .
$$


Since $l \in U$, we have:

- $l+1 \in U+1$ and $l-1 \in U-1$,

- if $l+1 \in U-1$ then $v_{l+1}=e_{l+2}-e_{l}$ and $\left\langle v_{l+1}, e_{l}\right\rangle=-1$,

- if $l+1 \notin U-1$ then $v_{l+1}=-e_{l}$ and $\left\langle v_{l+1}, e_{l}\right\rangle=-1$,

- if $l-1 \in U+1$ then $v_{l-1}=e_{l}-e_{l+1}$ and $\left\langle v_{l-1}, e_{l}\right\rangle=1$,

- if $l-1 \notin U+1$ then $v_{l-1}=e_{l}$ and $\left\langle v_{l-1}, e_{l}\right\rangle=1$.

In all of the above cases, $\left\langle v_{l+1}, e_{l}\right\rangle=-1$ and $\left\langle v_{l-1}, e_{l}\right\rangle=1$. Therefore, $-a_{l+1}+a_{l-1}=$ 0. So $a_{l-1}=a_{l+1}$.

Lemma 4.14. Let $U$ be a finite set in $\mathbb{Z}$. Let $\sim$ be the equivalence relation on $(U-1) \cup(U+1)$ generated by $l-1 \sim l+1(l \in U)$. Then this equivalence relation has at most $|(U+1) \backslash(U-1)|$ equivalence classes.

Proof. It suffices to show that every element $k$ of $(U-1) \cup(U+1)$ is related to an element in $(U+1) \backslash(U-1)$. If $k \in U+1$ then $k \sim k-2 \in U-1$. So replacing $k$ by $k-2$, if necessary, we may assume that $k \in U-1$. Let $p$ be smallest natural number for which $k+2 p \notin U-1$. By minimality, $k+2 q \in U-1$ for all $0 \leq q \leq p-1$. So $k+2 q+1 \in U$ so by assumption, $k+2 q \sim k+2 q+2$ for all $0 \leq q \leq p-1$. Therefore,

$$
k \sim k+2 \sim k+4 \sim \ldots \sim k+2 p
$$

Since $k+2(p-1) \in U-1, k+2 p \in U+1$. Hence, $k+2 p \in(U+1) \backslash(U-1)$.

Lemma 4.15. Let $n \geq 1$. Let $\epsilon_{1}, \ldots, \epsilon_{n} \in\{1, *\}$. Let $\sigma$ be the interval partition of $\{1, \ldots, n\}$ defined by

$$
k \stackrel{\sigma}{\sim} k+1 \Longleftrightarrow \epsilon_{k} \neq \epsilon_{k+1}
$$


For each $I \in \sigma$, let

$$
L(I)=\left\{k \in I: \epsilon_{k}=1\right\} \cup\left\{k+1: k \in I \text { and } \epsilon_{k}=*\right\} .
$$

Then $L\left(I_{1}\right) \cap L\left(I_{2}\right)=\emptyset$ for all distinct $I_{1}, I_{2} \in \sigma$.

Proof. Let $k \in I_{2}$ with $\epsilon_{k}=*$. If $k+1 \notin I_{2}$, then since $I_{2} \in \sigma$, by the definition of $\sigma, \epsilon_{k+1}=*$. On the other hand, if $k+1 \in I_{2}$, then since $I_{1}$ and $I_{2}$ are disjoint blocks and are, therefore, disjoint, $k+1 \notin I_{1}$. In both cases, we have that either $k+1 \notin I_{1}$ or $\epsilon_{k+1} \neq 1$. Hence,

$$
\left\{k+1: k \in I_{2} \text { and } \epsilon_{k}=*\right\} \cap\left\{k \in I_{1}: \epsilon_{k}=1\right\}=\emptyset .
$$

Interchanging the roles of $I_{1}$ and $I_{2}$, we have

$$
\left\{k+1: k \in I_{1} \text { and } \epsilon_{k}=*\right\} \cap\left\{k \in I_{2}: \epsilon_{k}=1\right\}=\emptyset .
$$

Since $I_{1}$ and $I_{2}$ are disjoint,

$$
\left\{k \in I_{1}: \epsilon_{k}=1\right\} \cap\left\{k \in I_{2}: \epsilon_{k}=1\right\}=\emptyset
$$

and

$$
\left\{k+1: k \in I_{1} \text { and } \epsilon_{k}=*\right\} \cap\left\{k+1: k \in I_{2} \text { and } \epsilon_{k}=*\right\}=\emptyset \text {. }
$$

Therefore, $L\left(I_{1}\right) \cap L\left(I_{2}\right)=\emptyset$.

Lemma 4.16. Let $\epsilon_{1}, \ldots, \epsilon_{n} \in\{1, *\}$. Let $\sigma$ be the interval partition defined in 
Lemma 4.15 and let $L(I)$ for $I \in \sigma$ be as defined there. Let

$$
U=\left\{2 \leq k \leq n: \epsilon_{k-1}=1 \text { and } \epsilon_{k}=*\right\} .
$$

Let $\sim$ be the equivalence relation on $(U-1) \cup(U+1)$ generated by $l-1 \sim l+1$ for $l \in U$. Then

(i) every equivalence class of $\sim$ is of the form $L(I)$ for some $I \in \sigma$

(ii) for every $l \in\{2, \ldots, n\} \backslash(U \cup(U-1) \cup(U+1))$, there exists $I \in \sigma$ such that $\{l\}=L(I)$.

Proof. We will first prove (i). Let

$$
\sigma_{0}=\{I \in \sigma: I \cap(U-1) \neq \emptyset\}
$$

We want to show that for every $I \in \sigma_{0}, L(I)$ is an equivalence class of $\sim$. After proving this, we show that $\cup_{I \in \sigma_{0}} L(I)=(U-1) \cup(U+1)$. This immediately gives the conclusion of (i), because $\{L(I)\}_{I \in \sigma_{0}}$ is the partition of $(U-1) \cup(U+1)$ that corresponds to the equivalence relation $\sim$.

1. We first show that $L(I) \subset(U-1) \cup(U+1)$ for every $I \in \sigma_{0}$. Since $I \in \sigma_{0}$, there exists $l \in I$ such that $\epsilon_{l}=1$ and $\epsilon_{l+1}=*$. Since $I \in \sigma$, by the definition of $\sigma$, we have $l+1 \in I$. (In particular, $l$ and $l+1$ are in $I$.)

A. Suppose $k \in I$ and $\epsilon_{k}=1$. We will show $k \in(U-1) \cup(U+1)$. Since $I$ is an interval of length at least 2 , if $k \in I$ then $k+1 \in I$ or $k-1 \in I$. If $k+1 \in I$, then by the definition of $\sigma, \epsilon_{k+1}=*$ so $k+1 \in U$ and $k \in U-1$. Suppose $k+1 \notin I$. Then either $k=n$ or $\epsilon_{k+1}=1$ and in either case, $k+1 \notin U$. Then $k-1 \in I$ and $\epsilon_{k-1}=*$. If $k-2 \in I$, then $\epsilon_{k-2}=1$ and $k-1 \in U$ and 
$k \in U+1$. Otherwise, if $k-2 \notin I$, then either $k=2$ or $\epsilon_{k-2}=*$ and we have $I=\{k-1, k\}$. But $k \notin U$, so $I \cap(U-1)=\emptyset$, contrary to the hypothesis $I \in \sigma_{0}$. Thus, we have shown $\left\{k \in I: \epsilon_{k}=1\right\} \subset(U-1) \cup(U+1)$.

B. Suppose $k \in I$ and $\epsilon_{k}=*$. We will show $k+1 \in(U-1) \cup(U+1)$. Since $I \in \sigma$, by the definition of $\sigma$, we must have $\epsilon_{k-1}=1$ unless $k$ is the smallest element of $I$.

I. If $\epsilon_{k-1}=1$ then $k \in U$ so $k+1 \in U+1$.

II. If $k$ is the smallest element of $I$, then $k \leq l$. Since $\epsilon_{k}=*$ and $\epsilon_{l}=1$, $k \neq l$. So $k+1 \leq l$ so $k+2 \leq l+1 \in I$. Since $I$ is an interval, it follows that $k, k+1, k+2 \in I$. So $\epsilon_{k}=*, \epsilon_{k+1}=1$ and $\epsilon_{k+2}=*$. So $k+2 \in U$ so $k+1 \in U-1$.

Thus, we have shown $\left\{k+1: k \in I\right.$ and $\left.\epsilon_{k}=*\right\} \subset(U-1) \cup(U+1)$.

It follows that $L(I) \subset(U-1) \cup(U+1)$.

2. To show that $L(I)$ is an equivalence class of $\sim$, we will prove that $L(I)$ is preserved by the equivalence relation $\sim$ and that all elements of $L(I)$ are related.

A. Suppose $k_{0} \in U$ and $k_{0}-1 \in L(I)$. Since $k_{0} \in U$, we have

$$
\epsilon_{k_{0}-1}=1, \quad \epsilon_{k_{0}}=*
$$

Since $k_{0}-1 \in L(I)$, either $k_{0}-1 \in\left\{k \in I: \epsilon_{k}=1\right\}$ or $k_{0}-1 \in\left\{k+1: k \in I\right.$ and $\left.\epsilon_{k}=*\right\}$. In the first case, $k_{0}-1 \in I$ and $\epsilon_{k_{0}}=*$ so $k_{0} \in I$. In the second case, $k_{0}-2 \in I$ and $\epsilon_{k_{0}-2}=*$; by (4.10), we have $k_{0} \in I$. In both cases, $k_{0} \in I$ and $\epsilon_{k_{0}}=*$, so $k_{0}+1 \in L(I)$.

On the other hand, if $k_{0} \in U$ and $k_{0}+1 \in L(I)$ then either 
$k_{0}+1 \in\left\{k \in I: \epsilon_{k}=1\right\}$ or $k_{0}+1 \in\left\{k+1: k \in I\right.$ and $\left.\epsilon_{k}=*\right\}$. In the first case, $k_{0}+1 \in I$ and $\epsilon_{k_{0}+1}=1$. Using (4.10), $k_{0}-1 \in I$. In the second case, $k_{0} \in I$. By (4.10), $k_{0}-1 \in I$. In both cases $k_{0}-1 \in I$ and $\epsilon_{k_{0}-1}=1$, so $k_{0}-1 \in L(I)$.

Therefore, $L(I)$ is preserved by the equivalence relation $\sim$.

B. To prove that all elements of $L(I)$ are related, note that since $I$ is an interval with alternating values of $\epsilon_{k},\left\{k \in I: \epsilon_{k}=1\right\}$ is of the form $\left\{k_{0}, k_{0}+2, \ldots, k_{0}+\right.$ $2 p\}$ for some $p \geq 0$ where $\epsilon_{k_{0}}=1, \epsilon_{k_{0}+1}=*, \epsilon_{k_{0}+2}=1, \ldots, \epsilon_{k_{0}+2 p-1}=*$, $\epsilon_{k_{0}+2 p}=1$. Thus, $k_{0}+1, k_{0}+3, \ldots, k_{0}+2 p-1 \in U$. Thus,

$$
k_{0} \sim k_{0}+2 \sim k_{0}+4 \sim \ldots \sim k_{0}+2 p .
$$

This means that all the elements in $\left\{k \in I: \epsilon_{k}=1\right\}$ are related. Using the same argument, one can show that all the elements in $\left\{k+1: k \in I\right.$ and $\left.\epsilon_{k}=*\right\}$ are related. Just as the beginning of the first part of the proof, since $I \in \sigma_{0}$, there exists $l \in I$ such that $\epsilon_{l}=1$ and $\epsilon_{l+1}=*$ (thus also $l+1 \in I$ ). So $l \in\left\{k \in I: \epsilon_{k}=1\right\}$ and $l+2 \in\left\{k+1: k \in I\right.$ and $\left.\epsilon_{k}=*\right\}$. Since $l+1 \in U, l \sim l+2$. Therefore, all elements in $L(I)=\left\{k \in I: \epsilon_{k}=1\right\} \cup\left\{k+1: k \in I\right.$ and $\left.\epsilon_{k}=*\right\}$ are related.

Therefore, $L(I)$ is an equivalence class of $\sim$ for every $I \in \sigma_{0}$.

3. It remains to show that $\cup_{I \in \sigma_{0}} L(I)=(U-1) \cup(U+1)$. Since $L(I) \subset(U-1) \cup(U+1)$ by the first part of the proof, it suffices to show that $(U-1) \cup(U+1) \subset \cup_{I \in \sigma_{0}} L(I)$.

I. If $k_{0} \in U-1$ then $\epsilon_{k_{0}}=1$. Let $I \in \sigma$ contain $k_{0}$. Then $k_{0} \in L(I)$ and $I \in \sigma_{0}$.

II. If $k_{0} \in U+1$ then $\epsilon_{k_{0}-2}=1$ and $\epsilon_{k_{0}-1}=*$. Let $I \in \sigma$ contain $k_{0}-1$. Then $k_{0} \in L(I)$ and $I \in \sigma_{0}$, since $k_{0}-2 \in I \cap(U-1)$. 
This completes the proof of (i).

We now prove (ii). Let $l \in\{2, \ldots, n\} \backslash(U \cup(U-1) \cup(U+1))$.

1. If $\epsilon_{l}=1$, then since $l \notin U-1$, either $l=n$ or $\epsilon_{l+1}=1$. Since $l \notin U+1$, either $l=2$ or $\epsilon_{l-2}=*$ or $\epsilon_{l-1}=1$.

A. If $l<n$ and $\epsilon_{l-1}=1$, then $\epsilon_{l-1}=\epsilon_{l}=\epsilon_{l+1}=1$, which implies $\{l\} \in \sigma$. Moreover, since $\epsilon_{l}=1, L(\{l\})=\{l\}$.

B. If $l=n$ and $\epsilon_{l-1}=1$, then similarly, $\epsilon_{n-1}=\epsilon_{n}=1$ and we have $\{n\} \in \sigma$ and $L(\{n\})=\{n\}$.

C. If $\epsilon_{l-1}=*$ and $2<l<n$, then $\epsilon_{l-2}=*$ and, since $\epsilon_{l}=\epsilon_{l+1}=1$, we have $\{l-1, l\} \in \sigma$ and $L(\{l-1, l\})=\{l\}$.

D. If $e_{n-1}=*$ and if $2<l=n$ or $2=l<n$, then similarly and $\{l-1, l\} \in \sigma$ and $L(\{l-1, l\})=\{l\}$.

E. If $2=l=n$ and $e_{1}=*$, then $\{1,2\} \in \sigma$ and $L(\{1,2\})=\{2\}$.

2. If $\epsilon_{l}=*$, then since $l \notin U$ we have $\epsilon_{l-1}=*$. Since $l \notin U+1$, either $l=2$ or $\epsilon_{l-2}=*$. In either case, we have $\{l-1\} \in \sigma$ and $L(\{l-1\})=\{l\}$.

This completes the proof.

In the sequel, if $A$ is a $N \times N$ random matrix and $p \geq 1$ then

$$
|A|_{p}:=\left(\mathbb{E} \circ \operatorname{tr}\left(A^{*} A\right)^{\frac{p}{2}}\right)^{\frac{1}{p}}
$$

Thus, if $A$ is deterministic then $|A|_{p}=\left(\operatorname{tr}\left(A^{*} A\right)^{\frac{p}{2}}\right)^{\frac{1}{p}}$ is the normalized Schatten $p$ norm. 
Lemma 4.17. Let $A$ be a $N \times N$ random matrix with integrable entries. Let $p \geq 1$. Then

$$
|\mathbb{E} A|_{p} \leq|A|_{p}
$$

Proof. Since $|\cdot|_{p}$ is a norm on deterministic $N \times N$ matrices,

$$
|\mathbb{E} A|_{p} \leq \mathbb{E}\left(\operatorname{tr}\left(A^{*} A\right)^{\frac{p}{2}}\right)^{\frac{1}{p}} \leq\left(\mathbb{E} \circ \operatorname{tr}\left(A^{*} A\right)^{\frac{p}{2}}\right)^{\frac{1}{p}}=|A|_{p},
$$

where the first inequality follows from Jensen's inequality and the second inequality follows from Hölder's inequality.

Lemma 4.18 ([2], Exercise IV.2.7). Let $A_{1}$ and $A_{2}$ be $N \times N$ (deterministic) matrices. Let $p, q, r$ be positive real numbers such that $\frac{1}{p}+\frac{1}{q}=\frac{1}{r}$. Then

$$
\left|A_{1} A_{2}\right|_{r} \leq\left|A_{1}\right|_{p}\left|A_{2}\right|_{q}
$$

Applying Lemma 4.18 repeatedly, one obtains

Lemma 4.19. Let $A_{1}, \ldots, A_{s}$ be $N \times N$ (deterministic) matrices. Let $p_{1}, \ldots, p_{s}, r \geq$ 1 be such that $\frac{1}{p_{1}}+\ldots+\frac{1}{p_{s}}=\frac{1}{r}$. Then

$$
\left|A_{1} \ldots A_{s}\right|_{r} \leq\left|A_{1}\right|_{p_{1}} \ldots\left|A_{s}\right|_{p_{s}}
$$

Applying the above to random matrices, we get the following:

Lemma 4.20. Let $A_{1}, \ldots, A_{s}$ be $N \times N$ random matrices having finite moments of all orders. Let $p_{1}, \ldots, p_{s}, r \geq 1$ be such that $\frac{1}{p_{1}}+\cdots+\frac{1}{p_{s}}=\frac{1}{r}$. Then

$$
\left|A_{1} \ldots A_{s}\right|_{r} \leq\left|A_{1}\right|_{p_{1}} \ldots\left|A_{s}\right|_{p_{s}}
$$


Proof. By Lemma 4.19,

$$
\operatorname{tr}\left(\left(A_{1} \cdots A_{s}\right)^{*}\left(A_{1} \cdots A_{s}\right)\right)^{\frac{r}{2}} \leq\left(\operatorname{tr}\left(A_{1}^{*} A_{1}\right)^{\frac{p_{1}}{2}}\right)^{\frac{r}{p_{1}}} \cdots\left(\operatorname{tr}\left(A_{s}^{*} A_{s}\right)^{\frac{p_{s}}{2}}\right)^{\frac{r}{p_{s}}}
$$

Taking expectations and using Hölder's inequality, we obtain

$$
\begin{aligned}
\mathbb{E} \circ \operatorname{tr}\left(\left(A_{1} \cdots A_{s}\right)^{*}\left(A_{1} \cdots A_{s}\right)\right)^{\frac{r}{2}} & \leq \mathbb{E}\left(\left(\operatorname{tr}\left(A_{1}^{*} A_{1}\right)^{\frac{p_{1}}{2}}\right)^{\frac{r}{p_{1}}} \cdots\left(\operatorname{tr}\left(A_{s}^{*} A_{s}\right)^{\frac{p_{s}}{2}}\right)^{\frac{r}{p_{s}}}\right) \\
& \leq\left(\mathbb{E} \circ \operatorname{tr}\left(A_{1}^{*} A_{1}\right)^{\frac{p_{1}}{2}}\right)^{\frac{r}{p_{1}}} \cdots\left(\mathbb{E} \circ \operatorname{tr}\left(A_{s}^{*} A_{s}\right)^{\frac{p_{s}}{2}}\right)^{\frac{r}{p_{s}}} \\
& =\left|A_{1}\right|_{p_{1}}^{r} \cdots\left|A_{s}\right|_{p_{s}}^{r} .
\end{aligned}
$$

Thus, (4.11) holds.

Lemma 4.21. Let $A_{1}^{(1)}, \ldots, A_{s}^{(1)}, A_{1}^{(2)}, \ldots, A_{s}^{(2)}$ be $N \times N$ random matrices having finite moments of all orders. Let $M=\max \left\{\left|A_{l}^{(1)}\right|_{2(s-1)},\left|A_{l}^{(2)}\right|_{2(s-1)}: 1 \leq l \leq s\right\}$ if $s \geq 2$ and let $M=1$ if $s=1$. Then

$$
\left|\mathbb{E} \circ \operatorname{tr}\left(\prod_{l=1}^{s}\left(A_{l}^{(1)}+A_{l}^{(2)}\right)\right)-\mathbb{E} \circ \operatorname{tr}\left(\prod_{l=1}^{s} A_{l}^{(1)}\right)\right| \leq 2^{s} M^{s-1} \max _{1 \leq l \leq s}\left|A_{l}^{(2)}\right|_{2} .
$$

Proof. If $s=1$, the result follows from the Cauchy-Schwarz inequality. Assume $s \geq 2$. First,

$$
\mathbb{E} \circ \operatorname{tr}\left(\prod_{l=1}^{s}\left(A_{l}^{(1)}+A_{l}^{(2)}\right)\right)=\sum_{\epsilon_{1}, \ldots, \epsilon_{s} \in\{1,2\}} \mathbb{E} \circ \operatorname{tr}\left(A_{1}^{\left(\epsilon_{1}\right)} \cdots A_{s}^{\epsilon_{s}}\right) .
$$

So

$$
\left|\mathbb{E} \circ \operatorname{tr}\left(\prod_{l=1}^{s}\left(A_{l}^{(1)}+A_{l}^{(2)}\right)\right)-\mathbb{E} \circ \operatorname{tr}\left(\prod_{l=1}^{s} A_{l}^{(1)}\right)\right| \leq \sum_{\substack{\epsilon_{1}, \ldots, \epsilon_{s} \in\{1,2\} \\ \exists l_{0} \text { s.t. } \epsilon_{l_{0}} \text { is } 2}}\left|\mathbb{E} \circ \operatorname{tr}\left(A_{1}^{\left(\epsilon_{1}\right)} \cdots A_{s}^{\left(\epsilon_{s}\right)}\right)\right| .
$$


For each $\epsilon_{1}, \ldots, \epsilon_{s} \in\{1,2\}$ with $\epsilon_{l_{0}}=2$, taking $p_{l}=2(s-1)$ for $l \neq l_{0}, p_{l_{0}}=2$ and $r=1$ in Lemma 4.19, we obtain

$$
\begin{aligned}
\left|\mathbb{E} \circ \operatorname{tr}\left(A_{1}^{\left(\epsilon_{1}\right)} \cdots A_{s}^{\left(\epsilon_{s}\right)}\right)\right| & \leq\left|A_{l_{0}}^{(2)}\right|_{2}\left|A_{l_{0}+1}^{\left(\epsilon_{l_{0}+1}\right)} \cdots A_{s}^{\left(\epsilon_{s}\right)} A_{1}^{\left(\epsilon_{1}\right)} \cdots A_{l_{0}-1}^{\left(\epsilon_{l_{0}-1}\right)}\right|_{2} \\
& \leq\left|A_{l_{0}}^{(2)}\right|_{2} \prod_{j \neq l_{0}}\left|A_{j}^{\left(\epsilon_{j}\right)}\right|_{2(s-1)} \leq M^{s-1}\left|A_{l_{0}}^{(2)}\right|_{2}
\end{aligned}
$$

where for the first inequality we used the trace property and the Cauchy-Schwarz inequality, while for the second we used Hölder's inequality (Lemma 4.20). Since there are $2^{s}-1$ terms in the summation in (4.12), the desired upper bound holds.

We will now show that the off-diagonal entries of alternating products in $X_{N}$ and $X_{N}^{*}$, with deterministic diagonal matrices interspersed, have expectations that are zero or are asymptotically small as the matrix size goes to infinity.

Lemma 4.22. Let $\zeta_{1}, \ldots, \zeta_{N}$ be independent random variables uniformly distributed on the unit circle. Let $i(1), \ldots, i(r) \in\{1, \ldots, N\}$. If $j(1), \ldots, j(r) \in \mathbb{Z}$ satisfy

$$
\mathbb{E} \zeta_{i(1)}^{j(1)} \cdots \zeta_{i(r)}^{j(r)} \neq 0
$$

then $j(1)+\cdots+j(r)=0$.

Proof. Let $\pi=\operatorname{ker} i$. By Lemma 4.5, $\sum_{k \in S} j(k)=0$ for all $S \in \pi$. So

$$
\sum_{k=1}^{r} j(k)=\sum_{S \in \pi} \sum_{k \in S} j(k)=0 .
$$

Lemma 4.23. Let $n \geq 1$ be an odd number. Let $\epsilon_{1}, \ldots, \epsilon_{n} \in\{1, *\}$ be alternating. 
Let $d_{1}, \ldots, d_{n}$ be deterministic diagonal $N \times N$ matrices. Then

$$
\mathbb{E} \prod_{k=1}^{n} d_{k} X_{N}^{\epsilon_{k}}=0
$$

Proof. The proof when $\epsilon_{1}=1$ and the proof when $\epsilon_{1}=*$ are similar. So we only do the case when $\epsilon_{1}=1$. Let $i(1), i(n+1) \in\{1, \ldots, N\}$.

$$
\begin{aligned}
\left(\mathbb{E} \prod_{k=1}^{n} d_{k} X_{N}^{\epsilon_{k}}\right)_{i(1), i(n+1)} & =\sum_{i:\{2,3, \ldots, n\} \rightarrow\{1, \ldots, N\}} \mathbb{E} \prod_{k=1}^{n}\left(d_{k}\right)_{i(k), i(k)}\left(X_{N}^{\epsilon_{k}}\right)_{i(k), i(k+1)} \\
= & \sum_{\substack{i:\{2,3, \ldots, n\} \rightarrow\{1, \ldots, N\} \\
\prod_{k=1}^{n}\left(d_{k}\right)_{i(k), i(k)}}} \prod_{\mathbb{E} \prod_{k=1}^{n}\left(X_{N}^{\epsilon_{k}}\right)_{i(k), i(k+1)}}
\end{aligned}
$$

Since $\epsilon_{1}=1$ and $\epsilon_{1}, \ldots, \epsilon_{n}$ are alternating, $\epsilon_{k}=1$ when $k$ is odd, and $\epsilon_{k}=*$ when $k$ is even so

$$
\begin{aligned}
\mathbb{E} \prod_{k=1}^{n}\left(X_{N}^{\epsilon_{k}}\right)_{i(k), i(k+1)} & =\frac{1}{N^{\frac{n}{2}}} \mathbb{E} \prod_{l=1}^{(n+1) / 2}\left(X_{N}^{\epsilon_{2 l-1}}\right)_{i(2 l-1), i(2 l)} \prod_{m=1}^{(n-1) / 2}\left(X_{N}^{\epsilon_{2 m}}\right)_{i(2 m), i(2 m+1)} \\
& =\frac{1}{N^{\frac{n}{2}}} \mathbb{E} \prod_{l=1}^{(n+1) / 2} \zeta_{i(2 l-1)}^{i(2 l)} \prod_{m=1}^{(n-1) / 2} \zeta_{i(2 m+1)}^{-i(2 m)}
\end{aligned}
$$

Since the sum of the exponents is

$$
\sum_{l=1}^{(n+1) / 2} i(2 l)+\sum_{m=1}^{(n-1) / 2}(-i(2 m))=i(n+1) \neq 0
$$

by Lemma $4.23, \mathbb{E} \prod_{k=1}^{n}\left(X_{N}^{\epsilon_{k}}\right)_{i(k), i(k+1)}=0$. Thus, the result follows.

Using an argument similar to that in the proof of Lemma 4.23, one obtains

Lemma 4.24. Let $n \geq 2$ be an even number. Let $\epsilon_{1}, \ldots, \epsilon_{n} \in\{1, *\}$ be alternating. 
Let $d_{1}, \ldots, d_{n}$ be deterministic diagonal $N \times N$ matrices. If $\epsilon_{1}=*$ then

$$
\mathbb{E} \prod_{k=1}^{n} d_{k} X_{N}^{\epsilon_{k}}
$$

is a diagonal matrix.

Lemma 4.25. Let $n \geq 1$ be an even number. Let $\epsilon_{1}, \ldots, \epsilon_{n} \in\{1, *\}$ be alternating. Suppose that $\epsilon_{1}=1$. Let $d_{1}, \ldots, d_{n}$ be deterministic diagonal $N \times N$ matrices of norm at most 1. Let

$$
Z_{N}=\prod_{k=1}^{n} d_{k} X_{N}^{\epsilon_{k}}
$$

Then for every integer $p \geq 1$, there is a constant $C=C(n, p)$ such that

$$
\operatorname{Tr}\left(\left(\mathbb{E} Z_{N}-\mathbb{E} \circ \operatorname{diag} Z_{N}\right)^{*}\left(\mathbb{E} Z_{N}-\mathbb{E} \circ \operatorname{diag} Z_{N}\right)\right)^{p} \leq C .
$$

Proof. Let $i(1) \neq i(n+1) \in\{1, \ldots, N\}$. By (4.13),

$$
\begin{aligned}
\left(\mathbb{E} Z_{N}\right)_{i(1), i(n+1)} & =\left(\mathbb{E} \prod_{k=1}^{n} d_{k} X_{N}^{\epsilon_{k}}\right)_{i(1), i(n+1)} \\
& =\sum_{i:\{2,3, \ldots, n\} \rightarrow\{1, \ldots, N\}} \prod_{k=1}^{n}\left(d_{k}\right)_{i(k), i(k)} \mathbb{E} \prod_{k=1}^{n}\left(X_{N}^{\epsilon_{k}}\right)_{i(k), i(k+1)} .
\end{aligned}
$$

Since the $d_{k}$ have norms at most 1 , we have

$$
\left|\left(\mathbb{E} Z_{N}\right)_{i(1), i(n+1)}\right| \leq \sum_{i:\{2,3, \ldots, n\} \rightarrow\{1, \ldots, N\}}\left|\mathbb{E} \prod_{k=1}^{n}\left(X_{N}^{\epsilon_{k}}\right)_{i(k), i(k+1)}\right|
$$


Since $\epsilon_{k}=1$ when $k$ is odd and $\epsilon_{k}=*$ when $k$ is even,

$$
\begin{gathered}
\mathbb{E} \prod_{k=1}^{n}\left(X_{N}^{\epsilon_{k}}\right)_{i(k), i(k+1)}=\mathbb{E} \prod_{l=1}^{n / 2}\left(X_{N}^{\epsilon_{i(2 l-1)}}\right)_{i(2 l-1), i(2 l)} \prod_{m=1}^{n / 2}\left(X_{N}^{\epsilon_{2} m}\right)_{i(2 m), i(2 m+1)} \\
=\frac{1}{N^{\frac{n}{2}}} \mathbb{E} \prod_{l=1}^{n / 2} \zeta_{i(2 l-1)}^{i(2 l)} \prod_{m=1}^{n / 2} \zeta_{i(2 m+1)}^{-i(2 m)}=\frac{1}{N^{\frac{n}{2}}} \mathbb{E} \prod_{l=1}^{n / 2} \zeta_{i(2 l-1)}^{i(2 l)} \prod_{m=2}^{(n / 2)+1} \zeta_{i(2 m-1)}^{-i(2 m-2)} \\
=\frac{1}{N^{\frac{n}{2}}} \mathbb{E} \zeta_{i(1)}^{i(2)}\left(\prod_{l=2}^{n / 2} \zeta_{i(2 l-1)}^{i(2 l)}\right)\left(\prod_{m=2}^{n / 2} \zeta_{i(2 m-1)}^{-i(2 m-2)}\right) \zeta_{i(n+1)}^{-i(n)} \\
=\frac{1}{N^{\frac{n}{2}}} \mathbb{E} \zeta_{i(1)}^{i(2)}\left(\prod_{l=2}^{n / 2} \zeta_{i(2 l-1)}^{i(2 l)-i(2 l-2)}\right) \zeta_{i(n+1)}^{-i(n)} .
\end{gathered}
$$

Let $v_{1}, v_{3}, \ldots, v_{n+1} \in \mathbb{R}^{n}$ be given by

$$
\begin{aligned}
v_{1} & =e_{2} \\
v_{2 l-1} & =e_{2 l}-e_{2 l-2}, \quad\left(l=2, \ldots, \frac{n}{2}\right) \\
v_{n+1} & =-e_{n} .
\end{aligned}
$$

Let $j:\{2,4, \ldots, n\} \rightarrow\{1, \ldots, N\}$ be the restriction of $i$ to $\{2,4 \ldots, n\}$. Then we have

$$
\mathbb{E} \prod_{k=1}^{n}\left(X_{N}^{\epsilon_{k}}\right)_{i(k), i(k+1)}=\frac{1}{N^{\frac{n}{2}}} \mathbb{E} \prod_{l=1}^{(n / 2)+1} \zeta_{i(2 l-1)}^{j \cdot v_{2 l-1}}=\frac{1}{N^{\frac{n}{2}}} \mathbb{E} \prod_{k \in\{1,3, \ldots, n+1\}} \zeta_{i(k)}^{j \cdot v_{k}}
$$

Let $\pi$ be a partition of $\{1,3, \ldots, n+1\}$. Suppose that $\operatorname{ker}\left(i \uparrow_{\{1,3, \ldots, n+1\}}\right)=\pi$. For each $S \in \pi$, all the $i(k)$ are same for $k \in S$ and we denote this value by $i(S)$. Thus,

$$
\mathbb{E} \prod_{k=1}^{n}\left(X_{N}^{\epsilon_{k}}\right)_{i(k), i(k+1)}=\frac{1}{N^{\frac{n}{2}}} \mathbb{E} \prod_{S \in \pi} \zeta_{i(S)}^{j \cdot\left(\sum_{k \in S} v_{k}\right)}
$$


By Lemma 4.8,

$$
\sum_{j:\{2,4, \ldots, n\} \rightarrow\{1, \ldots, N\}}\left|\mathbb{E} \prod_{k=1}^{n}\left(X_{N}^{\epsilon_{k}}\right)_{i(k), i(k+1)}\right| \leq \frac{1}{N^{\frac{n}{2}}} N^{\frac{n}{2}-\operatorname{dim} \operatorname{span}\left\{\sum_{k \in S} v_{k}: S \in \pi\right\}}
$$

If $a_{1}, a_{3}, \ldots, a_{n+1}$ are scalars satisfying

$$
a_{1} v_{1}+a_{3} v_{3}+\ldots+a_{n+1} v_{n+1}=0
$$

then $a_{1}=a_{3}=\ldots=a_{n+1}$. Thus, by Lemma $4.9, \operatorname{dim} \operatorname{span}\left\{\sum_{k \in S} v_{k}: S \in \pi\right\} \geq$ $|\pi|-1$, so

$$
\begin{aligned}
\sum_{j:\{2,4, \ldots, n\} \rightarrow\{1, \ldots, N\}}\left|\mathbb{E} \prod_{k=1}^{n}\left(X_{N}^{\epsilon_{k}}\right)_{i(k), i(k+1)}\right| & \leq \frac{1}{N^{\frac{n}{2}}} N^{\frac{n}{2}-(|\pi|-1)} \\
& =N^{1-|\pi|}
\end{aligned}
$$

Considering all the cases when $\{1\}$ is or is not a singleton block and $\{n+1\}$ is or is not a singleton block of $\pi$, we see that the number of choices of $i(3), i(5), \ldots, i(n-1)$ such that $\operatorname{ker}\left(i\left\lceil_{\{1,3, \ldots, n+1\}}\right)=\pi\right.$ is at most $N^{|\pi|-2}$. Thus,

$$
\sum_{\substack{i:\{2, \ldots, n\} \rightarrow\{1, \ldots, N\} \\ \operatorname{ker}\left(i \uparrow_{\{1,3, \ldots, n+1\})=\pi}\right.}}\left|\mathbb{E} \prod_{k=1}^{n}\left(X_{N}^{\epsilon_{k}}\right)_{i(k), i(k+1)}\right| \leq N^{|\pi|-2} N^{1-|\pi|}=\frac{1}{N}
$$

Summing over all partitions $\pi$ of $\{1,3, \ldots, n+1\}$, we have

$$
\sum_{i:\{2, \ldots, n\} \rightarrow\{1, \ldots, N\}}\left|\mathbb{E} \prod_{k=1}^{n}\left(X_{N}^{\epsilon_{k}}\right)_{i(k), i(k+1)}\right| \leq \frac{C_{n}}{N}
$$

So by (4.15),

$$
\left|\left(\mathbb{E} Z_{N}\right)_{i(1), i(n+1)}\right| \leq \frac{C_{n}}{N}
$$


So each entry in $\mathbb{E}\left(Z_{N}-\operatorname{diag} Z_{N}\right)$ has absolute value at most $C_{n} / N$. From this, the result follows easily. Indeed, each entry of

$$
\left(\mathbb{E} Z_{N}-\mathbb{E} \circ \operatorname{diag} Z_{N}\right)^{*}\left(\mathbb{E} Z_{N}-\mathbb{E} \circ \operatorname{diag} Z_{N}\right)
$$

has absolute value at most $C_{n}^{2} / N$. Taken to the $p$-th power, every entry has absolute value at most $C_{n}^{2 p} / N$, and the result (4.14) follows with constant $C=C_{n}^{2 p}$.

Using a similar argument as in the proof of Lemma 4.25, (essentially, by treating also the case $i(n+1)=i(1)$ in that proof) one obtains the following lemma.

Lemma 4.26 (Compare with Proposition 1 in [30]). For every integer $p \geq 1$, we have $\mathbb{E} \circ \operatorname{Tr}\left(X_{N}^{*} X_{N}\right)^{p} \leq C N$, where $C$ depends only on $p$.

Lemma 4.27. Let $\epsilon_{1}, \ldots, \epsilon_{n} \in\{1, *\}$ be alternating. Let $d_{1}, \ldots, d_{n}$ be deterministic diagonal $N \times N$ matrices of norm at most 1 . Let

$$
Z_{N}=\prod_{k=1}^{n} d_{k} X_{N}^{\epsilon_{k}}
$$

Then for every integer $p \geq 1$,

$$
\left|Z_{N}\right|_{2 p} \leq C \quad \text { and } \quad\left|\mathbb{E} Z_{N}-\mathbb{E} \circ \operatorname{diag} Z_{N}\right|_{2 p} \leq \frac{C}{N^{\frac{1}{2 p}}}
$$

where $C$ depends only on $n$ and $p$.

Proof. By Lemma 4.26, for every integer $q \geq 1$,

$$
\left|X_{N}\right|_{2 q} \leq C_{q}
$$

where $C_{q}$ depends only on $q$. Thus, taking $p_{1}=\cdots=p_{n}=2 p n$ and $r=2 p$ in 
Lemma 4.20, we have

$$
\begin{aligned}
\left|Z_{N}\right|_{2 p}=\left|\prod_{k=1}^{n} d_{k} X_{N}^{\epsilon_{k}}\right|_{2 p} & \leq \prod_{k=1}^{n}\left|d_{k} X_{N}^{\epsilon_{k}}\right|_{2 p n} \\
& \leq \prod_{k=1}^{n}\left\|d_{k}\right\|\left|X_{N}^{\epsilon_{k}}\right|_{2 p n} \leq \prod_{k=1}^{n}\left|X_{N}^{\epsilon_{k}}\right|_{2 p n} \leq \prod_{k=1}^{n} C_{2 p n}
\end{aligned}
$$

This proves the first inequality. The other inequality follows by combining Lemmas 4.23, 4.24 and 4.25 .

We are now ready to prove Proposition 4.2. We first prove a weaker version of it, with $\mathbb{E} \circ$ diag replaced by $\mathbb{E}$. The convention regarding ordering in products is described in Definition 4.1.2.

Lemma 4.28. Let $n \geq 1$. Let $\epsilon_{1}, \ldots, \epsilon_{n} \in\{1, *\}$. Let $\sigma$ be the interval partition of $\{1, \ldots, n\}$ defined by

$$
k \stackrel{\sigma}{\sim} k+1 \Longleftrightarrow \epsilon_{k} \neq \epsilon_{k+1}
$$

for $k \in\{1, \ldots, n-1\}$. Let $d_{1}, \ldots, d_{n}$ be deterministic diagonal $N \times N$ matrices of norm at most 1 . Then

$$
\left|\mathbb{E} \circ \operatorname{Tr} \prod_{S \in \sigma}\left(\prod_{k \in S} d_{k} X_{N}^{\epsilon_{k}}-\mathbb{E}\left(\prod_{k \in S} d_{k} X_{N}^{\epsilon_{k}}\right)\right)\right| \leq C \sqrt{N},
$$

where $C$ depends only on $n$. 
Proof. Since $\sigma$ is an interval partition, we can expand

$$
\begin{aligned}
& \mathbb{E} \circ \operatorname{Tr} \prod_{S \in \sigma}\left(\prod_{k \in S} d_{k} X_{N}^{\epsilon_{k}}-\mathbb{E}\left(\prod_{k \in S} d_{k} X_{N}^{\epsilon_{k}}\right)\right) \\
&= \sum_{\substack{i:\{1, \ldots, n+1\} \rightarrow\{1, \ldots, N\} \\
i(n+1)=i(1)}}\left(\mathbb { E } \prod _ { S \in \sigma } \left(\prod_{k \in S}\left(d_{k}\right)_{i(k), i(k)}\left(X_{N}^{\epsilon_{k}}\right)_{i(k), i(k+1)}\right.\right. \\
&=\left.\left.-\mathbb{E}\left(\prod_{k \in S}\left(d_{k}\right)_{i(k), i(k)}\left(X_{N}^{\epsilon_{k}}\right)_{i(k), i(k+1)}\right)\right)\right) \\
& \sum_{\substack{i:\{1, \ldots, n+1\} \rightarrow\{1, \ldots, N\} \\
i(n+1)=i(1)}} \prod_{k=1}^{n}\left(d_{k}\right)_{i(k), i(k)}\left(\mathbb { E } \prod _ { S \in \sigma } \left(\prod_{k \in S}\left(X_{N}^{\epsilon_{k}}\right)_{i(k), i(k+1)}\right.\right. \\
&\left.\quad-\mathbb{E}\left(\prod_{k \in S}\left(X_{N}^{\epsilon_{k}}\right)_{i(k), i(k+1)}\right)\right) .
\end{aligned}
$$

Since the $d_{k}$ have norms at most 1 , it follows that

$$
\begin{aligned}
\left|\mathbb{E} \circ \operatorname{Tr} \prod_{S \in \sigma}\left(\prod_{k \in S} d_{k} X_{N}^{\epsilon_{k}}-\mathbb{E}\left(\prod_{k \in S} d_{k} X_{N}^{\epsilon_{k}}\right)\right)\right| & \\
\leq \sum_{i:\{1, \ldots, n+1\} \rightarrow\{1, \ldots, N\}} \delta_{i(n+1), i(1)} \mid \mathbb{E} & \prod_{S \in \sigma}\left(\prod_{k \in S}\left(X_{N}^{\epsilon_{k}}\right)_{i(k), i(k+1)}\right. \\
& \left.-\mathbb{E}\left(\prod_{k \in S}\left(X_{N}^{\epsilon_{k}}\right)_{i(k), i(k+1)}\right)\right) \mid .
\end{aligned}
$$

For each $i:\{1, \ldots, n+1\} \rightarrow\{1, \ldots, N\}$, by Lemma 4.6, we have

$$
\begin{aligned}
\left|\mathbb{E} \prod_{S \in \sigma}\left(\prod_{k \in S}\left(X_{N}^{\epsilon_{k}}\right)_{i(k), i(k+1)}-\mathbb{E}\left(\prod_{k \in S}\left(X_{N}^{\epsilon_{k}}\right)_{i(k), i(k+1)}\right)\right)\right| \\
\leq \mathbb{E} \prod_{S \in \sigma}\left(\prod_{k \in S}\left(X_{N}^{\epsilon_{k}}\right)_{i(k), i(k+1)}\right)=\mathbb{E} \prod_{k=1}^{n}\left(X_{N}^{\epsilon_{k}}\right)_{i(k), i(k+1)} .
\end{aligned}
$$

Let

$$
U=\left\{2 \leq k \leq n: \epsilon_{k-1}=1 \text { and } \epsilon_{k}=*\right\} .
$$


Let $Y_{N}=\sqrt{N} X_{N}$. Then

$$
\begin{aligned}
\mathbb{E} \prod_{k=1}^{n}\left(Y_{N}^{\epsilon_{k}}\right)_{i(k), i(k+1)} & =\mathbb{E} \prod_{k \in U}\left(Y_{N}^{\epsilon_{k}}\right)_{i(k), i(k+1)} \prod_{k \in U-1}\left(Y_{N}^{\epsilon_{k}}\right)_{i(k), i(k+1)} \prod_{k \in\{1, \ldots, n\} \backslash(U \cup(U-1))}\left(Y_{N}^{\epsilon_{k}}\right)_{i(k), i(k+1)} \\
= & \mathbb{E} \prod_{k \in U}\left(Y_{N}^{\epsilon_{k}}\right)_{i(k), i(k+1)} \prod_{k \in U}\left(Y_{N}^{\epsilon_{k-1}}\right)_{i(k-1), i(k)} \prod_{k \in\{1, \ldots, n\} \backslash(U \cup(U-1))}\left(Y_{N}^{\epsilon_{k}}\right)_{i(k), i(k+1)} \\
= & \mathbb{E} \prod_{k \in U} \zeta_{i(k+1)}^{-i(k)} \prod_{k \in U} \zeta_{i(k-1)}^{i(k)} \prod_{k \in\{1, \ldots, n\} \backslash(U \cup(U-1))}\left(Y_{N}^{\epsilon_{k}}\right)_{i(k), i(k+1)} \\
= & \mathbb{E} \prod_{k \in U+1} \zeta_{i(k)}^{-i(k-1)} \prod_{k \in U-1} \zeta_{i(k)}^{i(k+1)} \prod_{k \in\{1, \ldots, n\} \backslash(U \cup(U-1))}\left(Y_{N}^{\epsilon_{k}}\right)_{i(k), i(k+1)} \\
= & \left.\mathbb{E} \prod_{k \in(U+1) \backslash(U-1)} \zeta_{i(k)}^{-i(k-1)} \prod_{(U-1) \backslash(U+1)} \zeta_{i(k)}^{i(k+1)} \prod_{k \in(U+1) \cap(U-1)} \zeta_{i(k)}^{i(k+1)-i(k-1)} \prod_{k \in\{1, \ldots, n\} \backslash(U \cup(U-1))}\left(Y_{N}^{\epsilon_{k}}\right)_{i(k), i(k+1)}\right)
\end{aligned}
$$

Let $L=\{1, \ldots, n+1\} \backslash U$. Note that by the definition of $U,(U-1) \cup(U+1) \subset L$. Also, if $k \in\{1, \ldots, n+1\} \backslash(U \cup(U-1))$ then $k \in L$ and $k+1 \in L$.

Let $i_{U}: U \rightarrow\{1, \ldots, N\}$ be the restriction of $i$ to $U$. Let $i_{L}: L \rightarrow\{1, \ldots, N\}$ be the restriction of $i$ to $L$. With $v_{k} \in \mathbf{R}^{U}$ defined as in Lemma 4.13, we have

$$
\mathbb{E} \prod_{k=1}^{n}\left(Y_{N}^{\epsilon_{k}}\right)_{i(k), i(k+1)}=\mathbb{E} \prod_{k \in(U-1) \cup(U+1)} \zeta_{i_{L}(k)}^{i_{U} \cdot v_{k}} \prod_{k \in\{1, \ldots, n\} \backslash(U \cup(U-1))}\left(Y_{N}^{\epsilon_{k}}\right)_{i_{L}(k), i_{L}(k+1)},
$$

where we think of $i_{U}$ as belonging to $\mathbf{R}^{U}$. Let $\pi$ be a partition of $L$. Suppose that ker $i_{L}=\pi$. Let $\pi_{1}=\pi \uparrow_{(U-1) \cup(U+1)}$. For each block $S \in \pi_{1}$, all the $i_{L}(k)$ are the 
same for $k \in S$ and we denote this value by $i_{L}(S)$. It follows that

$$
\begin{aligned}
\mathbb{E} \prod_{k=1}^{n}\left(Y_{N}^{\epsilon_{k}}\right)_{i(k), i(k+1)} & =\mathbb{E} \prod_{S \in \pi_{1}} \prod_{k \in S} \zeta_{i_{L}(k)}^{i_{U} \cdot v_{k}} \prod_{k \in\{1, \ldots, n\} \backslash(U \cup(U-1))}\left(Y_{N}^{\epsilon_{k}}\right)_{i_{L}(k), i_{L}(k+1)} \\
& =\mathbb{E} \prod_{S \in \pi_{1}} \zeta_{i_{L}(S)}^{i_{U} \cdot\left(\sum_{k \in S} v_{k}\right)} \prod_{k \in\{1, \ldots, n\} \backslash(U \cup(U-1))}\left(Y_{N}^{\epsilon_{k}}\right)_{i_{L}(k), i_{L}(k+1)}
\end{aligned}
$$

Note that the term $\prod_{k \in\{1, \ldots, n\} \backslash(U \cup(U-1))}\left(Y_{N}^{\epsilon_{k}}\right)_{i_{L}(k), i_{L}(k+1)}$ is a product of the random variables $\left(\zeta_{j}\right)_{j \in L}$ and their inverses, possibly with repetition. Thus by Lemma 4.8, fixing $i_{L}$ and summing over all $i_{U}$, we have

$$
\sum_{i_{U}: U \rightarrow\{1, \ldots, N\}}\left|\mathbb{E} \prod_{k=1}^{n}\left(Y_{N}^{\epsilon_{k}}\right)_{i(k), i(k+1)}\right| \leq N^{|U|-\operatorname{dim} \operatorname{span}\left\{\sum_{k \in S} v_{k}: S \in \pi_{1}\right\}}
$$

Summing now over all $i_{L}$ with $\operatorname{ker} i_{L}=\pi$, we obtain

$$
\sum_{\left\{i_{L}: \operatorname{ker} i_{L}=\pi\right\}} \sum_{i_{U}: U \rightarrow\{1, \ldots, N\}}\left|\mathbb{E} \prod_{k=1}^{n}\left(Y_{N}^{\epsilon_{k}}\right)_{i(k), i(k+1)}\right| \leq N^{|\pi|+|U|-\operatorname{dim} \operatorname{span}\left\{\sum_{k \in S} v_{k}: S \in \pi_{1}\right\}}
$$

Let $\sim$ be the equivalence relation on $(U-1) \cup(U+1)$ generated by $l-1 \sim l+1$ $\forall l \in U$. Let $\pi_{2}$ be the partition of $(U-1) \cup(U+1)$ that corresponds to $\sim$. By Lemma 4.13 and Lemma 4.9,

$$
\operatorname{dim} \operatorname{span}\left\{\sum_{k \in S} v_{k}: S \in \pi_{1}\right\} \geq\left|\pi_{1}\right|-\left|\pi_{1} \vee \pi_{2}\right|
$$


Thus,

$$
\begin{aligned}
|\pi|-\operatorname{dim} \operatorname{span}\{ & \left.\sum_{k \in S} v_{k}: S \in \pi_{1}\right\} \leq|\pi|-\left|\pi_{1}\right|+\left|\pi_{1} \vee \pi_{2}\right| \\
& =|\pi|-\mid \pi\left\lceil( U - 1 ) \cup ( U + 1 ) | + | \left(\pi\lceil(U-1) \cup(U+1)) \vee \pi_{2} \mid\right.\right. \\
& =\left|\pi \vee\left(\pi_{2} \cup\{\{l\}: l \in L \backslash((U-1) \cup(U+1))\}\right)\right|,
\end{aligned}
$$

where the last equation follows from Lemma 4.10 by taking $K=(U-1) \cup(U+1)$ and $\lambda=\pi_{2}$.

Case I: $\left|\pi \vee\left(\pi_{2} \cup\{\{l\}: l \in L \backslash((U-1) \cup(U+1))\}\right)\right| \leq \frac{1}{2}(n+1-2|U|)$.

In this case, by (4.19),

$$
|\pi|-\operatorname{dim} \operatorname{span}\left\{\sum_{k \in S} v_{k}: S \in \pi_{1}\right\} \leq \frac{1}{2}(n+1-2|U|)
$$

Thus, by (4.18),

$$
\sum_{\operatorname{ker} i_{L}=\pi} \sum_{i_{U}: U \rightarrow\{1, \ldots, N\}}\left|\mathbb{E} \prod_{k=1}^{n}\left(Y_{N}^{\epsilon_{k}}\right)_{i(k), i(k+1)}\right| \leq N^{|U|+\frac{1}{2}(n+1-2|U|)}
$$

Since $X_{N}=\frac{1}{\sqrt{N}} Y_{N}$,

$$
\sum_{\operatorname{ker} i_{L}=\pi} \sum_{i_{U}: U \rightarrow\{1, \ldots, N\}}\left|\mathbb{E} \prod_{k=1}^{n}\left(X_{N}^{\epsilon_{k}}\right)_{i(k), i(k+1)}\right| \leq N^{|U|+\frac{1}{2}(n+1-2|U|)-\frac{n}{2}}=\sqrt{N}
$$

By (4.17),

$$
\sum_{\operatorname{ker} i_{L}=\pi} \sum_{i_{U}: U \rightarrow\{1, \ldots, N\}}\left|\mathbb{E} \prod_{S \in \sigma}\left(\prod_{k \in S}\left(X_{N}^{\epsilon_{k}}\right)_{i(k), i(k+1)}-\mathbb{E}\left(\prod_{k \in S}\left(X_{N}^{\epsilon_{k}}\right)_{i(k), i(k+1)}\right)\right)\right| \leq \sqrt{N} .
$$

Case II: $\left|\pi \vee\left(\pi_{2} \cup\{\{l\}: l \in L \backslash((U-1) \cup(U+1))\}\right)\right|>\frac{1}{2}(n+1-2|U|)$. 
By Lemma 4.14, $\left|\pi_{2}\right| \leq|(U+1) \backslash(U-1)|$ so

$$
\begin{aligned}
\mid \pi_{2} \cup\{\{l\}: l \in L \backslash((U-1) & \cup(U+1))\}|=| \pi_{2}|+| L \backslash((U-1) \cup(U+1)) \mid \\
& \leq|(U+1) \backslash(U-1)|+|L|-|(U-1) \cup(U+1)| \\
& =|L|-|U-1|=|L|-|U|=n+1-2|U| .
\end{aligned}
$$

By Lemma 4.12, $\pi_{2} \cup\{\{l\}: l \in L \backslash((U-1) \cup(U+1))\}$ contains a block $S_{1}$ such that

$$
\left\{i_{L}(l): l \in S_{1}\right\} \cap\left\{i_{L}(l): l \in L \backslash S_{1}\right\}=\emptyset .
$$

If $S_{1} \in \pi_{2}$ then by Lemma $4.16(\mathrm{i})$,

$$
S_{1}=L\left(S_{0}\right)=\left\{k \in S_{0}: \epsilon_{k}=1\right\} \cup\left\{k+1: k \in S_{0} \text { and } \epsilon_{k}=*\right\}
$$

for some $S_{0} \in \sigma$. If $S_{1}=\{l\}$ for some $l \in L \backslash((U-1) \cup(U+1))$ and $l \neq 1, n+1$, then by Lemma $4.16(\mathrm{ii}), S_{1}$ is also the form (4.21). If $S_{1}=\{1\}$ or $\{n+1\}$ then since 1 and $n+1$ are both in $L$ (by the definition of $U$ ), it follows from (4.20) that $i_{L}(1) \neq i_{L}(n+1)$ and so

$$
\begin{array}{r}
\sum_{\operatorname{ker} i_{L}=\pi} \sum_{i_{U}: U \rightarrow\{1, \ldots, N\}} \delta_{i(n+1), i(1)} \mid \mathbb{E} \prod_{S \in \sigma}\left(\prod_{k \in S}\left(X_{N}^{\epsilon_{k}}\right)_{i(k), i(k+1)}\right. \\
\left.\quad-\mathbb{E}\left(\prod_{k \in S}\left(X_{N}^{\epsilon_{k}}\right)_{i(k), i(k+1)}\right)\right) \mid=0 .
\end{array}
$$

If $S_{1}$ is of the form (4.21) then by $(4.20)$,

$$
\left\{i_{L}(l): l \in L\left(S_{0}\right)\right\} \cap\left\{i_{L}(l): l \in L \backslash L\left(S_{0}\right)\right\}=\emptyset
$$


By Lemma 4.15, $\cup_{S \neq S_{0}} L(S) \subset L \backslash L\left(S_{0}\right)$. So

$$
\left\{i_{L}(l): l \in L\left(S_{0}\right)\right\} \cap\left\{i_{L}(l): l \in \cup_{S \neq S_{0}} L(S)\right\}=\emptyset .
$$

Note that for each $S \in \sigma, \prod_{k \in S}\left(X_{N}^{\epsilon_{k}}\right)_{i(k), i(k+1)}$ depends only on $\left\{\zeta_{i_{L}(l)}: l \in L(S)\right\}$. Thus, the random variable

$$
\prod_{k \in S_{0}}\left(X_{N}^{\epsilon_{k}}\right)_{i(k), i(k+1)}
$$

is independent of the random variables

$$
\prod_{k \in S}\left(X_{N}^{\epsilon_{k}}\right)_{i(k), i(k+1)}, \quad S \neq S_{0}
$$

Thus,

$$
\left|\mathbb{E} \prod_{S \in \sigma}\left(\prod_{k \in S}\left(X_{N}^{\epsilon_{k}}\right)_{i(k), i(k+1)}-\mathbb{E}\left(\prod_{k \in S}\left(X_{N}^{\epsilon_{k}}\right)_{i(k), i(k+1)}\right)\right)\right|=0 .
$$

So again (4.22) holds.

Combining the conclusions of Case I and Case II and summing over all partitions $\pi$ of $L$, we get

$$
\begin{array}{r}
\sum_{i_{L}: L \rightarrow\{1, \ldots, N\}} \sum_{i_{U}: U \rightarrow\{1, \ldots, N\}} \delta_{i(n+1), i(1)} \mid \mathbb{E} \prod_{S \in \sigma}\left(\prod_{k \in S}\left(X_{N}^{\epsilon_{k}}\right)_{i(k), i(k+1)}\right. \\
\left.-\mathbb{E}\left(\prod_{k \in S}\left(X_{N}^{\epsilon_{k}}\right)_{i(k), i(k+1)}\right)\right) \mid \leq C \sqrt{N},
\end{array}
$$

where $C$ is the number of partitions of $L$. By (4.16), the result follows.

Proof of Proposition 4.2. Let

$$
Z_{N, I}=\prod_{k \in I} d_{k} X_{N}^{\epsilon_{k}}
$$


By Lemma 4.27 and Lemma 4.17, for every integer $p \geq 1$,

$$
\left|Z_{N, I}-\mathbb{E} Z_{N, I}\right|_{2 p} \leq C
$$

and

$$
\left|\mathbb{E} Z_{N, I}-\mathbb{E} \circ \operatorname{diag} Z_{N, I}\right|_{2 p} \leq C
$$

where $C$ depends only on $n$ and $p$. So by Lemma 4.21 ,

$$
\begin{aligned}
& \left|\mathbb{E} \circ \operatorname{tr} \prod_{I \in \sigma}\left(Z_{N, I}-\mathbb{E} Z_{N, I}\right)-\mathbb{E} \circ \operatorname{tr} \prod_{I \in \sigma}\left(Z_{N, I}-\mathbb{E} \circ \operatorname{diag} Z_{N, I}\right)\right| \\
\leq & \sup _{I \in \sigma}\left|\mathbb{E} Z_{N, I}-\mathbb{E} \circ \operatorname{diag} Z_{N, I}\right|_{2},
\end{aligned}
$$

where $C$ depends only on $n$. By Lemma 4.27 for $p=1$,

$$
\left|\mathbb{E} Z_{N, I}-\mathbb{E} \circ \operatorname{diag} Z_{N, I}\right|_{2} \leq \frac{C}{\sqrt{N}}, \quad I \in \sigma
$$

Therefore,

$$
\left|\mathbb{E} \circ \operatorname{tr} \prod_{I \in \sigma}\left(Z_{N, I}-\mathbb{E} Z_{N, I}\right)-\mathbb{E} \circ \operatorname{tr} \prod_{I \in \sigma}\left(Z_{N, I}-\mathbb{E} \circ \operatorname{diag} Z_{N, I}\right)\right| \leq \frac{C}{\sqrt{N}}
$$

Thus, by Lemma 4.28, the result follows.

We are now ready to prove the main result. For a $\mathrm{C}^{*}$-algebra $B$, by $B\left\langle X, X^{*}\right\rangle$ we denote the $*$-algebra of polynomials in noncommuting variables $X$ and $X^{*}$ with coefficients on $B$; technically this is the algebraic free product of the three algebras $B, \mathbf{C}[X]$ and $\mathbf{C}\left[X^{*}\right]$ with amalgamation over the scalars. We endow $B\left\langle X, X^{*}\right\rangle$ with the obvious $*$-operation. 
Theorem 4.3.1. Consider the $\mathrm{C}^{*}$-algebra $B=C[0,1]$ with tracial state $\tau: B \rightarrow \mathbf{C}$ obtained by integration using Lebesgue measure. Let $\mathcal{E}: B\left\langle X, X^{*}\right\rangle \rightarrow B$ be the linear, self-adjoint, $B$-bimodular map that is the identity on $B$ and so that with respect to $\mathcal{E}, X$ is $B$-valued $\mathrm{R}$-diagonal with even alternating moments given by, for every $n \in \mathbf{N}$ and $b_{1}, \ldots, b_{2 n} \in B$,

$$
\begin{aligned}
\mathcal{E}\left(b_{1} X^{*} b_{2} X b_{3} X^{*} b_{4} X \cdots\right. & \left.b_{2 n-1} X^{*} b_{2 n} X\right) \\
& =\sum_{\pi \in \mathcal{P}(n)} b_{1} \Lambda_{\pi}\left(b_{3}, b_{5}, \ldots, b_{2 n-1}\right) \tau\left(\Gamma_{\pi}\left(b_{2}, b_{4}, \ldots, b_{2 n}\right)\right) \\
\mathcal{E}\left(b_{1} X b_{2} X^{*} b_{3} X b_{4} X^{*} \cdots b_{2 n-1} X b_{2 n} X^{*}\right) & \\
= & \sum_{\pi \in \mathcal{P}(n)} \Gamma_{\pi}\left(b_{1}, b_{3}, \ldots, b_{2 n-1}\right) \tau\left(\Lambda_{\pi}\left(b_{2}, b_{4}, \ldots, b_{2 n-2}\right) b_{2 n}\right) .
\end{aligned}
$$

Then for all $n \in \mathbf{N}, \epsilon(1), \ldots, \epsilon(n) \in\{1, *\}$ and all $b_{1}, \ldots, b_{n} \in B$, we have

$$
\begin{aligned}
& \lim _{N \rightarrow \infty} \mathbb{E} \circ \operatorname{tr}\left(D_{N}\left(b_{1}\right) X_{N}^{\epsilon(1)} D_{N}\left(b_{2}\right) X_{N}^{\epsilon(2)} \cdots D_{N}\left(b_{n}\right) X_{N}^{\epsilon(n)}\right) \\
= & \tau \circ \mathcal{E}\left(b_{1} X^{\epsilon(1)} b_{2} X^{\epsilon(2)} \cdots b_{n} X^{\epsilon(n)}\right)
\end{aligned}
$$

where, for $b \in B, D_{N}(b)$ is the scalar diagonal matrix

$$
D_{N}(b)=\operatorname{diag}\left(b\left(\frac{1}{N}\right), b\left(\frac{2}{N}\right), \ldots, b\left(\frac{N}{N}\right)\right) .
$$

Proof. Let $n \in \mathbf{N}$ and suppose $b_{1}, \ldots, b_{n} \in N$ and $\epsilon(1), \ldots, \epsilon(n) \in\{1, *\}$ are arbitrary. We will prove (4.25) by induction on $n$. In the case of $n=1$, the right-handside of (4.25) is zero and, by Lemma 4.23, so is the left-hand-side of (4.25). For the induction step, let $\sigma=\sigma(\epsilon)$ be the maximal alternating interval partition of $\epsilon$ (see 
Definition 4.1.1). For $I \in \sigma$, let

$$
c_{I}=\mathcal{E}\left(\prod_{j \in I} b_{j} X^{\epsilon(j)}\right) \in B
$$

where the product is taken in increasing order of the index $j$. By $B$-valued Rdiagonality of $X$,

$$
\tau \circ \mathcal{E}\left(\prod_{I \in \sigma}\left(\prod_{j \in I} b_{j} X^{\epsilon(j)}-c_{I}\right)\right)=0,
$$

where the product over $I \in \sigma$ is taken in order of increasing elements of the interval blocks $I$ (since $\sigma$ is an interval partition, given two distinct blocks, all the elements of one of them are less than all the elements of the other). Expanding the above product over $I \in \sigma$, we get a sum of $2^{|\sigma|}$ terms that enables $\tau \circ \mathcal{E}\left(\prod_{j=1}^{n} b_{j} X^{\epsilon(j)}\right)$ to be expressed as $(-1)^{|\sigma|-1} \tau\left(\prod_{I \in \sigma} c_{I}\right)$ plus the sum of $2^{|\sigma|}-2$ terms, each of the form

$$
(-1)^{\left|\sigma \backslash \sigma^{\prime}\right|-1} \tau \circ \mathcal{E}\left(\prod_{J \in K} f_{j} X^{\epsilon(j)}\right)
$$

where $K$ is the union of a proper subset $\sigma^{\prime}$ of $\sigma$ and for certain $f_{j} \in B$, equal to the product of $b_{j}$ and some of $\left(c_{I}\right)_{I \in \sigma \backslash \sigma^{\prime}}$.

We will show

$$
\lim _{N \rightarrow \infty} \mathbb{E} \circ \operatorname{tr}\left(\prod_{I \in \sigma}\left(\prod_{j \in I} D_{N}\left(b_{j}\right) X_{N}^{\epsilon(j)}-D_{N}\left(c_{I}\right)\right)\right)=0 .
$$

This will prove the induction step, because expansion of the left-hand-side of (4.27) as a sum of $2^{|\sigma|}$ terms will enable

$$
\lim _{N \rightarrow \infty} \mathbb{E} \circ \operatorname{tr}\left(\prod_{j=1}^{n} D_{N}\left(b_{j}\right) X_{N}^{\epsilon(j)}\right)
$$


to be written as

$$
(-1)^{|\sigma|-1} \lim _{N \rightarrow \infty} \mathbb{E} \circ \operatorname{tr}\left(\prod_{I \in \sigma} D_{N}\left(c_{I}\right)\right)=(-1)^{|\sigma|-1} \tau\left(\prod_{I \in \sigma} c_{I}\right)
$$

plus the sum of $2^{|\sigma|-1}$ terms, each equal to

$$
(-1)^{\left|\sigma \backslash \sigma^{\prime}\right|-1} \lim _{N \rightarrow \infty} \mathbb{E} \circ \operatorname{tr}\left(\prod_{J \in K} D_{N}\left(f_{j}\right) X_{N}^{\epsilon(j)}\right),
$$

for the same $K$ and $f_{j}$ as appeared in (4.26). By the inductive hypothesis, each of the terms in (4.28) is equal to the corresponding term in (4.26). This shows that proof of the induction step will follow, once we have proven (4.27).

In order to verify (4.27), we will use Proposition 4.2, which yields

$$
\lim _{N \rightarrow \infty} \mathbb{E} \circ \operatorname{tr}\left(\prod_{I \in \sigma}\left(\prod_{j \in I} D_{N}\left(b_{j}\right) X_{N}^{\epsilon(j)}-\mathbb{E} \circ \operatorname{diag}\left(\prod_{j \in I} D_{N}\left(b_{j}\right) X_{N}^{\epsilon(j)}\right)\right)\right)=0 .
$$

For $I \in \sigma$, if $n$ is even, then from Proposition 4.1 and (4.23)-(4.24), we have

$$
\lim _{N \rightarrow \infty}\left\|\mathbb{E} \circ \operatorname{diag}\left(\prod_{j \in I} D_{N}\left(b_{j}\right) X_{N}^{\epsilon(j)}\right)-D_{N}\left(c_{I}\right)\right\|=0
$$

while if $n$ is odd, then by R-diagonality of $X$ we have $c_{I}=0$ and from Lemma 4.23, we see that also in this case (4.30) holds. We now write, for each $I \in \sigma$,

$$
\begin{array}{r}
\prod_{j \in I} D_{N}\left(b_{j}\right) X_{N}^{\epsilon(j)}-D_{N}\left(c_{I}\right)=\left(\prod_{j \in I} D_{N}\left(b_{j}\right) X_{N}^{\epsilon(j)}-\mathbb{E} \circ \operatorname{diag}\left(\prod_{j \in I} D_{N}\left(b_{j}\right) X_{N}^{\epsilon(j)}\right)\right) \\
+\left(\mathbb{E} \circ \operatorname{diag}\left(\prod_{j \in I} D_{N}\left(b_{j}\right) X_{N}^{\epsilon(j)}\right)-D_{N}\left(c_{I}\right)\right)
\end{array}
$$

and, in the left-hand-side of (4.27), distribute, resulting in a sum of $2^{|\sigma|}$ limits, each 
of which will be seen to equal 0 . That the first of these limits is zero is precisely the import of (4.29). That each of the other limits is zero is a consquence of (4.30) and Hölder's inequality, (see, Lemma 4.20). Indeed, each of the other limits is of the form

$$
\lim _{N \rightarrow \infty} \mathbb{E} \circ \operatorname{tr}\left(\prod_{I \in \sigma} F_{I}\right)
$$

where $F_{I}$ is either

$$
\left(\prod_{j \in I} D_{N}\left(b_{j}\right) X_{N}^{\epsilon(j)}-\mathbb{E} \circ \operatorname{diag}\left(\prod_{j \in I} D_{N}\left(b_{j}\right) X_{N}^{\epsilon(j)}\right)\right)
$$

or

$$
\left(\mathbb{E} \circ \operatorname{diag}\left(\prod_{j \in I} D_{N}\left(b_{j}\right) X_{N}^{\epsilon(j)}\right)-D_{N}\left(c_{I}\right)\right)
$$

and for at least one $I \in \sigma$ it is the latter. Now from (4.30), we conclude that, for every $I \in \sigma$,

$$
\left\|\mathbb{E} \circ \operatorname{diag}\left(\prod_{j \in I} D_{N}\left(b_{j}\right) X_{N}^{\epsilon(j)}\right)\right\|
$$

remains bounded as $N \rightarrow \infty$. From Lemma 4.27, we have that, for every $I \in \sigma$ and every integer $p \geq 1$,

$$
\left|\prod_{j \in I} D_{N}\left(b_{j}\right) X_{N}^{\epsilon(j)}\right|_{2 p}
$$

remains bounded as $N \rightarrow \infty$. Consequently, for every $I \in \sigma$,

$$
\left|\prod_{j \in I} D_{N}\left(b_{j}\right) X_{N}^{\epsilon(j)}-\mathbb{E} \circ \operatorname{diag}\left(\prod_{j \in I} D_{N}\left(b_{j}\right) X_{N}^{\epsilon(j)}\right)\right|_{2 p}
$$


remains bounded as $N \rightarrow \infty$. Of course, from (4.30) we get, for every $I$ and $p$,

$$
\lim _{N \rightarrow \infty}\left|\mathbb{E} \circ \operatorname{diag}\left(\prod_{j \in I} D_{N}\left(b_{j}\right) X_{N}^{\epsilon(j)}\right)-D_{N}\left(c_{I}\right)\right|_{2 p}=0
$$

Consequently, taking $p=|\sigma|$ and applying Hölder's inequality, we get that for every product $\prod_{I \in \sigma} F_{I}$ of the form described at (4.31),

$$
\lim _{N \rightarrow \infty}\left|\prod_{I \in \sigma} F_{I}\right|_{2}=0
$$

Now using the Cauchy-Schwarz inequality, we conclude

$$
\lim _{N \rightarrow \infty} \mathbb{E} \circ \operatorname{tr}\left(\prod_{I \in \sigma} F_{I}\right)=0
$$

This finishes the proof of (4.27), and of the theorem.

\subsection{Calculating $\Lambda_{\pi}$ and certain moments and cumulants}

Here are some results that will allow us to calculate $\Lambda_{\pi}$ for many partitions $\pi$. The first is an easy calculation:

Lemma 4.29. Suppose $n \geq 2$ and $\pi=1_{n}$ is the partition of $\{1, \ldots, n\}$ into one block. Then

$$
\Lambda_{\pi}\left(g_{1}, \ldots, g_{n-1}\right)=\prod_{p=1}^{n-1} \tau\left(g_{p}\right)
$$

is constant.

The next lemma handles the case when $\pi$ splits along two adjacent intervals:

Lemma 4.30. Suppose $\pi=\pi_{1} \cup \pi_{2}$ where, for some $x \in\{1, \ldots, n-1\}, \pi_{1}$ is a partition of $\{1, \ldots, x\}$ and $\pi_{2}$ is a partition of $\{x+1, \ldots, n\}$. Let $\tilde{\pi}_{2} \in \mathcal{P}(n-x)$ be 
the partition obtained from $\pi_{2}$ by shifting $x$ to the left. Then

$$
\Lambda_{\pi}\left(g_{1}, \ldots, g_{n-1}\right)=\Lambda_{\pi_{1}}\left(g_{1}, \ldots, g_{x-1}\right) g_{x} \Lambda_{\tilde{\pi}_{2}}\left(g_{x+1}, \ldots, g_{n-1}\right) .
$$

Proof. Since

$$
\sum_{\{S \in \pi: S \subseteq\{1, \ldots, x\}\}} \sum_{j \in S} s_{j}-s_{j-1}=s_{x}-s_{0}=s_{x}-s_{n}
$$

we see

$$
K_{\pi}^{(t)}=\left\{\left(s_{p}\right)_{p=1}^{n}:\left(s_{p}\right)_{p=1}^{x} \in K_{\pi_{1}}^{(t)},\left(s_{x+p}\right)_{p=1}^{n-x} \in K_{\tilde{\pi}_{2}}^{(t)}\right\}
$$

or, with the obvious identification $K_{\pi}^{(t)}=K_{\pi_{1}}^{(t)} \times K_{\tilde{\pi}_{2}}^{(t)}$, so that the measure $\nu^{(\pi, t)}$ corresponds to the measure $\nu^{\left(\pi_{1}, t\right)} \times \nu^{\left(\tilde{\pi}_{2}, t\right)}$. Thus, we find $F(\pi, t)=F\left(\pi_{1}, t\right) \times F\left(\tilde{\pi}_{2}, t\right)$ and

$$
\begin{aligned}
& \Lambda_{\pi}\left(g_{1}, \ldots, g_{n-1}\right)(t)=\int_{F(\pi, t)}\left(g_{1} \otimes \cdots \otimes g_{n-1} \otimes 1\right) d \nu^{(\pi, t)} \\
& =\int_{F\left(\pi_{1}, t\right)}\left(g_{1} \otimes \cdots \otimes g_{x}\right) d \nu^{\left(\pi_{1}, t\right)} \int_{F\left(\tilde{\pi}_{2}, t\right)}\left(g_{x+1} \otimes \cdots \otimes g_{n-1} \otimes 1\right) d \nu^{\left(\tilde{\pi}_{2}, t\right)} \\
& \quad=\Lambda_{\pi_{1}}\left(g_{1}, \ldots, g_{x-1}\right)(t) g_{x}(t) \Lambda_{\tilde{\pi}_{2}}\left(g_{x+1}, \ldots, g_{n-1}\right)(t) .
\end{aligned}
$$

The next lemma handles a sandwiched interval splitting.

Lemma 4.31. Suppose $\pi \in \mathcal{P}(n)$ and $\pi=\pi_{1} \cup \pi_{2}$, where $\pi_{1}$ is a partition of $S_{1}=$ $\{1, \ldots, x\} \cup\{x+y+1, x+y+2, \ldots, n\}$ and $\pi_{2}$ is a partition of $S_{2}=\{x+1, \ldots, x+y\}$, for some $1 \leq x<x+y \leq n-1$. Let $\tilde{\pi}_{1} \in \mathcal{P}(n-y)$ and $\tilde{\pi}_{2} \in \mathcal{P}(y)$ be the partitions obtained from $\pi_{1}$ by applying the function $L_{1}$ and $\pi_{2}$ by applying the function $L_{2}$, respectively, to all the points in the blocks, where $L_{1}$ and $L_{2}$ are the order-preserving bijections from $S_{1}$ onto $\{1, \ldots, n-y\}$ and from $S_{2}$ onto and $\{1, \ldots, y\}$, respectively. 
Then

$$
\Lambda_{\pi}\left(g_{1}, \ldots, g_{n-1}\right)=\Lambda_{\tilde{\pi}_{1}}\left(g_{1}, \ldots, g_{x-1}, g_{x} \Lambda_{\tilde{\pi}_{2}}\left(g_{x+1}, \ldots, g_{x+y-1}\right) g_{x+y}, g_{x+y+1}, \ldots, g_{n-1}\right)
$$

Proof. Since

$$
\sum_{\{S \in \pi: S \subseteq\{x+1, \ldots, x+y\}\}} \sum_{j \in S} s_{j}-s_{j-1}=s_{x+y}-s_{x}
$$

we see

$$
K_{\pi}=\left\{\left(s_{p}\right)_{p=1}^{n}:\left(s_{1}, \ldots, s_{x}, s_{x+y+1}, \ldots, s_{n}\right) \in K_{\tilde{\pi}_{1}},\left(s_{x+p}\right)_{p=1}^{y} \in K_{\tilde{\pi}_{2}}^{\left(s_{x}\right)}\right\}
$$

and

$$
F(\pi, t)=\left\{\left(s_{p}\right)_{p=1}^{n}:\left(s_{1}, \ldots, s_{x}, s_{x+y+1}, \ldots, s_{n}\right) \in F\left(\tilde{\pi}_{1}, t\right),\left(s_{x+p}\right)_{p=1}^{y} \in F\left(\tilde{\pi}_{2}, s_{x}\right)\right\}
$$

and that the measure $\nu^{(\pi, t)}$ decomposes as

$$
\nu^{(\pi, t)}=\int_{F\left(\tilde{\pi}_{1}, t\right)} \nu^{\left(\tilde{\pi}_{2}, s_{x}\right)} d \nu^{\left(\tilde{\pi}_{1}, t\right)}\left(s_{1}, \ldots, s_{n-y}\right)
$$

Thus, we find

$$
\begin{gathered}
\Lambda_{\pi}\left(g_{1}, \ldots, g_{n-1}\right)(t)=\int_{F(\pi, t)}\left(g_{1} \otimes \cdots \otimes g_{n-1} \otimes 1\right) d \nu^{(\pi, t)} \\
=\int_{F\left(\tilde{\pi}_{1}, t\right)}\left(g_{1} \otimes \cdots \otimes g_{x-1}\right. \\
\otimes g_{x}\left(s_{x}\right)\left(\int_{F\left(\tilde{\pi}_{2}, s_{x}\right)}\left(g_{x+1} \otimes \cdots \otimes g_{x+y-1}\right) \otimes g_{x+y}\left(s_{x}\right) d \nu^{\left(\tilde{\pi}_{2}, s_{x}\right)}\right) \\
\left.\otimes g_{x+y+1} \otimes \cdots \otimes g_{n-1} \otimes 1\right) d \nu^{\left(\tilde{\pi}_{1}, t\right)}\left(s_{1}, \ldots, s_{x}, s_{x+y+1}, \ldots, s_{n-1}\right) \\
=\Lambda_{\tilde{\pi}_{1}}\left(g_{1}, \ldots, g_{x-1}, g_{x} \Lambda_{\tilde{\pi}_{2}}\left(g_{x+1}, \ldots, g_{x+y-1}\right) g_{x+y}, g_{x+y+1}, \ldots, g_{n-1}\right)(t) .
\end{gathered}
$$


The next lemma handles when a partition has two adjacent elements in the same block.

Lemma 4.32. Let $\pi \in \mathcal{P}(n)$ and suppose $\{k, k+1\} \subseteq S \in \pi$ for some $k \in\{1, \ldots, n-$ 1\}. Let $\tilde{\pi} \in \mathcal{P}(n-1)$ be obtained from $\pi$ by gluing $k$ and $k+1$ together; namely, letting

$$
L:\{1, \ldots, n\} \backslash\{k+1\} \rightarrow\{1, \ldots, n-1\}
$$

be the order-preserving bijection, we have

$$
\tilde{\pi}=\{L(S \backslash\{k+1\}): S \in \pi\} .
$$

Then

$$
\Lambda_{\pi}\left(g_{1}, \ldots, g_{n-1}\right)=\Lambda_{\tilde{\pi}}\left(g_{1}, g_{2}, \ldots, \widehat{g_{k}}, \ldots, g_{n-1}\right) \tau\left(g_{k}\right),
$$

where $\widehat{g_{k}}$ indicates that $g_{k}$ has been removed from the list of arguments, while all the others remain.

Proof. In the notation of Remark 4.2, since $k \stackrel{\pi}{\sim} k+1$, we see that in (4.2) the condition for $\left(s_{p}\right)_{p=1}^{n}$ to belong to $K_{\pi}$ involves $s_{k}$ only in the summation over the block of $\pi$ that contains $k$, and in this summation $s_{k}$ cancels with $-s_{k}$. Thus, we easily see

$$
K_{\pi}=\left\{\left(s_{p}\right)_{p=1}^{n}:\left(s_{1}, \ldots, \widehat{s_{k}}, \ldots, s_{n}\right) \in K_{\tilde{\pi}}\right\}
$$

and, under the corresponding identification $K_{\pi}=K_{\tilde{\pi}} \times \mathbf{R}$, for every $t \in[0,1]$ we have the identification of measures

$$
\nu^{(\pi, t)}=\nu^{(\tilde{\pi}, t)} \times \lambda,
$$


where $\lambda$ is Lebesgue measure on $\mathbf{R}$. Using (4.3), we get

$$
\begin{aligned}
\Lambda_{\pi}\left(g_{1}, \ldots, g_{n-1}\right)(t) & =\int_{F(\pi, t)}\left(g_{1} \otimes \cdots \otimes g_{n-1} \otimes 1\right) d \nu^{(\pi, t)} \\
& =\int_{F(\tilde{\pi}, t)}\left(g_{1} \otimes \cdots \otimes \widehat{g_{k}} \otimes \cdots \otimes g_{n-1} \otimes 1\right) d \nu^{(\tilde{\pi}, t)} \int_{[0,1]} g_{k} d \lambda \\
& =\Lambda_{\tilde{\pi}}\left(g_{1}, g_{2}, \ldots, \widehat{g_{k}}, \ldots, g_{n-1}\right)(t) \tau\left(g_{k}\right) .
\end{aligned}
$$

Lemma 4.33. Let $n \geq 2$ and suppose $\pi \in \mathcal{P}(n)$ has 1 and $n$ in the same block. Then for all $g_{1}, \ldots, g_{n-1} \in C[0,1], \Lambda_{\pi}\left(g_{1}, g_{2}, \ldots, g_{n-1}\right)$ is a constant function. Moreover, letting $\tilde{\pi} \in \mathcal{P}(n-1)$ be the restriction of $\pi$ to $\{1, \ldots, n-1\}$, we have

$$
\Lambda_{\pi}\left(g_{1}, \ldots, g_{n-1}\right)=\tau\left(\Lambda_{\tilde{\pi}}\left(g_{1}, \ldots, g_{n-2}\right) g_{n-1}\right)
$$

Proof. This is much like the proof of Lemma 4.32. Recalling that we use the convention $s_{0}=s_{n}$, since $1 \stackrel{\pi}{\sim} n$, in $(4.2)$ the condition for $\left(s_{p}\right)_{p=1}^{n}$ to belong to $K_{\pi}$ involves $s_{n}$ only in the summation over the block of $\pi$ that contains $n$, and in this summation $s_{n}$ cancels with $-s_{0}$. Thus, we have

$$
K_{\pi}=\left\{\left(s_{p}\right)_{p=1}^{n}:\left(s_{1}, \ldots, s_{n-1}\right) \in K_{\tilde{\pi}}\right\}
$$

Moreover, $F(\pi, t)=\left(K_{\tilde{\pi}} \cap[0,1]^{n-1}\right) \times\{t\}$ and $\nu^{(\pi, t)}$ corresponds to simply $(n-|\pi|)$ - 
dimensional Lebesgue measure on $K_{\tilde{\pi}}$. Using (4.3), we get

$$
\begin{aligned}
\Lambda_{\pi}\left(g_{1}, \ldots, g_{n-1}\right)(t) & =\int_{F(\pi, t)}\left(g_{1} \otimes \cdots \otimes g_{n-1} \otimes 1\right) d \nu^{(\pi, t)} \\
& =\int_{[0,1]}\left(\int_{F(\tilde{\pi}, s)}\left(g_{1} \otimes \cdots \otimes g_{n-1}\right) d \nu^{(\tilde{\pi}, s)}\right) d \lambda(s) \\
& =\int_{[0,1]} \Lambda_{\tilde{\pi}}\left(g_{1}, g_{2}, \ldots, g_{n-2}\right)(s) g_{n-1}(s) d \lambda(s) \\
& =\tau\left(\Lambda_{\tilde{\pi}}\left(g_{1}, g_{2}, \ldots, g_{n-2}\right) g_{n-1}\right) .
\end{aligned}
$$

\section{Lemma 4.34.}

$$
\tau\left(\Lambda_{\pi}\left(g_{1}, \ldots, g_{n-1}\right) g_{n}\right)=\tau\left(\Lambda_{L(\pi)}\left(g_{2}, \ldots, g_{n}\right) g_{1}\right)
$$

where $L(\pi) \in \mathcal{P}(n)$ is obtained from $\pi$ by left rotating the underlying set $\{1, \ldots, n\}$.

Proof. Examining Remark 4.2, we see

$$
\tau\left(\Lambda_{\pi}\left(g_{1}, \ldots, g_{n-1}\right) g_{n}\right)=\int_{K_{\pi} \cap[0,1]^{n}} g_{1} \otimes \cdots \otimes g_{n} d \nu^{(\pi)}
$$

where $\nu^{(\pi)}$ is the $(n-|\pi|)$-dimensional Lebesgue measure on $K_{\pi}$ corresponding to the restriction to $K_{\pi}$ of the Euclidean metric on $\mathbf{R}^{n}$. Moreover, we find

$$
K_{L(\pi)}=\left\{\left(s_{2}, \ldots, s_{n}, s_{1}\right):\left(s_{p}\right)_{p=1}^{n} \in K_{\pi}\right\}
$$

and that under this identification, $d \nu^{(L(\pi))}\left(s_{2}, \ldots, s_{n}, s_{1}\right)$ corresponds to 
$d \nu^{(\pi)}\left(s_{1}, \ldots, s_{n}\right)$. Thus, we get

$\tau\left(\Lambda_{\pi}\left(g_{1}, \ldots, g_{n-1}\right) g_{n}\right)=\int_{K_{L(\pi)} \cap[0,1]^{n}} g_{2} \otimes \cdots \otimes g_{n} \otimes g_{1} d \nu^{(L(\pi))}=\tau\left(\Lambda_{L(\pi)}\left(g_{2}, \ldots, g_{n}\right) g_{1}\right)$

The following is easily checked:

Lemma 4.35. Suppose $\pi$ is any partition of $\{1, \ldots, n\}$. Then

$$
\Gamma_{\pi}(1, \ldots, 1)=1
$$

Here is an immediate consequence of the above fact and (4.24).

Proposition 4.36. For any $n \in \mathbf{N}$ and any $b_{1}, \ldots, b_{n} \in B$,

$$
\mathcal{E}\left(X b_{1} X^{*} X b_{2} X^{*} \cdots X b_{n} X^{*}\right) \in \mathbf{C} 1
$$

From Lemmas 4.29-4.33, we easily get the following:

Lemma 4.37. Suppose $\pi$ is a noncrossing partition of $\{1, \ldots, n\}$. Then

$$
\Lambda_{\pi}(1,1, \ldots, 1)=1
$$

Since all partitions of $\{1,2,3\}$ are noncrossing, from (4.23)-(4.24) we easily get:

$$
\begin{aligned}
\mathcal{E}\left(X X^{*}\right) & =\mathcal{E}\left(X^{*} X\right)=1, \\
\mathcal{E}\left(\left(X X^{*}\right)^{2}\right) & =\mathcal{E}\left(\left(X^{*} X\right)^{2}\right)=2, \\
\mathcal{E}\left(\left(X X^{*}\right)^{3}\right) & =\mathcal{E}\left(\left(X^{*} X\right)^{3}\right)=5 .
\end{aligned}
$$


There are 14 noncrossing partitions of $\{1,2,3,4\}$ and one crossing partition, namely, $\pi_{4}=\{\{1,3\},\{2,4\}\}$. Using Remark 4.2, we calculate

$$
\begin{aligned}
\Lambda_{\pi_{4}}\left(b_{1}, b_{2}, b_{3}\right)(t)=\int_{0}^{t} b_{1}\left(s_{1}\right) & \int_{0}^{1-t+s_{1}} b_{2}\left(s_{2}\right) b_{3}\left(t-s_{1}+s_{2}\right) d s_{2} d s_{1} \\
& +\int_{t}^{1} b_{1}\left(s_{1}\right) \int_{s_{1}-t}^{1} b_{2}\left(s_{2}\right) b_{3}\left(t-s_{1}+s_{2}\right) d s_{2} d s_{1} .
\end{aligned}
$$

From (4.34), we calculate

$$
\Lambda_{\pi_{4}}(1,1,1)(t)=\frac{1}{2}+t(1-t)
$$

Consequently, from (4.23) and (4.24), we find

$$
\begin{aligned}
\mathcal{E}\left(\left(X X^{*}\right)^{4}\right) & =14+\frac{2}{3} \\
\mathcal{E}\left(\left(X^{*} X\right)^{4}\right)(t) & =14+\frac{1}{2}+t(1-t) .
\end{aligned}
$$

Unlike with scalar-valued R-diagonality in the tracial setting, in the $B$-valued case, *-freeness is not guaranteed in a polar decomposition. This phenomenon was seen in [7], but is also exhibited by the asymptotic limit of the random Vandermonde matrices:

Proposition 4.38. The element $X$ does not have the same *-distribution as any element in a B-valued *-noncommutative probability space of the form $P U$, with $U$ unitary, $P \geq 0$ and such that $U$ and $P$ are $*$-free over $B$.

Proof. Suppose for contradiction such a realization $X \sim P U$ is possible for $P$ and $U$ in a $B$-valued $*$-noncommutative probability space $(\widetilde{A}, \widetilde{\mathcal{E}})$. 
From (4.35)-(4.36), we have

$$
\begin{aligned}
\widetilde{\mathcal{E}}\left(P^{8}\right) & =14+\frac{2}{3} \\
\widetilde{\mathcal{E}}\left(U^{*} P^{8} U\right)(t) & =14+\frac{1}{2}+t(1-t) .
\end{aligned}
$$

However, by $*$-freeness, we calculate

$$
\widetilde{\mathcal{E}}\left(U^{*} P^{8} U\right)=\widetilde{\mathcal{E}}\left(U^{*} \widetilde{\mathcal{E}}\left(P^{8}\right) U\right)=\widetilde{\mathcal{E}}\left(U^{*}\left(14+\frac{2}{3}\right) U\right)=14+\frac{2}{3}
$$

which is a contradiction.

Question 4.4.1. Can $X$ have the same $*$-distribution as a product $U P$ for some $U$ and $P$ as described in Proposition 4.38?

The next result answers negatively a question of G. Tucci.

Proposition 4.39. With respect to the trace $\tau \circ \mathcal{E}, X$ is not a scalar-valued $R$ diagonal element.

Proof. If it were scalar-valued R-diagonal, then we would have

$$
\tau \circ \mathcal{E}\left(\left(\left(X^{*} X\right)^{4}-\frac{44}{3}\right)\left(\left(X X^{*}\right)^{2}-2\right)\left(\left(X^{*} X\right)^{4}-\frac{44}{3}\right)\left(\left(X X^{*}\right)^{2}-2\right)\right)=0 .
$$

Letting $b \in B$ be $b(t)=14+\frac{1}{2}+t(1-t)$, by $B$-valued R-diagonality of $X$ and (4.35) and (4.32), we have

$$
\mathcal{E}\left(\left(\left(X^{*} X\right)^{4}-b\right)\left(\left(X X^{*}\right)^{2}-2\right)\left(\left(X^{*} X\right)^{4}-b\right)\left(\left(X X^{*}\right)^{2}-2\right)\right)=0
$$

Writing $\left(X^{*} X\right)^{4}-\frac{44}{3}=\left(\left(X^{*} X\right)^{4}-b\right)+\left(b-\frac{44}{3}\right)$, expanding, distributing, using (4.38), 
$B$-valued R-diagonality again and (4.33), we get

$$
\begin{aligned}
\mathcal{E}\left(\left(\left(X^{*} X\right)^{4}-\frac{44}{3}\right)\right. & \left.\left(\left(X X^{*}\right)^{2}-2\right)\left(\left(X^{*} X\right)^{4}-\frac{44}{3}\right)\left(\left(X X^{*}\right)^{2}-2\right)\right) \\
& =\mathcal{E}\left(\left(b-\frac{44}{3}\right)\left(\left(X X^{*}\right)^{2}-2\right)\left(b-\frac{44}{3}\right)\left(\left(X X^{*}\right)^{2}-2\right)\right) \\
& =\left(b-\frac{44}{3}\right)\left(\mathcal{E}\left(\left(X X^{*}\right)^{2}\left(b-\frac{44}{3}\right)\left(X X^{*}\right)^{2}\right)-4\left(b-\frac{44}{3}\right)\right)
\end{aligned}
$$

Using (4.24) we find that for $b^{\prime} \in B$,

$$
\mathcal{E}\left(\left(X X^{*}\right)^{2} b^{\prime}\left(X X^{*}\right)^{2}\right)=10 \tau\left(b^{\prime}\right)+\left(4+\frac{2}{3}\right) b^{\prime},
$$

and thus the quantity $(4.39)$ equals $\frac{2}{3}\left(b-\frac{44}{3}\right)^{2}$. But

$$
\frac{2}{3} \tau\left(\left(b-\frac{44}{3}\right)^{2}\right)=\frac{2}{3} \int_{0}^{1}\left(\left(t(1-t)-\frac{1}{6}\right)^{2} d t=\frac{1}{270} \neq 0\right.
$$

which shows that (4.37) fails to hold. 


\section{SUMMARY}

In this section, we summarize results proven in the dissertation.

\subsection{Mean ergodic convergence}

Theorem. Let $X$ be a Banach space having the bounded compact approximation property. Let $T$ be an operator on $X$. Let $\dot{T}$ be the image of $T$ in the Calkin algebra.

Then $\frac{\dot{I}+\dot{T}+\ldots+\dot{T}^{n}}{n}$ converges in norm if and only if $\frac{\left\|\dot{T}^{n}\right\|}{n} \rightarrow 0$ and there is an essentially maximal subspace of $X$ on which $I-T$ is compact.

\subsection{Universal operators}

Theorem. Suppose that $\mathcal{C}$ is a collection of uniformly bounded operators in $\mathcal{B}(\mathcal{H})$ with disjoint spectra. Then the following statements are equivalent.

(i) There exists an operator $T \in \mathcal{B}(\mathcal{H})$ such that every operator in $\mathcal{C}$ is compalent to a reducing part of $T$.

(ii) Every operator in $\mathcal{C}$ outside a countable subset is quasidiagonal.

\subsection{Approximate similarity}

Theorem. Suppose that $T_{1}, T_{2} \in \mathcal{B}(\mathcal{H})$. Then $T_{1}$ and $T_{2}$ are approximately similar if and only if $T_{1}$ is approximately unitarily equivalent to an operator $T_{1}^{\prime}$ that is similar to an operator $T_{2}^{\prime}$ that is apprxoimately unitarily equivalent to $T_{2}$.

\subsection{Unitary orbits}

Theorem. Let $T \in \mathcal{B}(\mathcal{H})$. Then the strong, weak and $*$-strong closures of $T$ are given by the set of all restrictions, compressions and reducing parts of operators that are approximately unitarily equivalent to $T$, respectively. 


\subsection{Random Vandermonde matrices}

Theorem. Let $X_{N}$ be the $N \times N$ random Vandermonde matrix whose $(i, j)$-th entry is $N^{-1 / 2} \zeta_{i}^{j}$, where $\zeta_{1}, \ldots, \zeta_{N}$ are independent with uniform distribution on the unit circle. Then $X_{N}$ is not asymptotically $R$-diagonal but it is asymptotically $R$-diagonal with amalgamation over the deterministic diagonals

$$
\operatorname{diag}\left(b\left(\frac{1}{N}\right), b\left(\frac{2}{N}\right), \ldots, b\left(\frac{N}{N}\right)\right), \quad b \in C[0,1]
$$




\section{REFERENCES}

[1] N. I. Akhiezer, The classical moment problem and some related questions in analysis, Translated by N. Kemmer, Hafner Publishing Co., New York, 1965.

[2] R. Bhatia, Matrix Analysis, Graduate Texts in Mathematics, vol. 169, SpringerVerlag, New York, 1997.

[3] E. Bishop, Spectral theory for operators on a Banach space, Trans. Amer. Math. Soc. 86 (1957) 414-445.

[4] M. T. Boedihardjo and W. B. Johnson, On mean ergodic convergence in the Calkin algebras, Proc. Amer. Math. Soc. 143 (2015), no. 6, 2451-2457.

[5] M. T. Boedihardjo, A coordinate free characterization of certain quasidiagonal operators, Indiana Univ. Math. J. 64 (2015), no. 2, 515-531.

[6] M. Boedihardjo and K. Dykema, Asymptotic *-moments of some random Vandermonde matrices, http://arxiv.org/abs/1602.02815, 8 Feb 2016.

[7] M. Boedihardjo and K. Dykema, Algebra-valued R-diagonal elements, http://arxiv.org/abs/1512.06321, 12 Jan 2016.

[8] J. W. Calkin, Two-sided ideals and congruences in the ring of bounded operators in Hilbert space, Ann. of Math. 42 (1941), 839-873.

[9] J. B. Conway and D. W. Hadwin, Strong limits of normal operators, Glasgow Math. J. 24 (1983), no. 1, 93-96.

[10] K. R. Davidson, Estimating the distance between unitary orbits, J. Operator Theory 20 (1988), 21-40. 
[11] J. Diestel, H. Jarchow and A. Tonge, Absolutely Summing Operators, Cambridge University Press, Cambridge, 1995.

[12] R. G. Douglas, Banach Algebra Techniques in Operator Theory, Springer-Verlag, New York, 1998.

[13] N. Dunford, Spectral theory. I. Convergence to projections, Trans. Amer. Math. Soc. 54 (1943), 185-217.

[14] I. S. Edelstein and P. Wojtaszczyk, On projections and unconditional bases in direct sums of Banach spaces, Studia Math. 56 (1976), 263-276.

[15] C. Foiaş, C. Pasnicu and D. Voiculescu, Weak limits of almost invariant projections, J. Operator Theory 2 (1979) 79-93.

[16] G. Godefroy and P. Saphar, Duality in spaces of operators and smooth norms on Banach spaces, Illinois J. Math. 32 (1988), no. 4, 672-695.

[17] I. Gohberg, S. Goldberg and A. M. Kaashoek, Classes of linear operators: Vol. I, Birkäuser, Basel, 1990.

[18] D. Hadwin, An operator-valued spectrum, Indiana Univ. Math. J. 26 (1977) 329-340.

[19] D. Hadwin, Completely positive maps and approximate equivalence, Indiana Univ. Math. J. 36 (1987) 211-228.

[20] D. Hadwin, A note on approximate similarity, J. Korean Math. Soc. 38 (2001) 1157-1166.

[21] P. R. Halmos, Ten problems in Hilbert space, Bull. Amer. Math. Soc. 76 (1970) 887-933. 
[22] D. A. Herrero, Quasidiagonality, similarity and approximation by nilpotent operators, Indiana Univ. Math. J. 30 (1981) 199-233.

[23] W. B. Johnson and A. Szankowski, Complementably universal Banach spaces, Studia Math. 58 (1976) 91-97.

[24] M. Lin, On the uniform ergodic theorem, Proc. Amer. Math. Soc. 43 (1974) 337-340.

[25] J. Lindenstrauss, Extension of compact operators, Mem. Amer. Math. Soc. No. 48 (1964) $112 \mathrm{pp}$.

[26] J. Lindenstrauss and L. Tzafriri, Classical Banach Spaces I, Springer-Verlag, Berlin, 1977, Sequence spaces.

[27] E. R. Lorch, Means of iterated transformations in reflexive vector spaces, Bull. Amer. Math. Soc. 45 (1939), 945-947.

[28] M. Mbekhta and J. Zemánek, Sur le theoreme ergodique uniforme et le spectre, C. R. Acad. Sci. Paris Sér. I Math. 317 (1993), 1155-1158.

[29] A. Nica and R. Speicher, R-diagonal pairs - a common approach to Haar unitaries and circular elements, Free Probability Theory (Waterloo, Ontario), Fields Inst. Commun., vol. 12, Amer. Math. Soc.,1997, pp. 149-188.

[30] Ø. Ryan and M. Debbah, Asymptotic behavior of random Vandermonde matrices with entries on the unit circle, IEEE Trans. Inform. Theory 55 (2009), 3115-3147.

[31] Ø. Ryan and M. Debbah, Convolution operations arising from Vandermonde matrices, IEEE Trans. Inform. Theory 57 (2011) 4647-4659. 
[32] P. Śniady and R. Speciher, Continuous family of invariant subspaces for $R$ diagonal operators, Invent. Math. 146 (2001), 329-363.

[33] G. H. Tucci (2012), private communication.

[34] G. H. Tucci and P. A. Whiting, Eigenvalue results for large scale random Vandermonde matrices with unit complex entries, IEEE Trans. Inform. Theory 57 (2011), 3938-3954.

[35] G. H. Tucci and P. A. Whiting, Asymptotic behavior of the maximum and minimum singular value of random Vandermonde matrices, J. Theoret. Probab. 27 (2014), no. 3, 826-862.

[36] C. M. Pearcy, Some Recent Developments in Operator Theory, CBMS Reg. Conf. Ser. Math. 36, Amer. Math. Soc., Providence, 1978.

[37] H. Radjavi and P. Rosenthal, Invariant Subspaces, second edition, Dover Publications, New York, 2003.

[38] G. A. Reid, On the Calkin representations, Proc. London Math. Soc. 23 (1971) $547-564$.

[39] F. Riesz, B. Sz.-Nagy, Functional Analysis, Dover, New York, 1990.

[40] M. Rosenblum, On the operator equation $B X-X A=Q$, Duke Math. J. 23 (1956), 263-269.

[41] D. Voiculescu, A non-commutative Weyl-von Neumann theorem, Rev. Roumaine Math. Pures Appl. 21 (1976), no. 1, 97-113. 